\title{
2008 Florida Plant Disease Management Guide: Chemical Control Guide for Diseases of Vegetables, Revision No. 191
}

Ken Pernezny, Amanda Gevens, Tim Momol, Aaron Palmateer, Natalia Peres, Richard Raid, Pam Roberts, Gary Vallad, and Shousan Zhang ${ }^{2}$

This publication is a guide to lawful use of sprayable chemicals intended for control of plant diseases affecting vegetables grown in Florida.

Control of plant diseases is achieved by utilizing numerous cultural, biological, regulatory and chemical tactics. The performance of a chemical can be enhanced through various non chemical tactics to reduce inocula. Additionally, within chemical control, numerous variables can influence the performance of a chemical. Some examples of variables affecting success of chemical-application technology include the following: time of applications, nozzle arrangement, spray dilution, chemical rate per unit area, adjuvants, and rainfall. Beginning spray programs prior to or at first sign of disease is best.
USE A SPRAY ADJUVANT WITH A SPREADER AND A STICKER IN CONJUNCTION WITH SOME WETTABLE POWDER AND SOME FLOWABLE FORMULATIONS. SPREADER-STICKER ADJUVANTS ARE USEFUL, PARTICULARLY ON SLICK LEAF AND VERTICALLY LEAF-ORIENTED CROPS, SUCH AS ONIONS, CRUCIFERS AND SWEET CORN. DO NOT USE SPRAY ADJUVANTS IF THE LABEL INDICATES THAT ADJUVANTS SHOULD NOT BE USED. FOR EXAMPLE, SEVERAL LABELS WARN AGAINST USE OF SILICON ADJUVANTS WITH THOSE PRODUCTS.

The listing that follows does not include all legally available chemicals sold in Florida for the purpose of plant-disease control. For a

1. This document is PPP6, one of a series of the Department of Plant Pathology, Florida Cooperative Extension Service, Institute of Food and Agricultural Sciences, University of Florida. Original publicatin date, 2003. Revised, September 2008. Visit the EDIS Web site at http://edis.ifas.ufl.edu. This document is also known as Extension Plant Pathology Report No. 6 and as Plant Protection Pointer No. 6.

2. Ken Pernezny, professor, Plant Pathology Department, Everglades Research and Education Center--Belle Glade, FL; Amanda Gevens, assistant professor, Plant Pathology Department; Tim Momol, professor, Plant Pathology Department, and extension director, Central Florida District; Aaron Palmateer, assistant professor, Plant Pathology Department, Tropical Reserach and Education Center--Homestead, FL; Natalia Peres, assistant professor, Plant Pathology Department, Gulf Coast Research and Education Center--Wimauma, FL; Richard Raid, professor, Plant Pathology Department, Gulf Coast Research and Education Center--Wimauma, FL; Pam Roberts, assistant professor, Plant Pathology Department, Southwest Florida Research and Education Center--Immokalee, FL; and Shousan Zhang, assistant professor, Plant Pathology Department, Tropical Research and Education Center--Homestead, FL, Florida Cooperative Extension Service, Institute of Food and Agricultural Sciences, University of Florida, Gainesville, FL 32611. The use of trade names in this publication is solely for the purpose of providing specific information. UF/IFAS does not guarantee or warranty the products named, and references to them in this publication does not signify our approval to the exclusion of other products of suitable composition.

The Institute of Food and Agricultural Sciences (IFAS) is an Equal Opportunity Institution authorized to provide research, educational information and other services only to individuals and institutions that function with non-discrimination with respect to race, creed, color, religion, age, disability, sex, sexual orientation, marital status, national origin, political opinions or affiliations. U.S. Department of Agriculture, Cooperative Extension Service, University of Florida, IFAS, Florida A. \& M. University Cooperative Extension Program, and Boards of County Commissioners Cooperating. Interim Dean Millie Ferrer. 
comprehensive list, contact the Florida Department of Agriculture and Consumer Services (FDACS), Division of of Agricultural Services, Bureau of Pesticides - http://www.flaes.org/Pesticide/.

Chemicals listed in this guide are the following:

1) those for which data from the State of Florida are available for the active ingredient;

2) chemicals for which no other compound(s) is (are) known to be available, but use is lawful according to the United States Environmental Protection Agency (EPA);

3) chemicals that are legal to use and lack data from FDACS, but would be expected to perform satisfactorily based upon professional judgement.

It may seem rhetorical, but read the label; you paid for it. Read labels for information about crop-rotational limitations with fungicides. Also note within this publication the crop groupings for tolerance establishment. Keep in mind, too, that REENTRY into fields treated with any pesticide is restricted by the use of a time interval, as well as by type of clothing worn, and necessary protective wear. For example, labels of products containing chlorothalonil state a 12-hour reentry interval. However, if adequate eye protection is not used, the reentry interval is seven days. Label information supersedes all other sources of information. If, for example, the label does not prohibit greenhouse use, the material can be used in the greenhouse.

\section{Rotate unrelated chemicals in your spray} program for resistance management. Continued use of the same or related chemicals can result in insensitivity (resistance) of the target pest to that chemical or to other chemicals in the same group. For example, all strobilurin fungicides are in Group 11 as indicated on the fungicide labels. Group 11 fungicides include the following: Flint, Quadris, Quardris Opti, Amistar, Headline, Cabrio, and Pristine. 


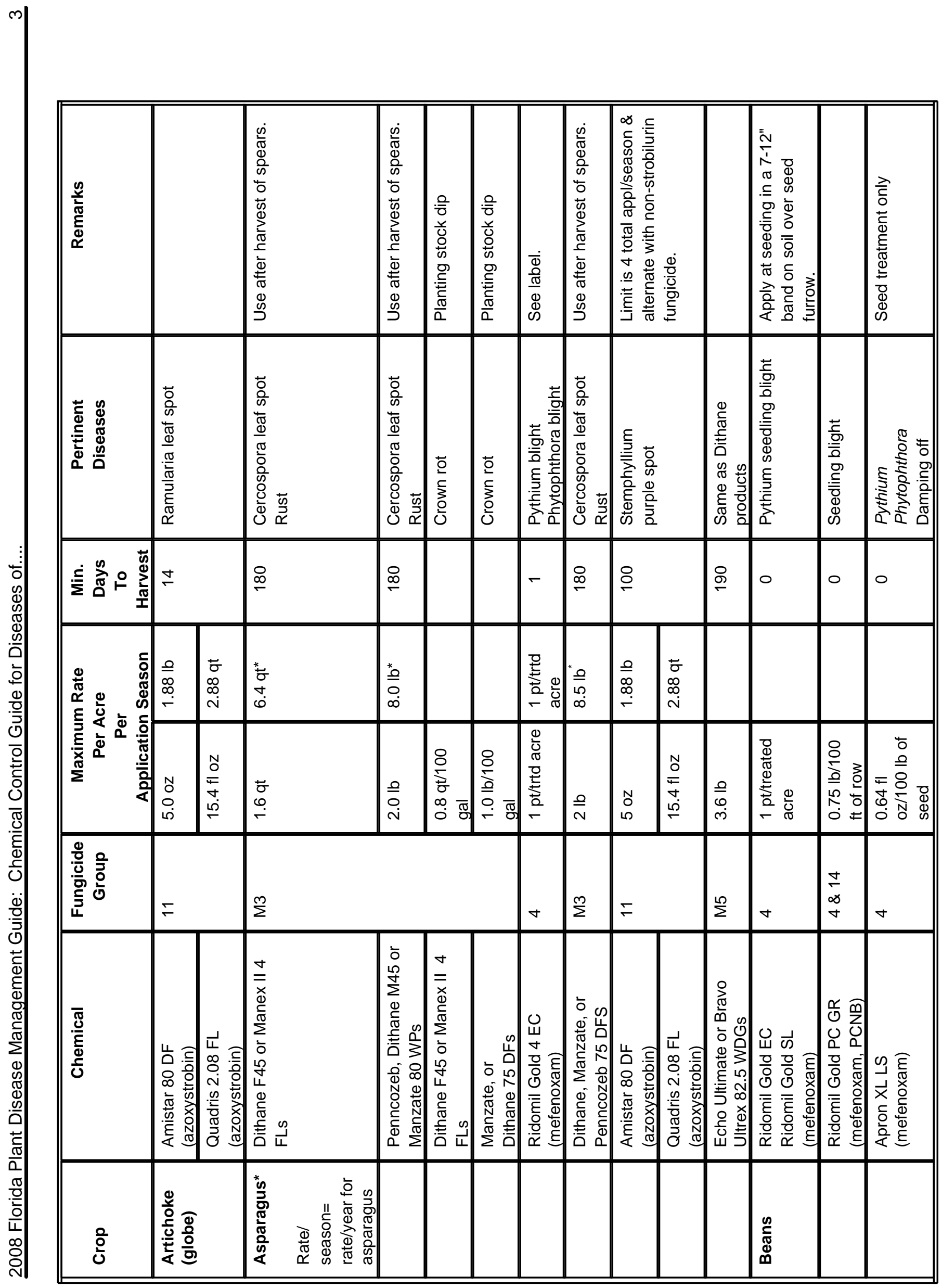




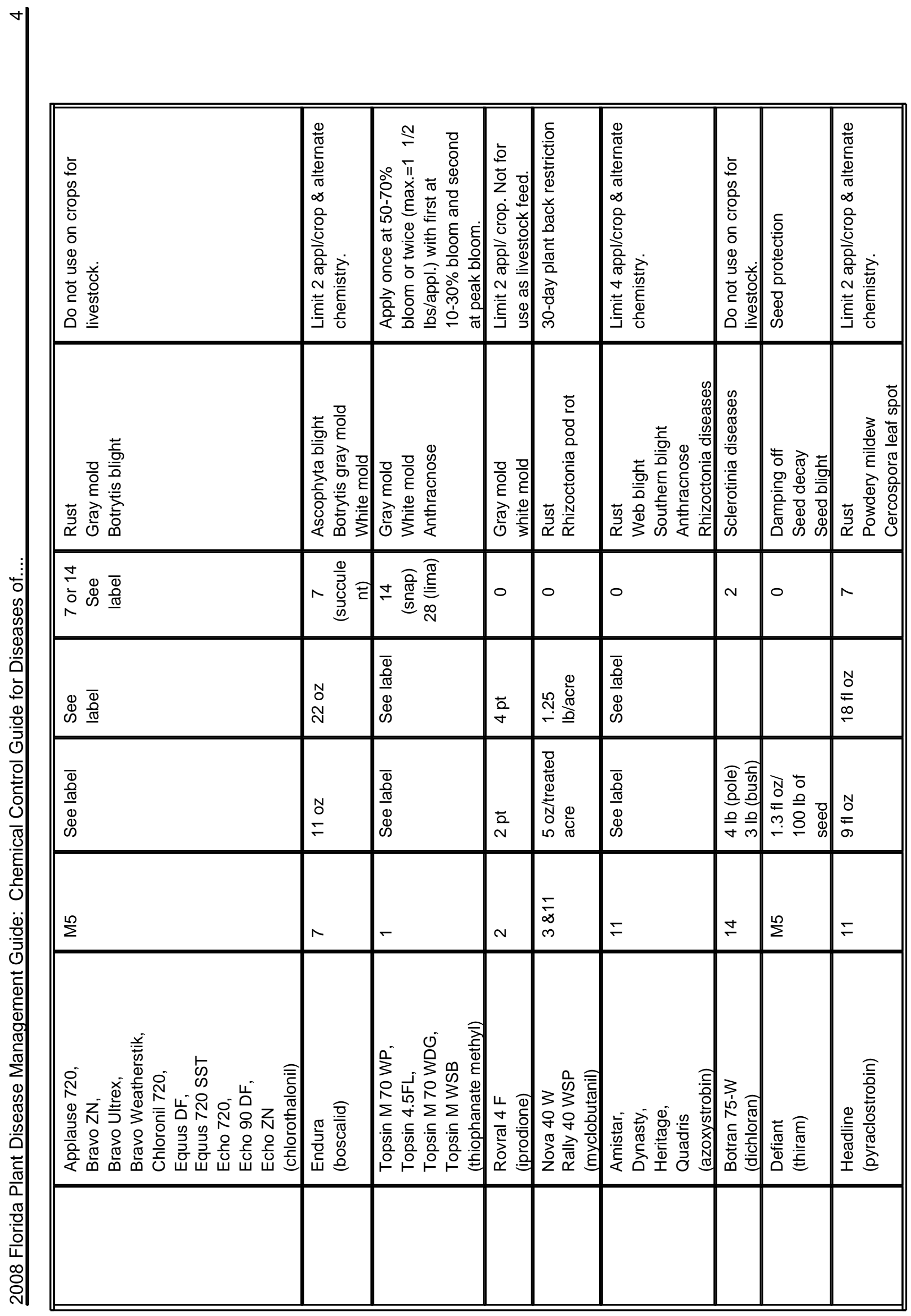




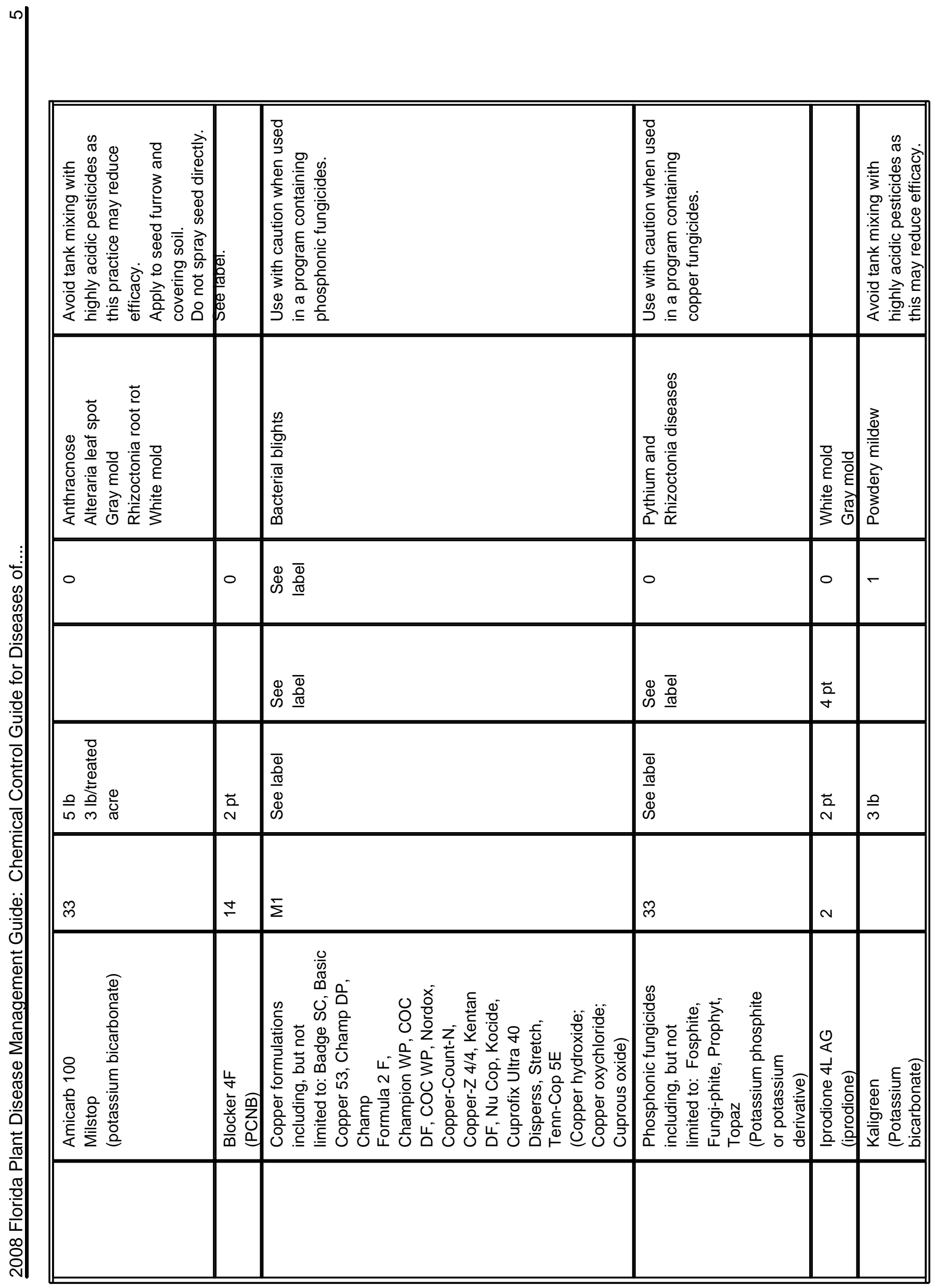




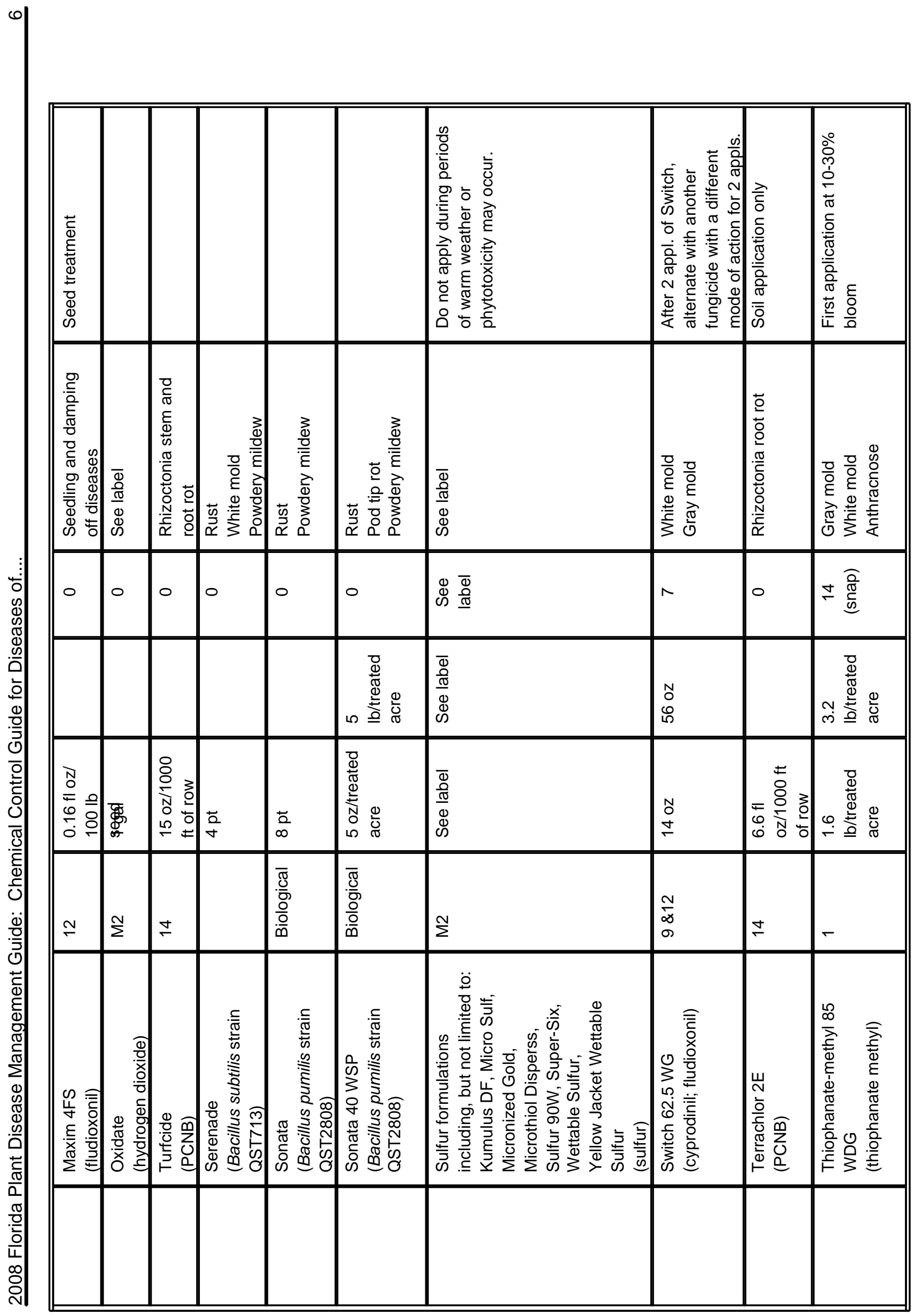




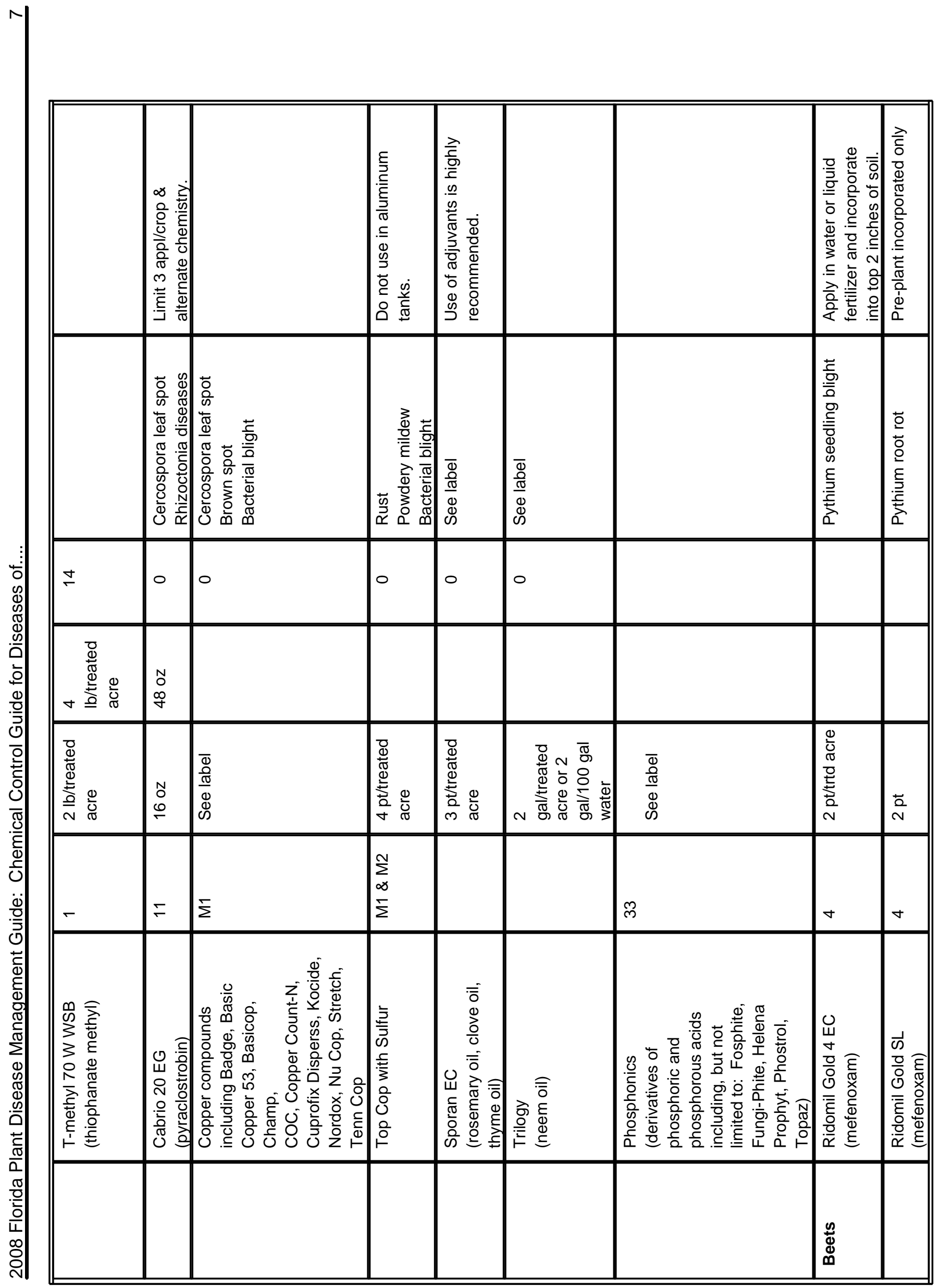




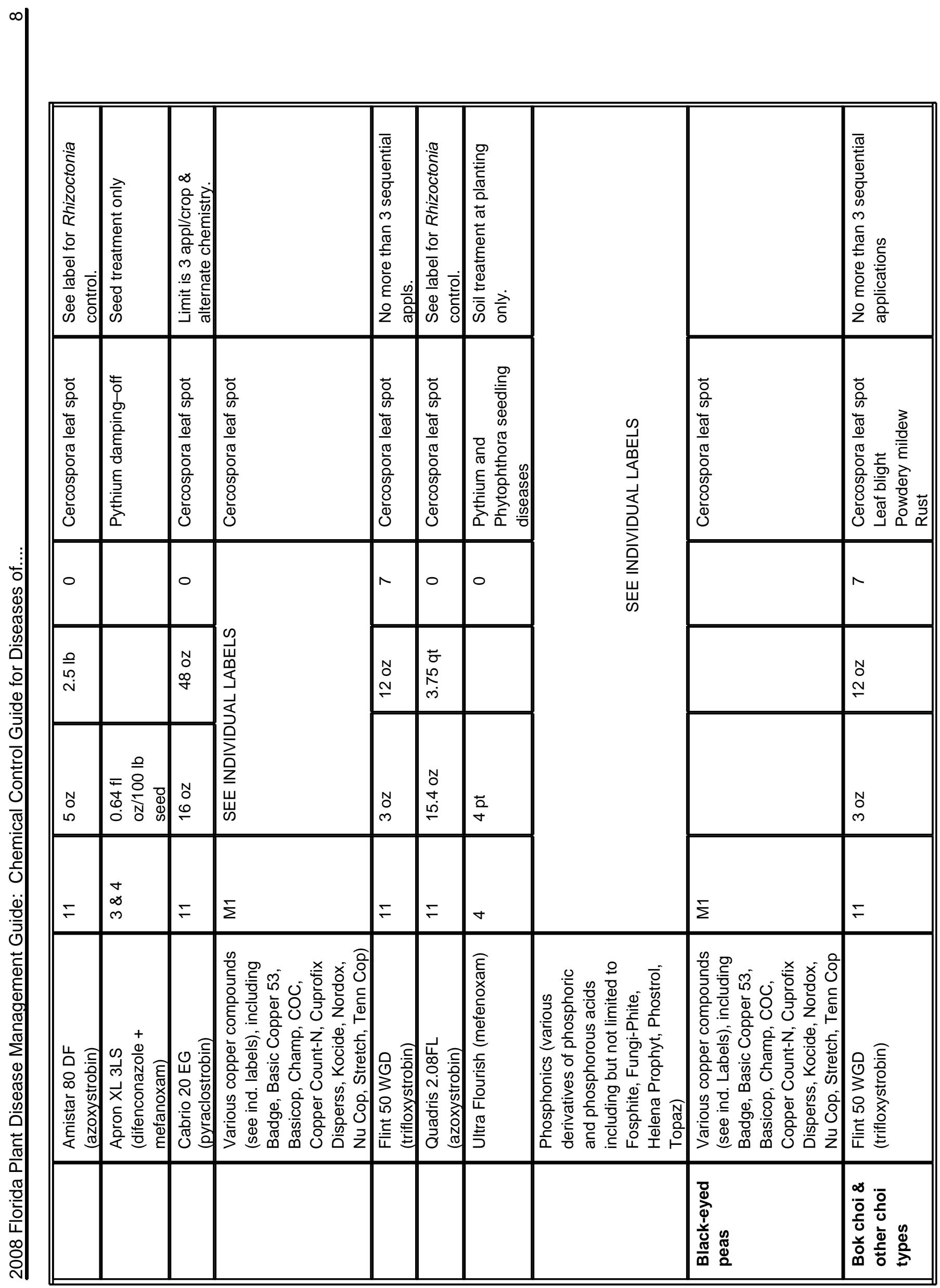




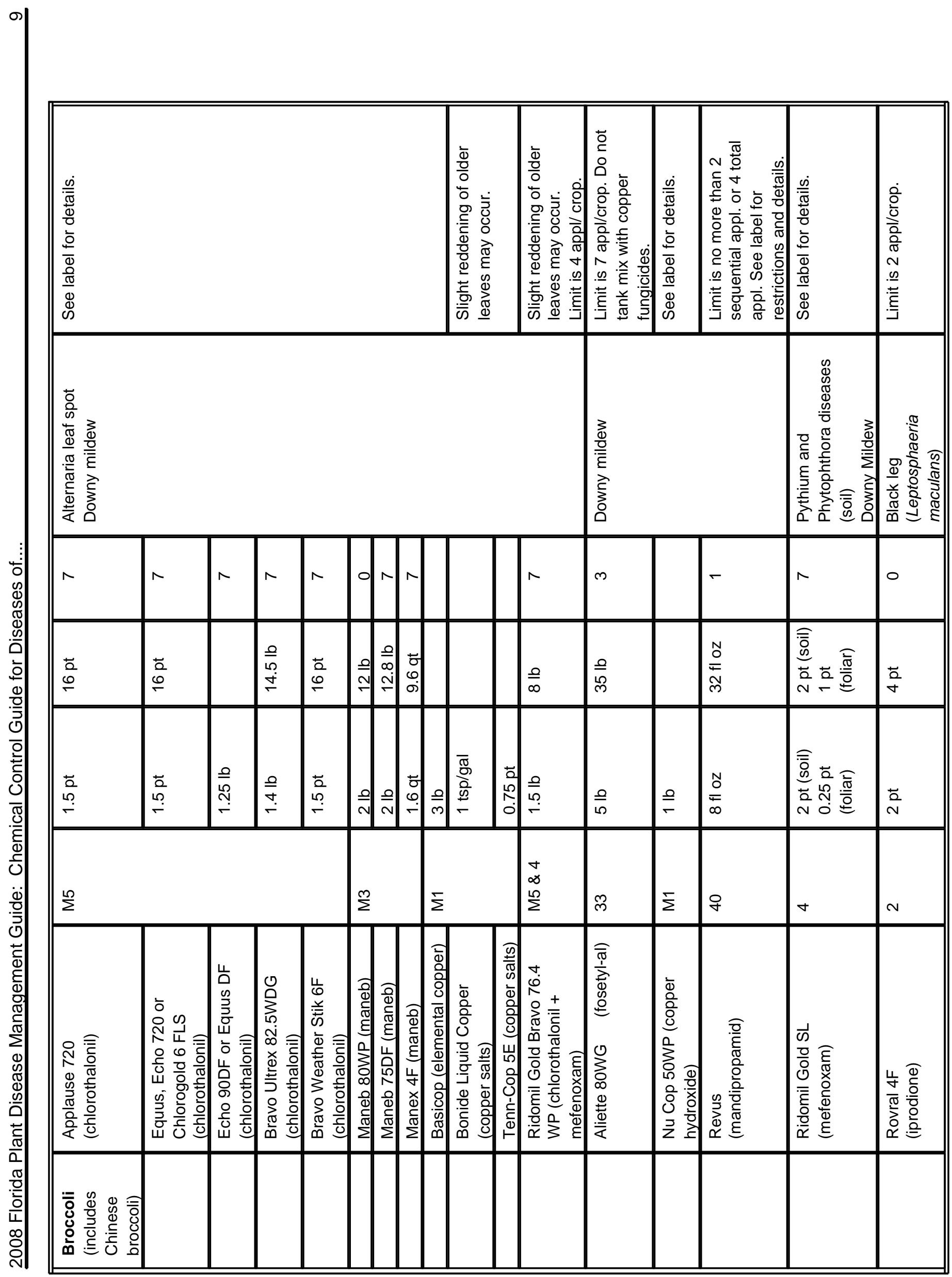




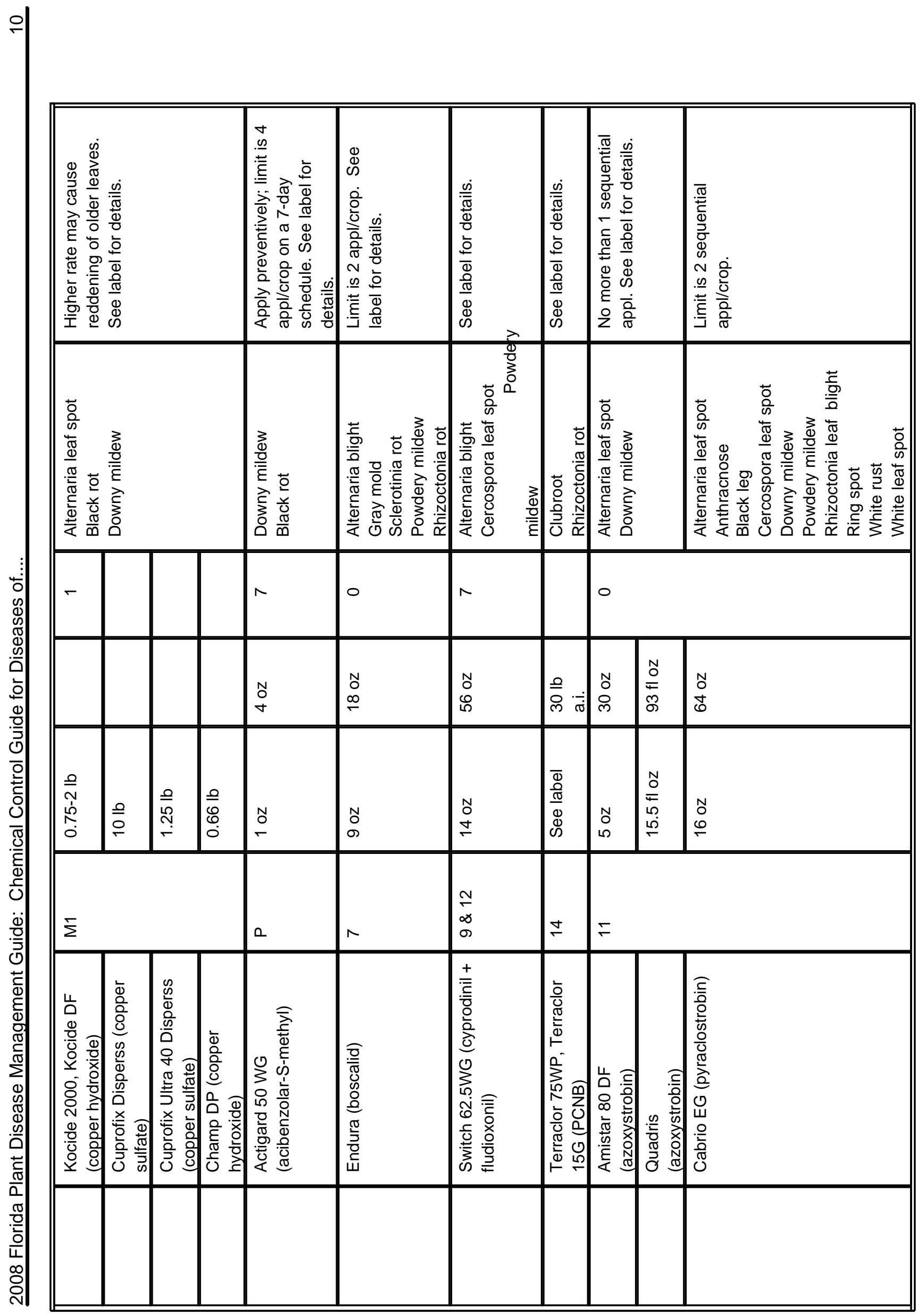




\begin{tabular}{|c|c|c|c|c|c|c|c|c|}
\hline 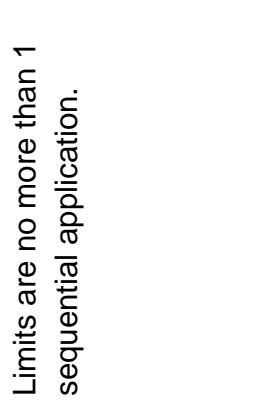 & 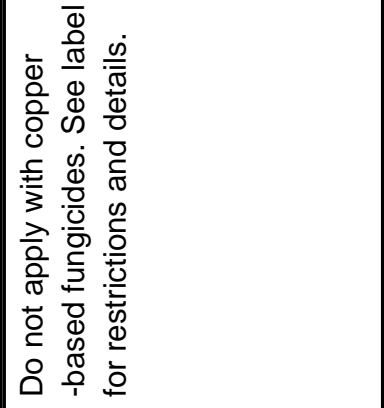 & 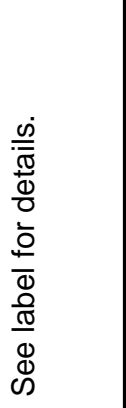 & 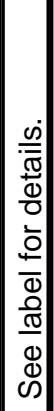 & 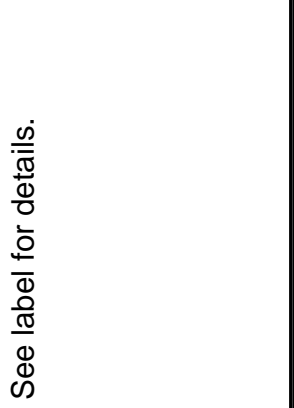 & $\begin{array}{l}\frac{\infty}{0} \\
\overline{\bar{\pi}} \\
\frac{0}{0} \\
\dot{0} \\
\frac{0}{0} \\
\frac{0}{\sigma} \\
\frac{\pi}{0} \\
\infty \\
\infty\end{array}$ & \multicolumn{3}{|l|}{ 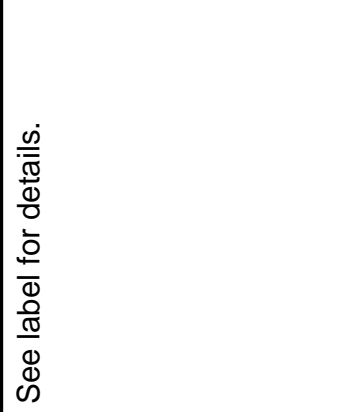 } \\
\hline 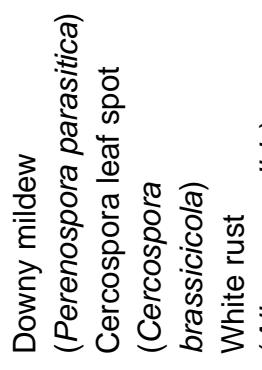 & 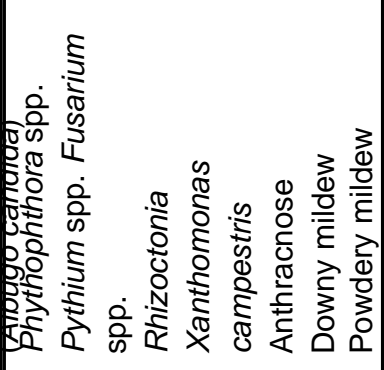 & 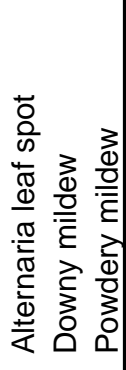 & $\begin{array}{l}3 \\
0 \\
0 \\
\bar{\varepsilon} \\
0 \\
0 \\
0 \\
0 \\
0\end{array}$ & 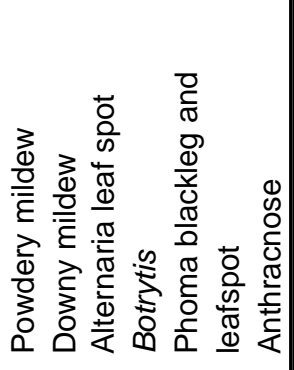 & 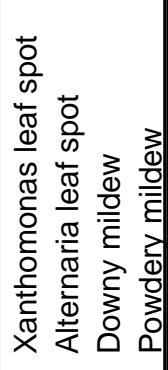 & 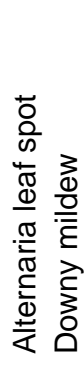 & & \\
\hline$\sim$ & 0 & & & & 0 & $\wedge$ & $\wedge$ & $\wedge$ \\
\hline $\begin{array}{l}0 \\
\stackrel{\dot{N}}{N}\end{array}$ & & & & & 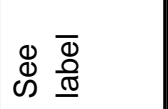 & $\begin{array}{l}\stackrel{\vec{\alpha}}{o} \\
\underline{0}\end{array}$ & $\begin{array}{l}\underline{0} \\
\underline{0}\end{array}$ & $\begin{array}{l}\stackrel{0}{L} \\
\stackrel{0}{+} \\
\leftarrow\end{array}$ \\
\hline $\begin{array}{l}N \\
\stackrel{N}{ } \\
\stackrel{N}{\infty} \\
\infty\end{array}$ & $\begin{array}{l}\bar{\Phi} \\
\bar{\sigma} \\
\Phi \\
\Phi \\
\infty\end{array}$ & 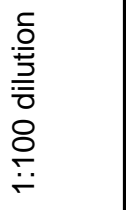 & & $\begin{array}{l}\bar{\Phi} \\
\frac{0}{\sigma} \\
\Phi \\
\Phi\end{array}$ & $\begin{array}{l}\bar{\Phi} \\
\frac{0}{\sigma} \\
\Phi \\
\Phi\end{array}$ & 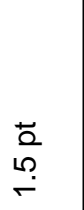 & 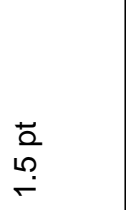 & $\stackrel{\rho}{\stackrel{\rho}{m}}$ \\
\hline$\mp$ & ల్ల & $\stackrel{N}{\Sigma}$ & & ભ్ల & 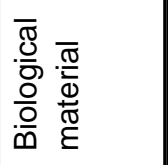 & $\stackrel{L 2}{\Sigma}$ & & \\
\hline 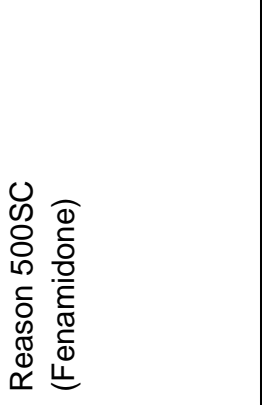 & 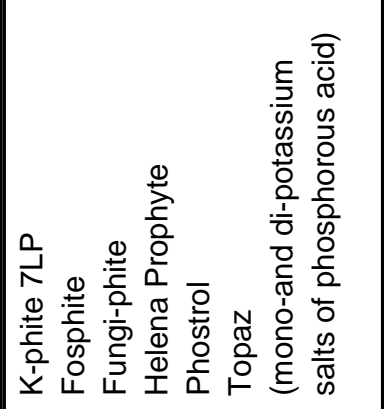 & 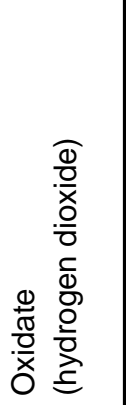 & 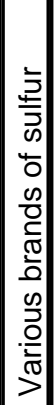 & 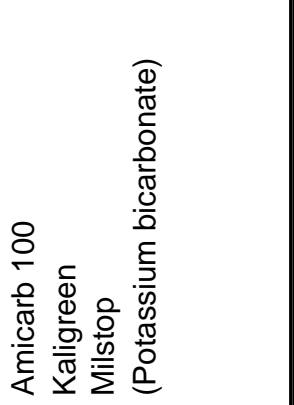 & 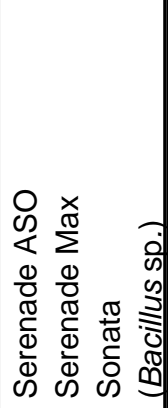 & 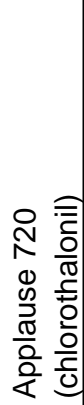 & 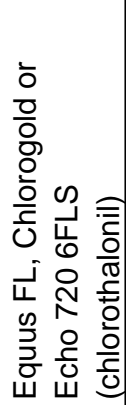 & 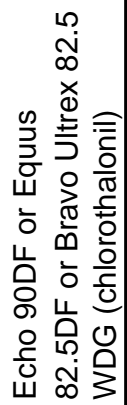 \\
\hline & & & & & & 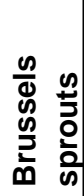 & & \\
\hline
\end{tabular}




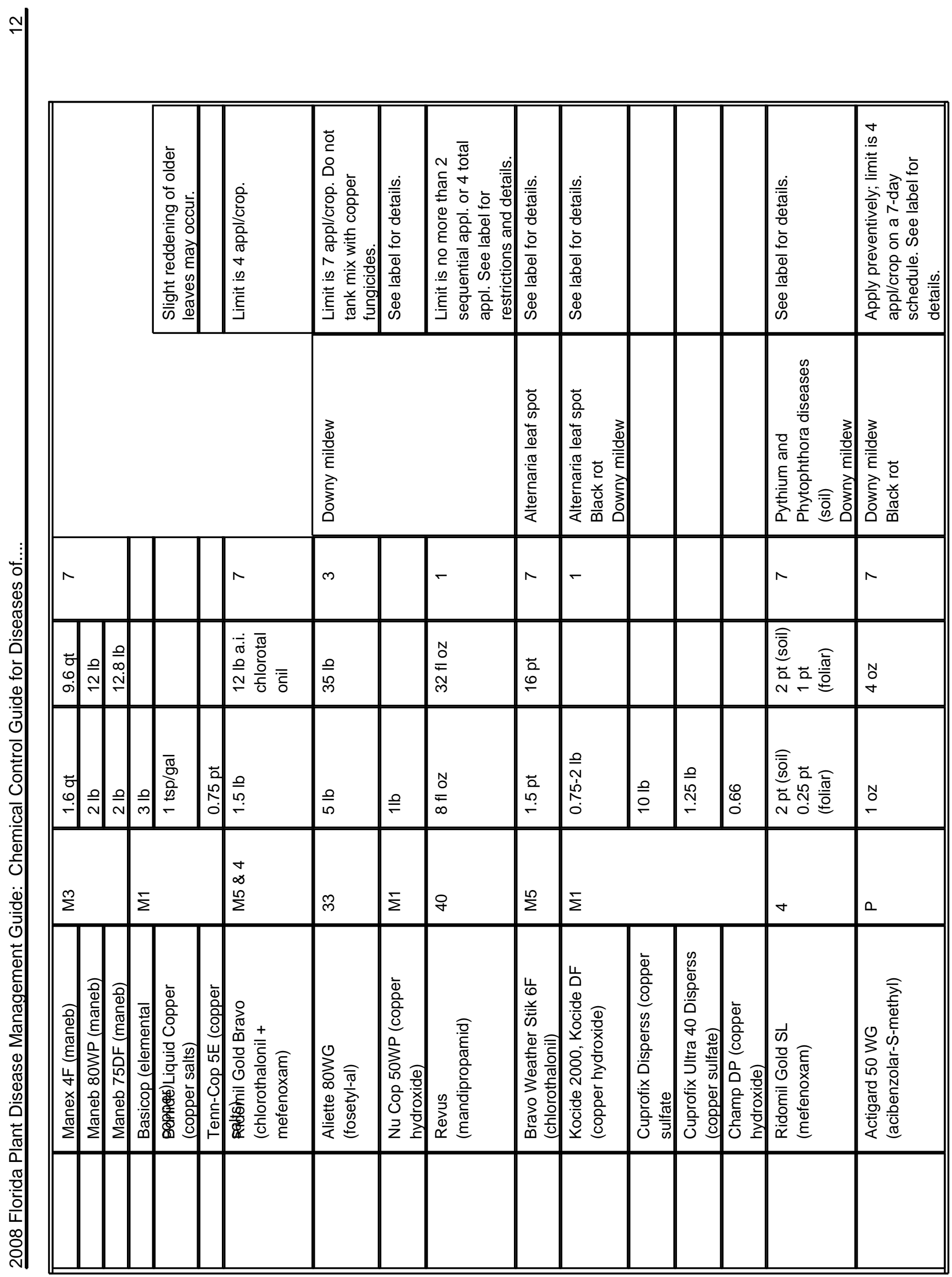




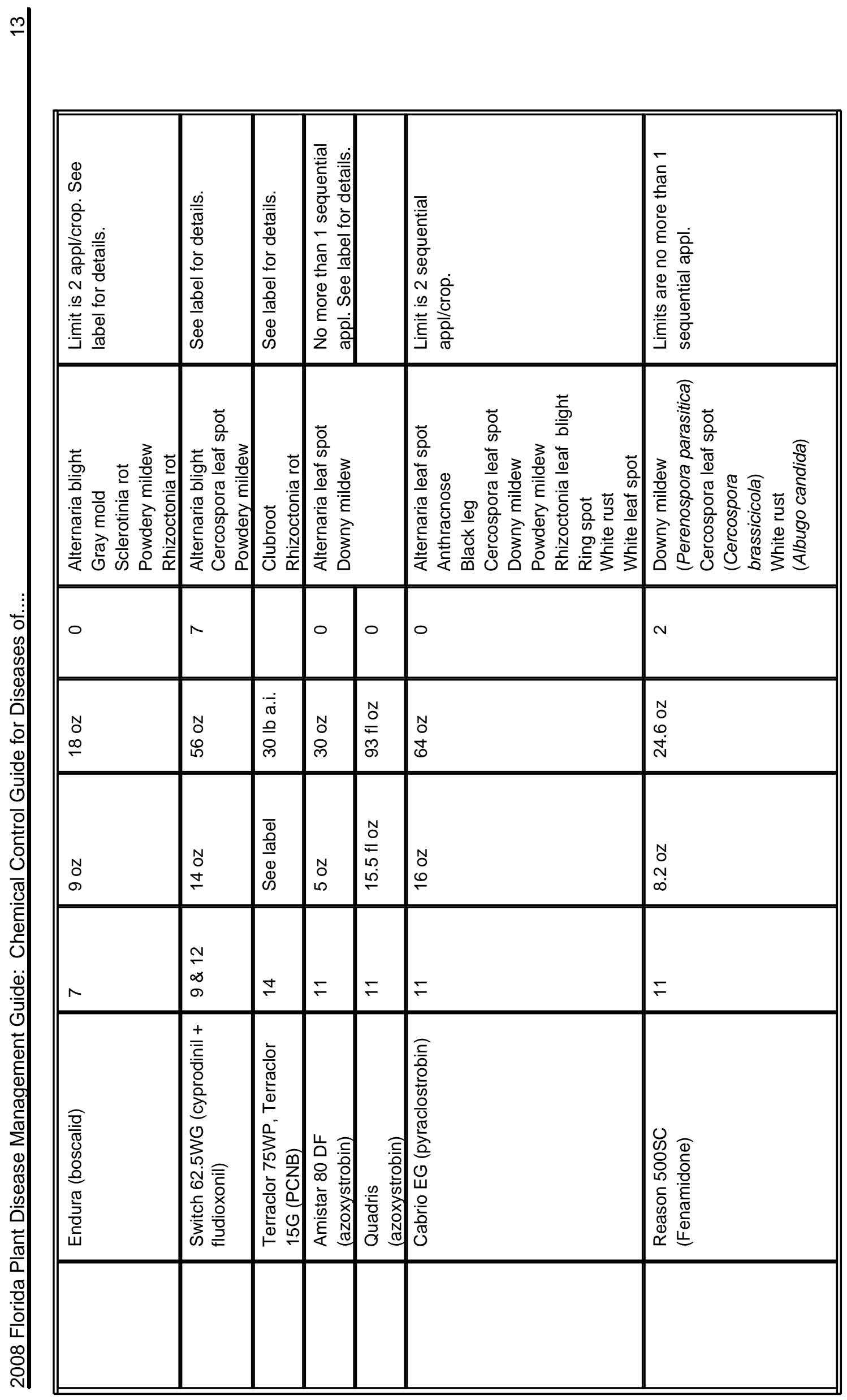




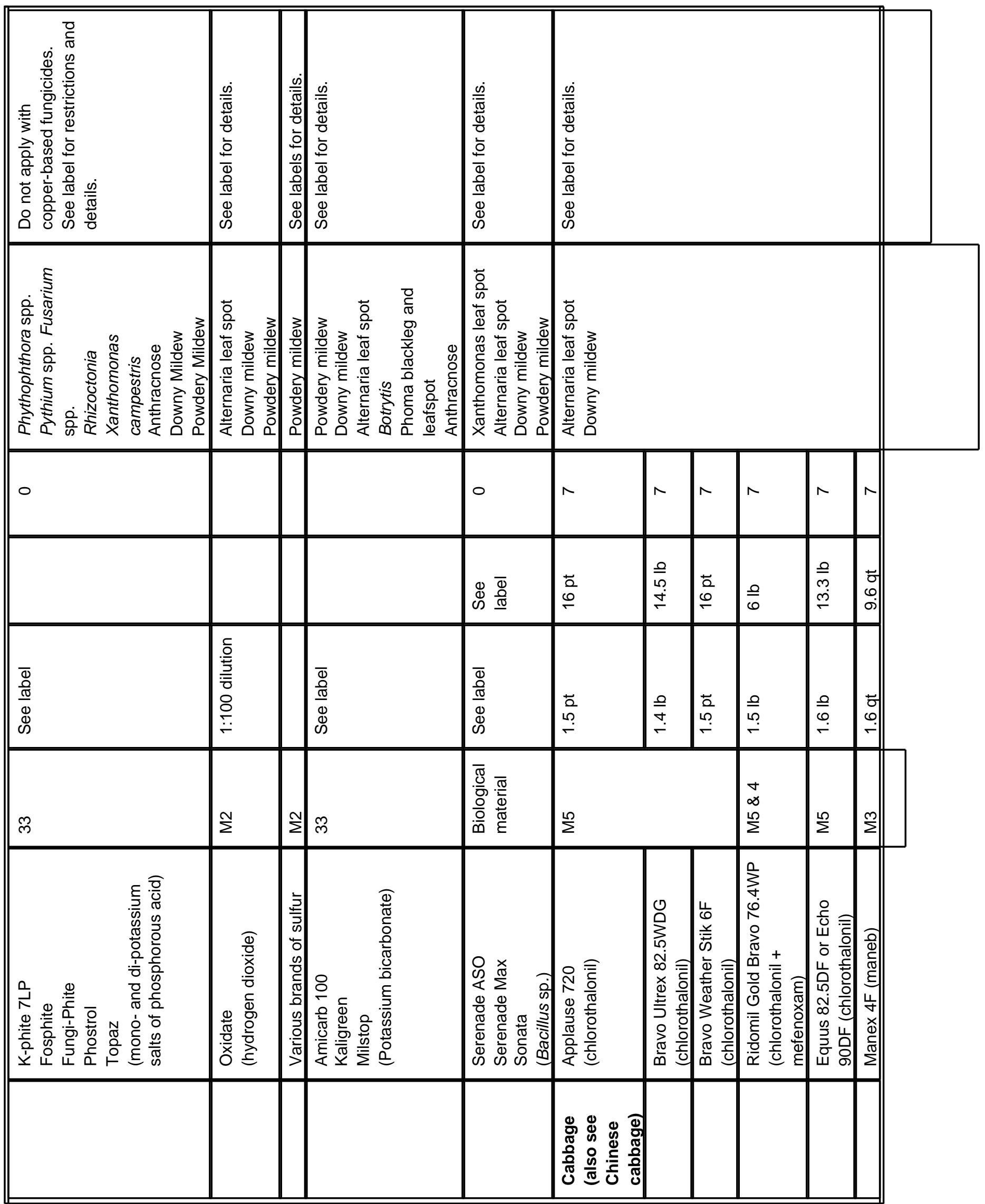




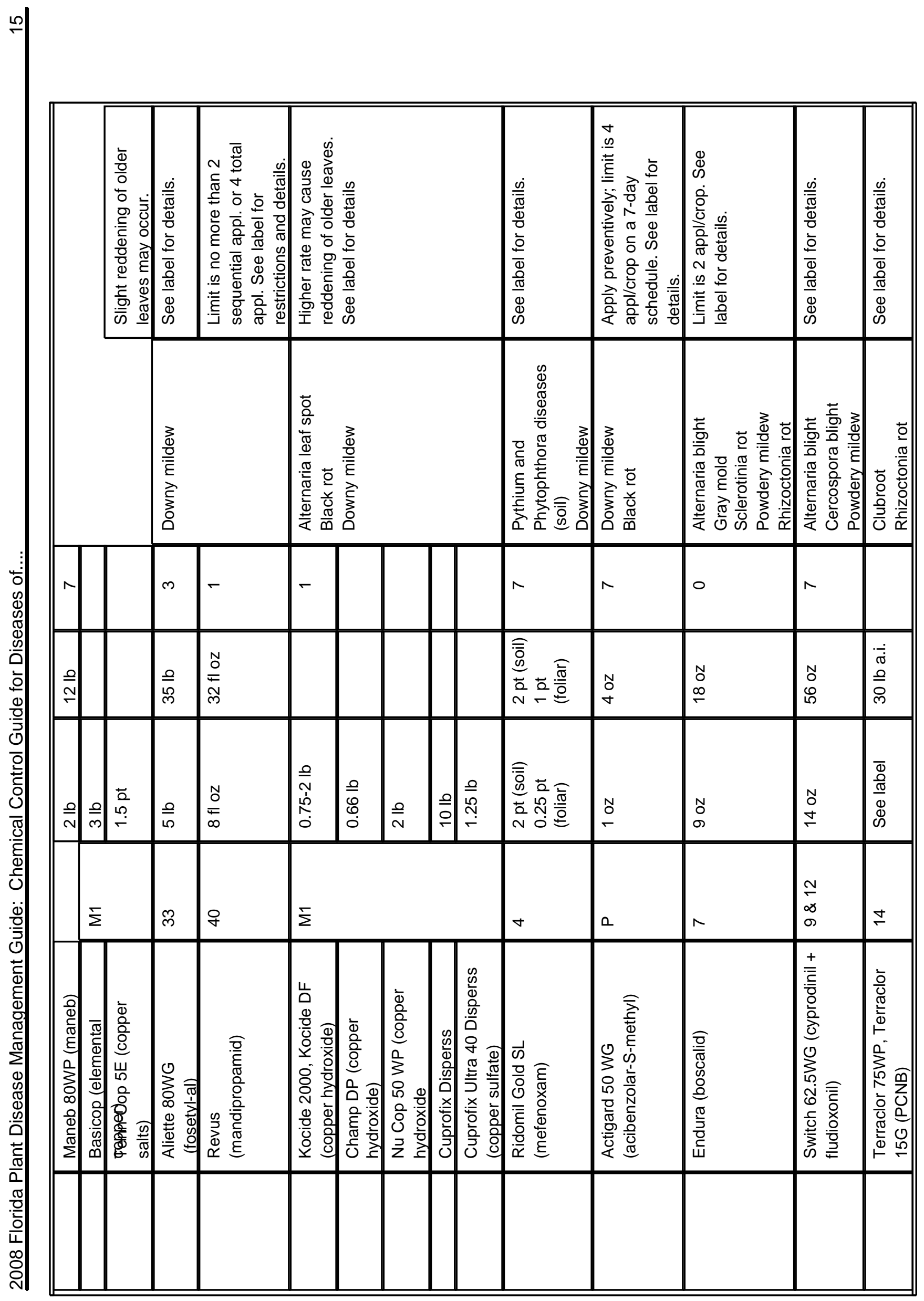




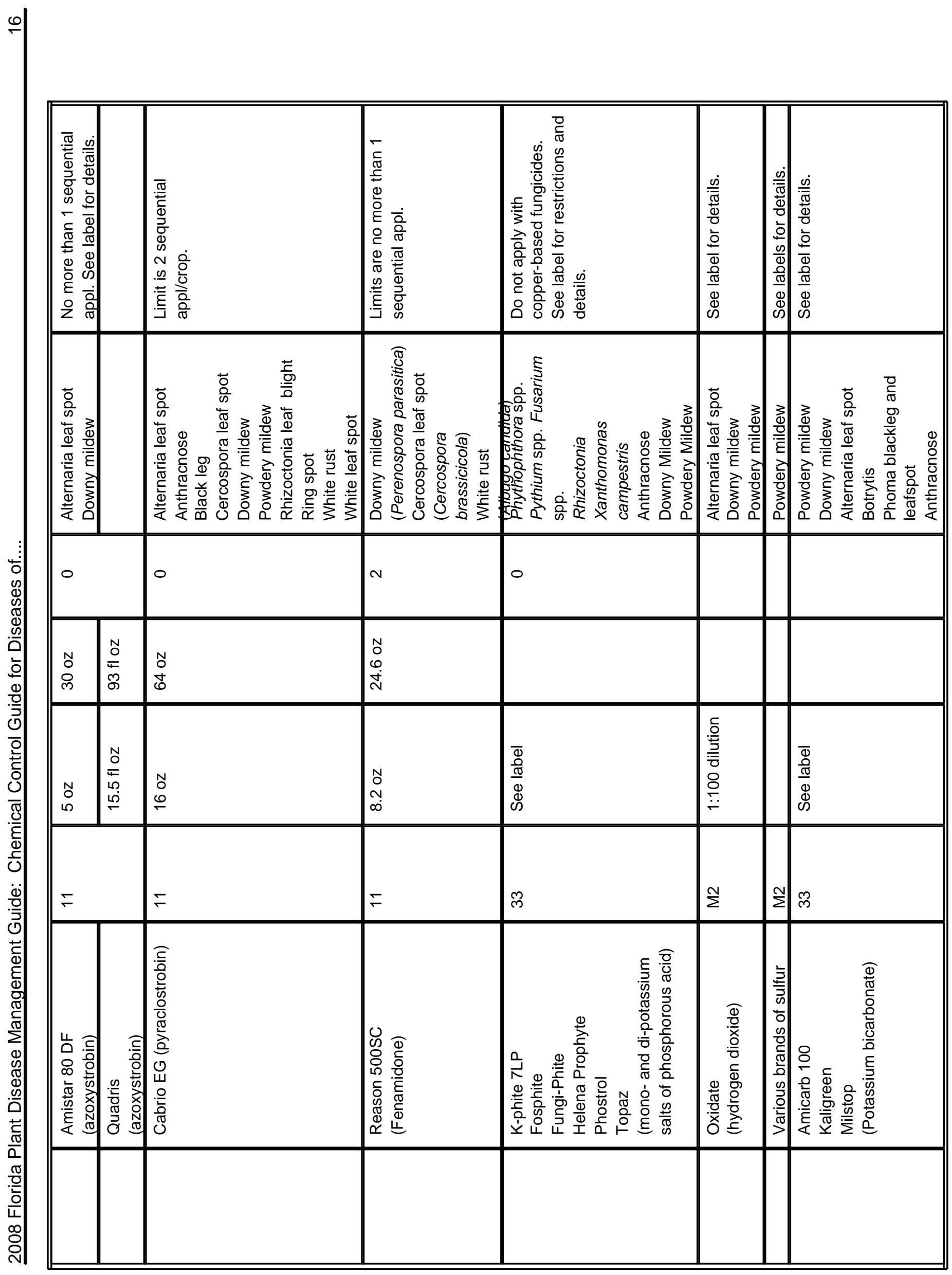




\begin{tabular}{|c|c|c|c|c|c|c|c|}
\hline 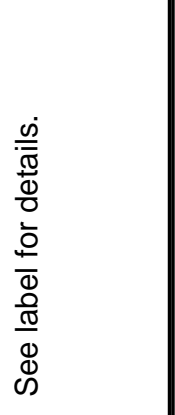 & 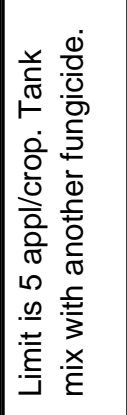 & 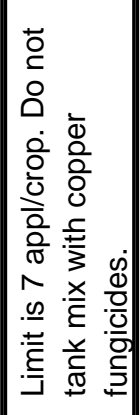 & 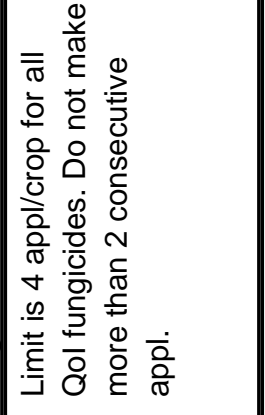 & 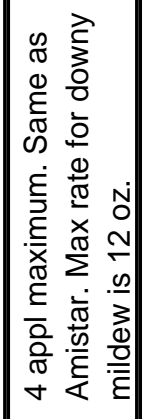 & 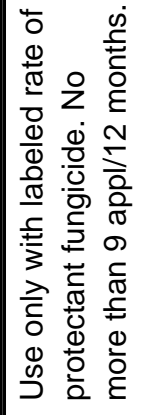 & 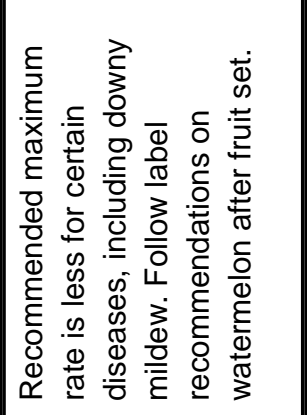 & \\
\hline 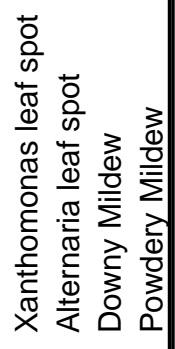 & 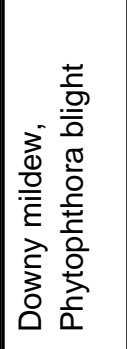 & 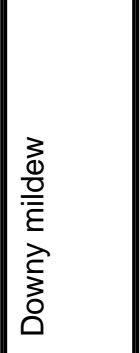 & 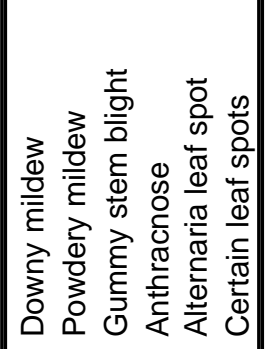 & 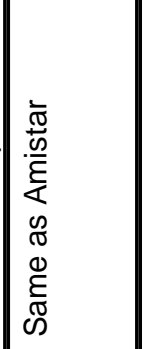 & $\begin{array}{l}3 \\
\frac{3}{0} \\
\frac{0}{\bar{\varepsilon}} \\
2 \\
\frac{1}{3} \\
0 \\
0\end{array}$ & 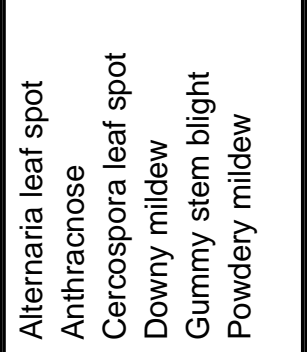 & 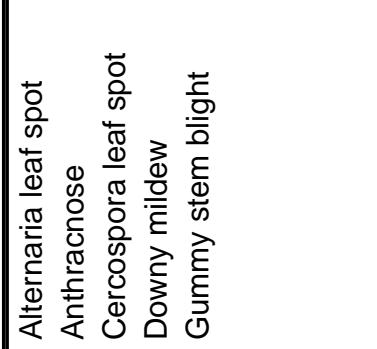 \\
\hline 0 & 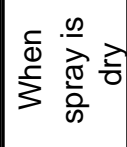 & $\stackrel{i}{\stackrel{\Xi}{N}}$ & - & 0 & $\infty$ & 0 & مـ \\
\hline 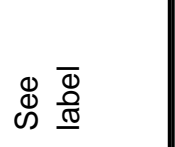 & $\begin{array}{l}\text { No } \\
\text { N } \\
\text { N }\end{array}$ & $\stackrel{\circ}{\circ}$ & $\begin{array}{l}\stackrel{0}{0} \\
\infty \\
\infty \\
-\end{array}$ & $\begin{array}{l}N \\
\stackrel{N}{E} \\
\dot{J}\end{array}$ & 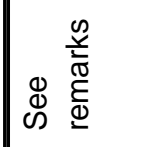 & 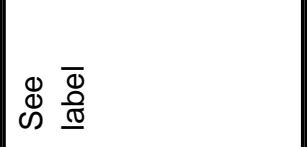 & 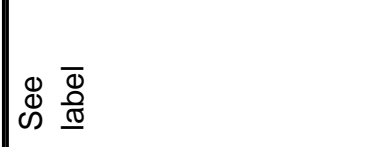 \\
\hline $\begin{array}{l}\bar{\Phi} \\
\frac{0}{\sigma} \\
\Phi \\
\Phi \\
\text { Ф) }\end{array}$ & $\begin{array}{l}N \\
0 \\
+ \\
0\end{array}$ & $\frac{0}{10}$ & 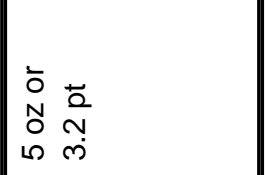 & $\begin{array}{l}\stackrel{N}{O} \\
\frac{E}{6} \\
\underline{0}\end{array}$ & $\begin{array}{l}N \\
\stackrel{N}{ } \\
N \\
M\end{array}$ & $\begin{array}{l}\bar{\Phi} \\
\frac{0}{\sigma} \\
\Phi \\
\mathscr{D}\end{array}$ & $\begin{array}{l}\bar{\Phi} \\
\frac{\pi}{\pi} \\
\Phi \\
\Phi\end{array}$ \\
\hline 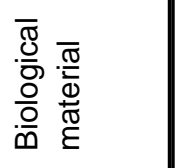 & 우 & ঙ্ল & $\mp$ & & $\hat{N}$ & $\stackrel{10}{\Sigma}$ & $\sum^{M}$ \\
\hline 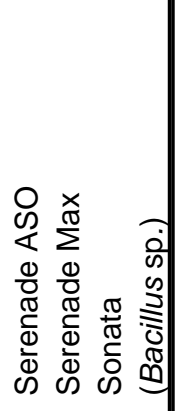 & 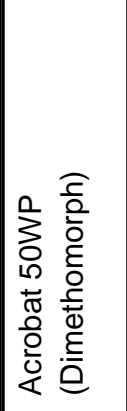 & 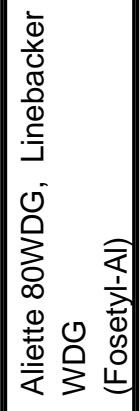 & 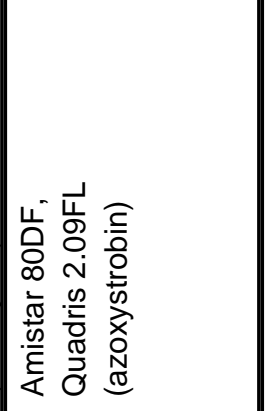 & 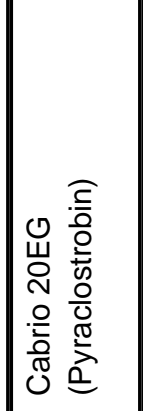 & 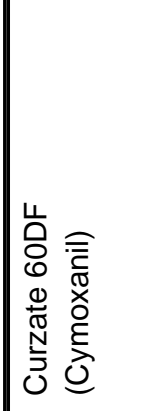 & 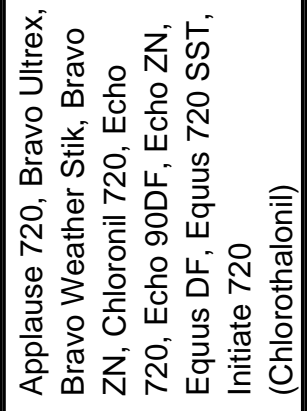 & 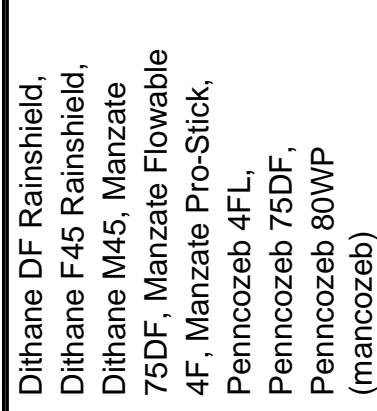 \\
\hline & 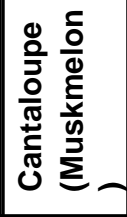 & & & & & & \\
\hline
\end{tabular}




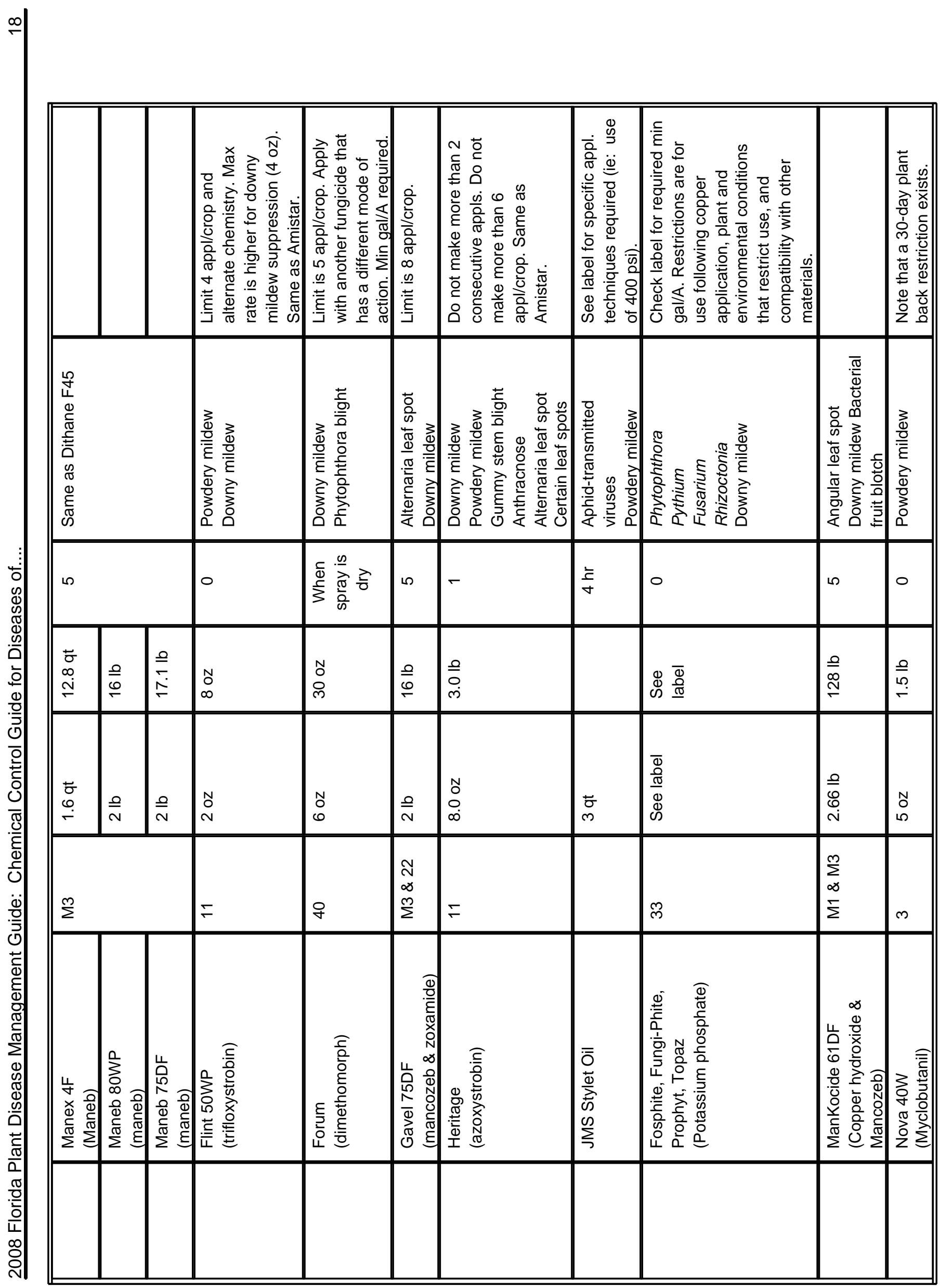




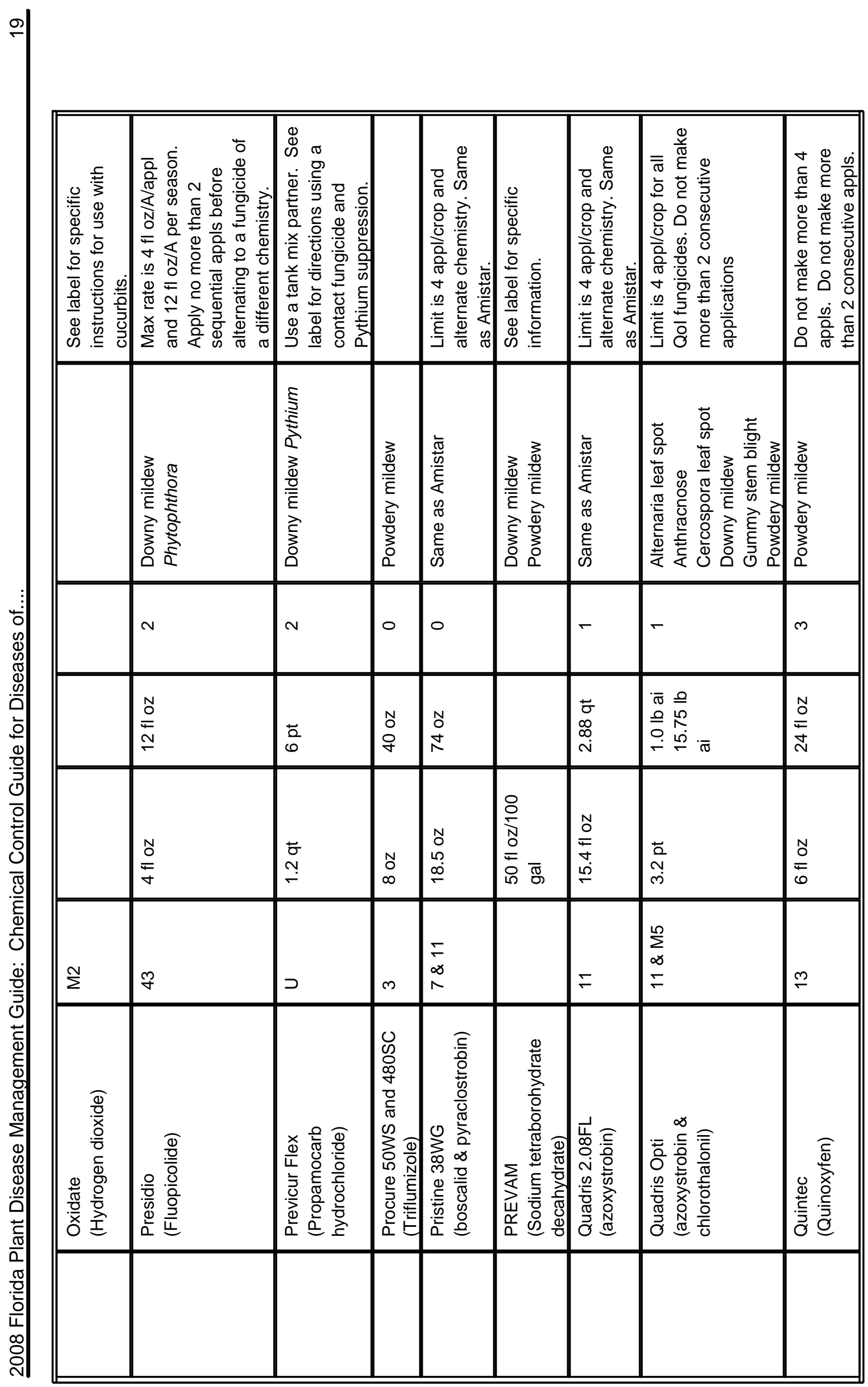




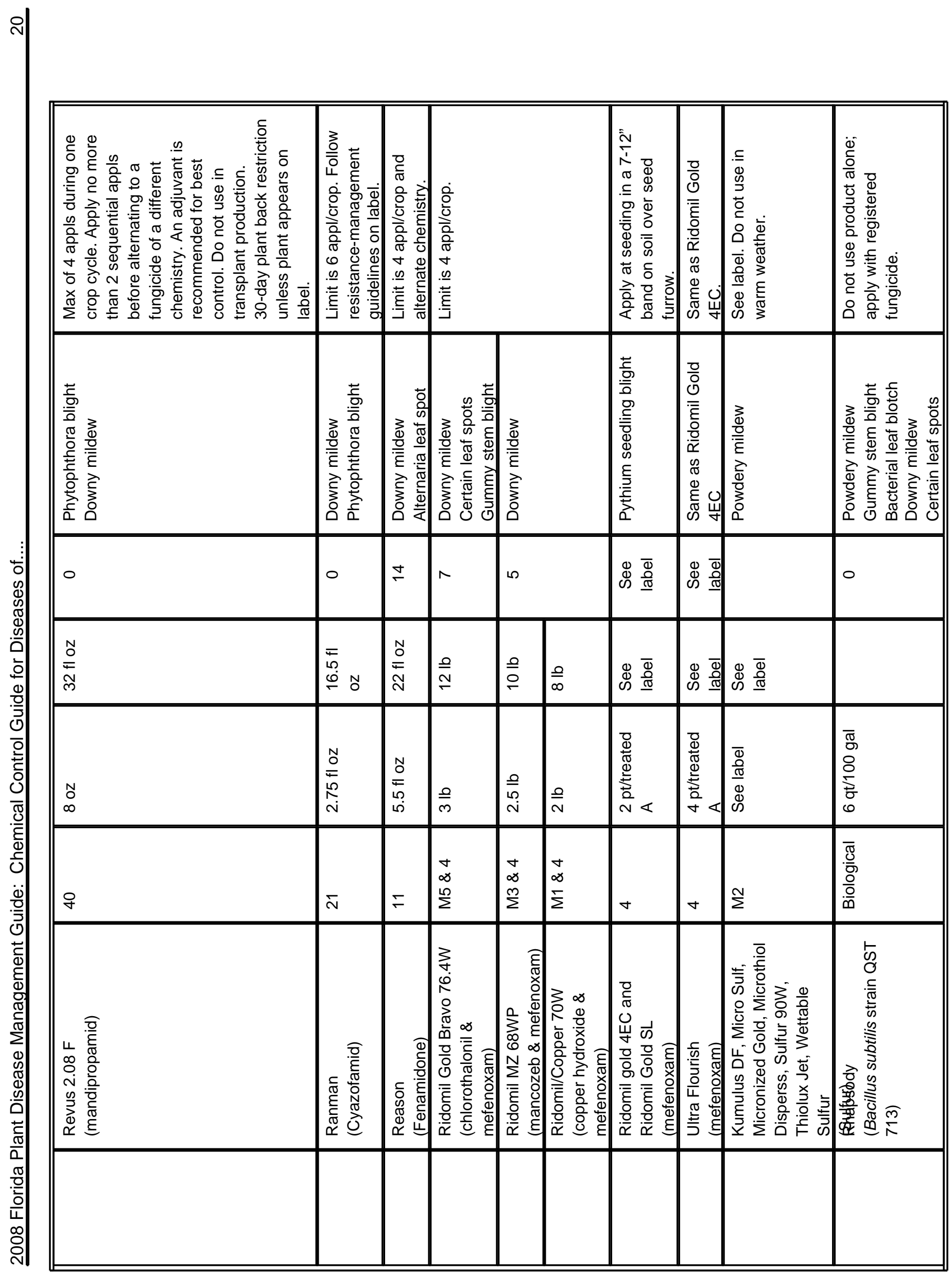




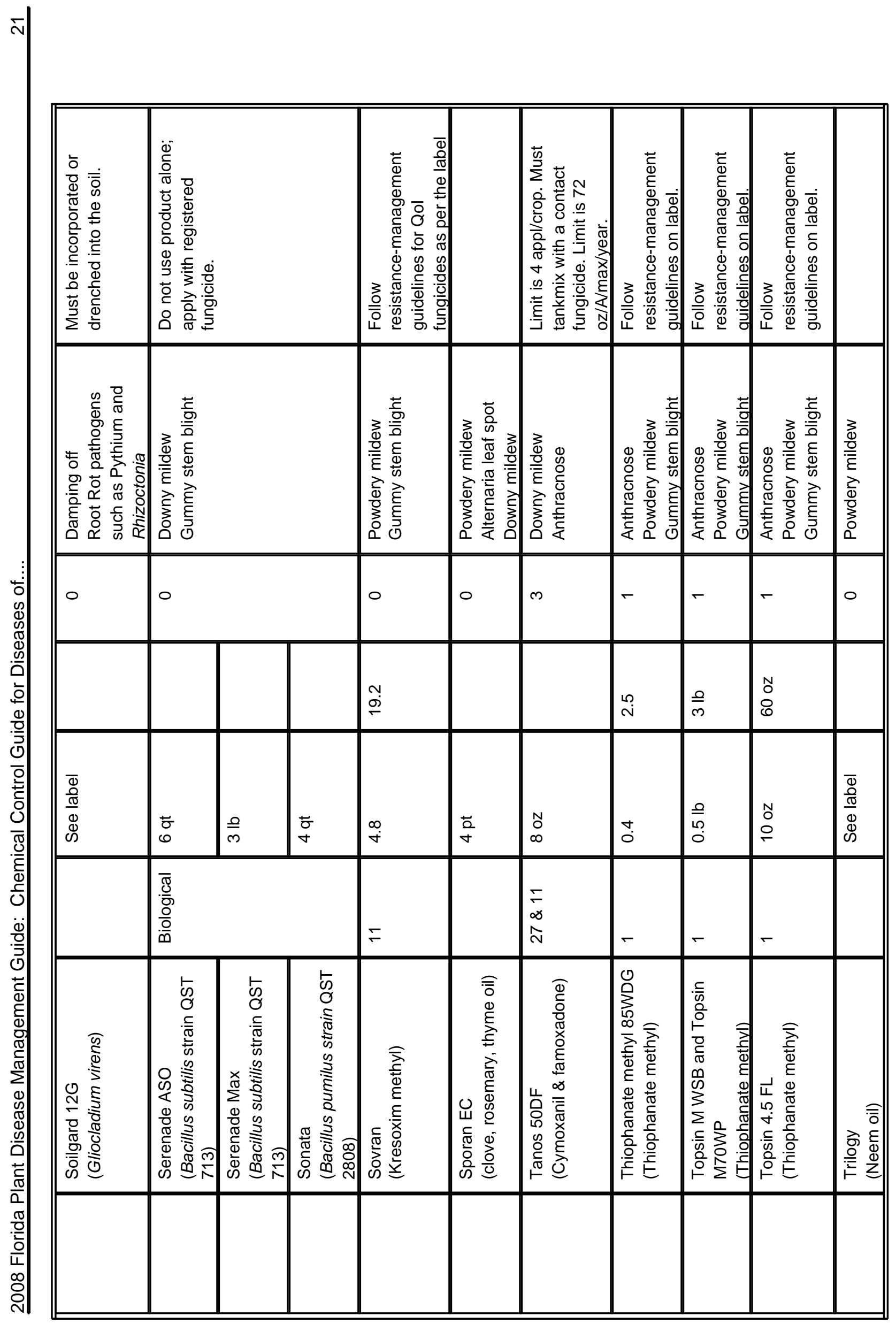




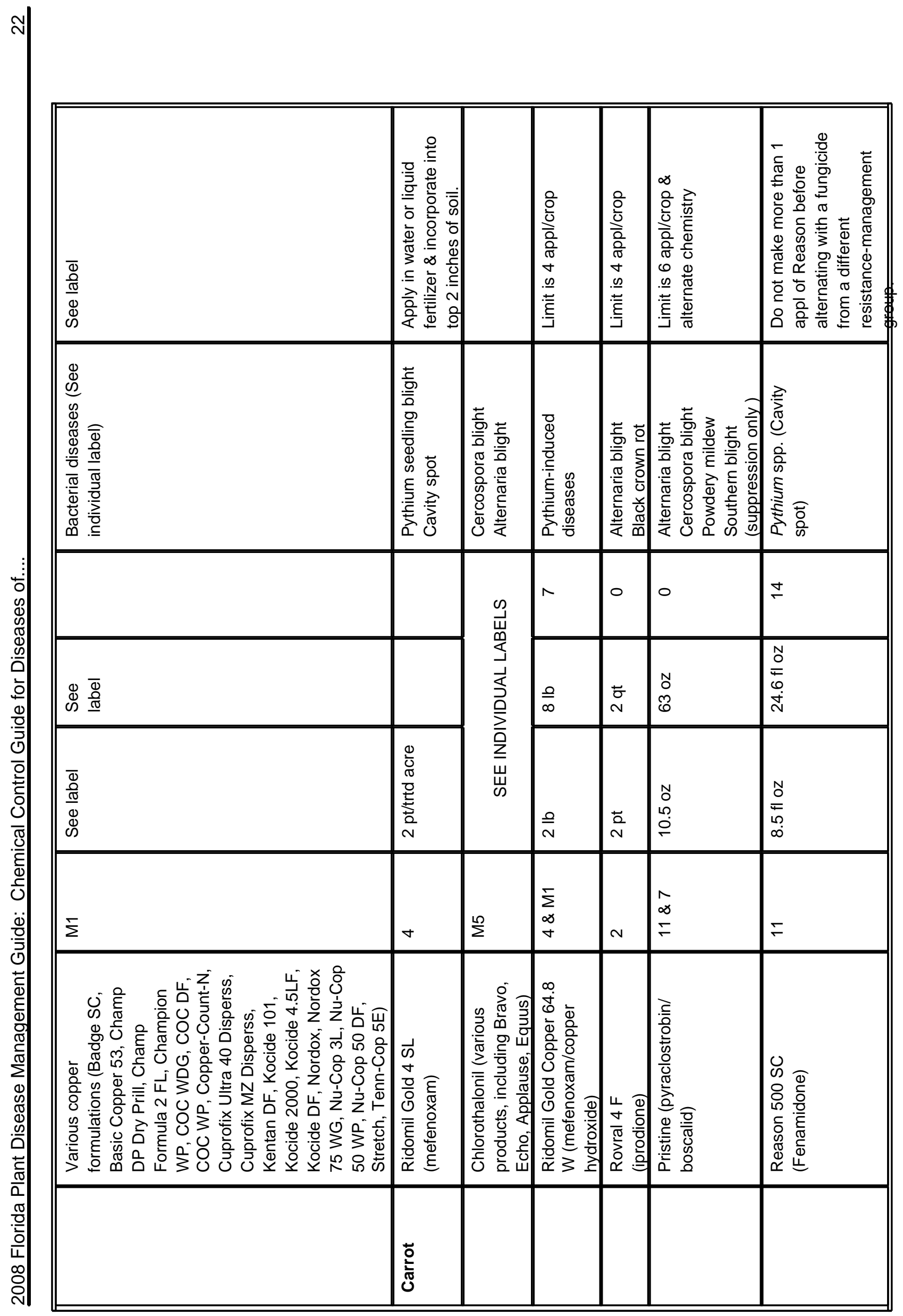




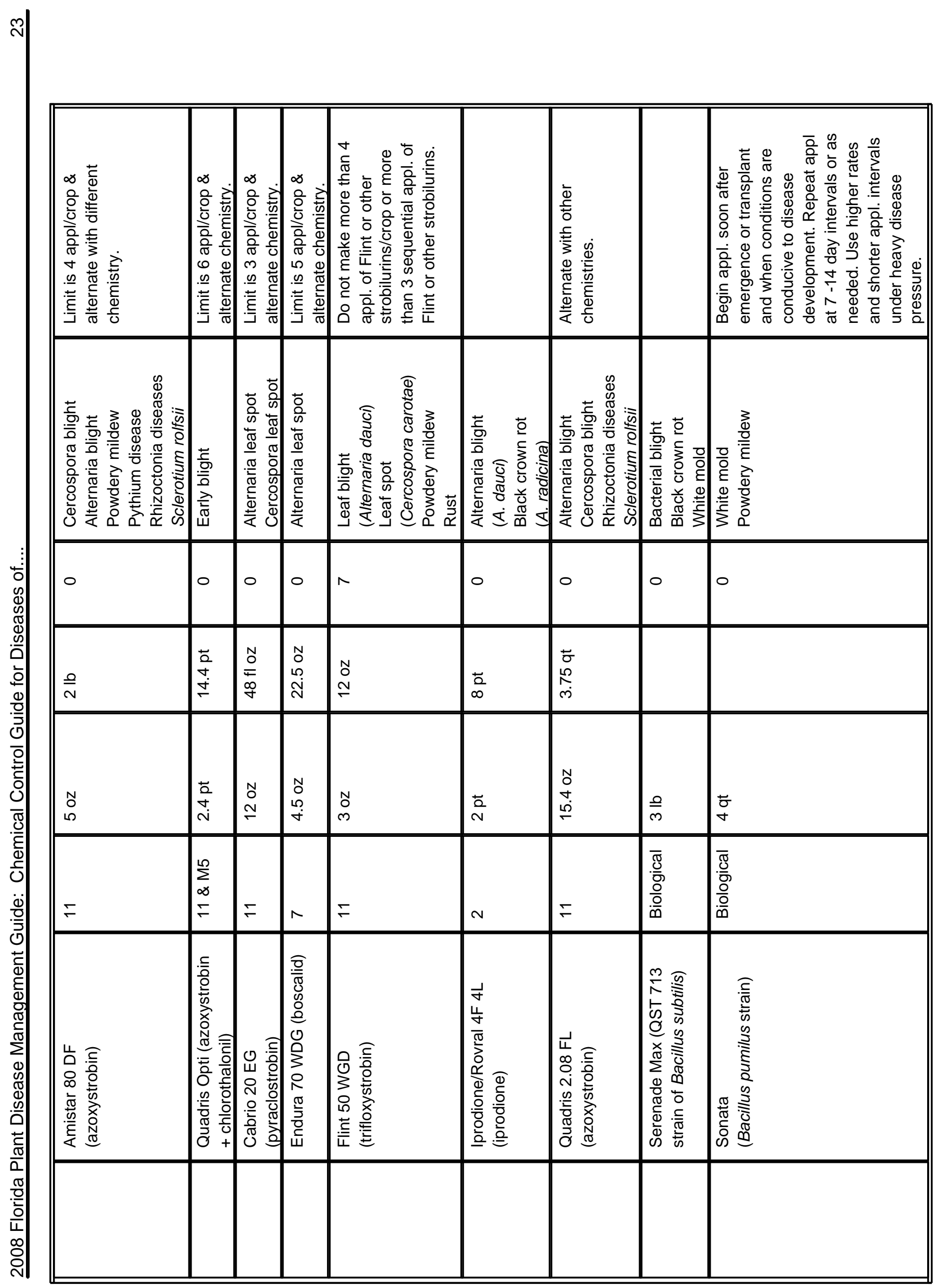




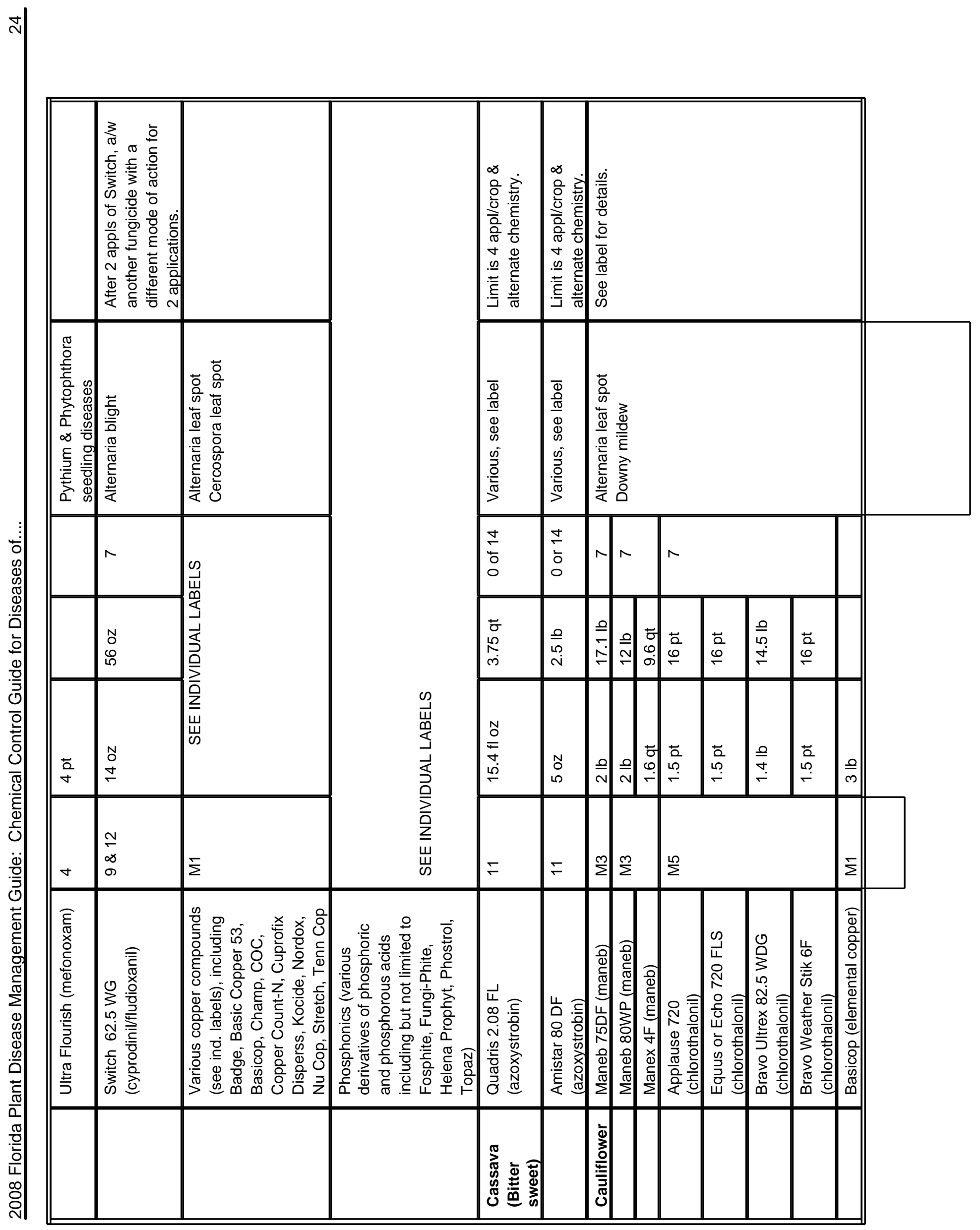




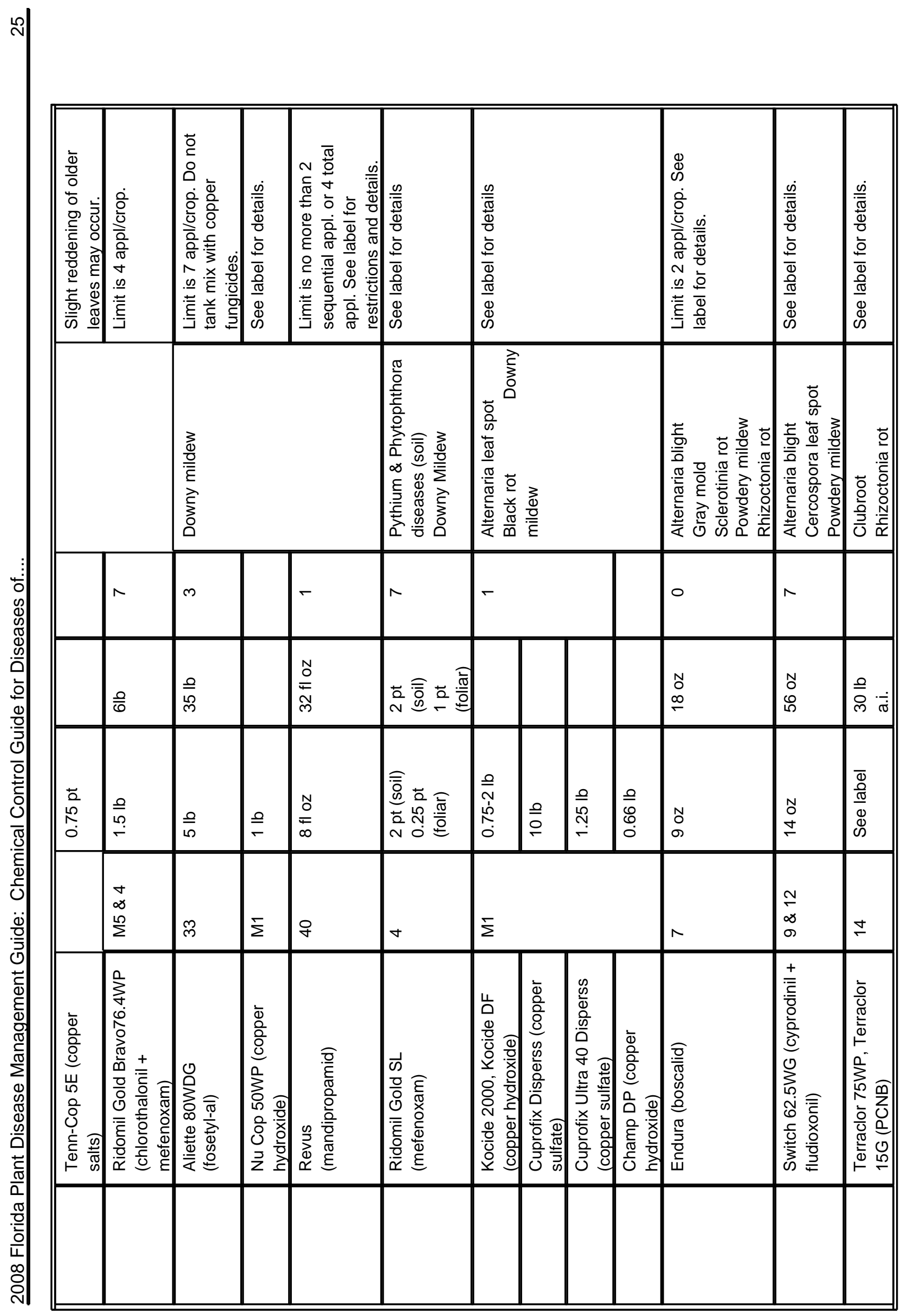




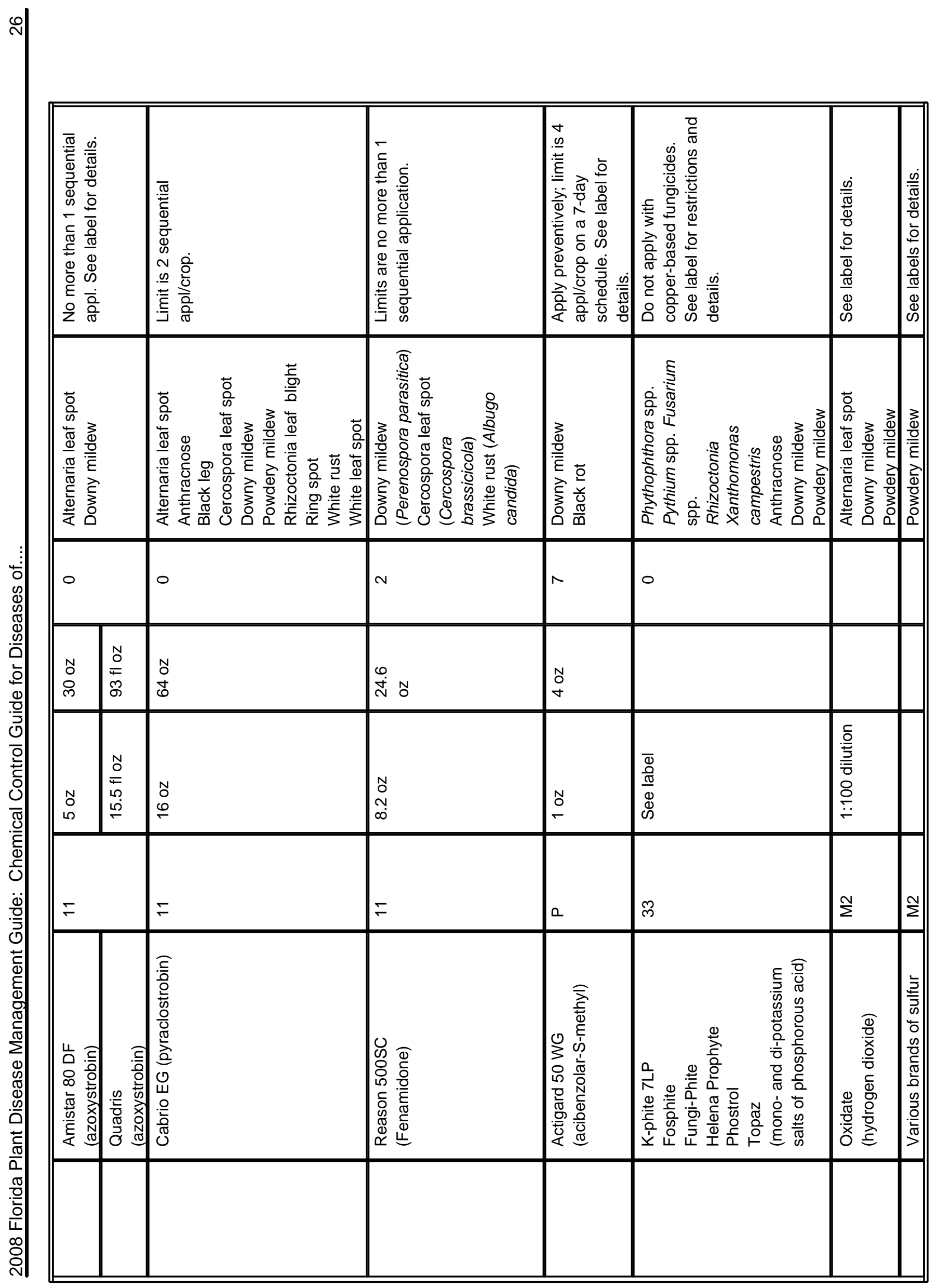




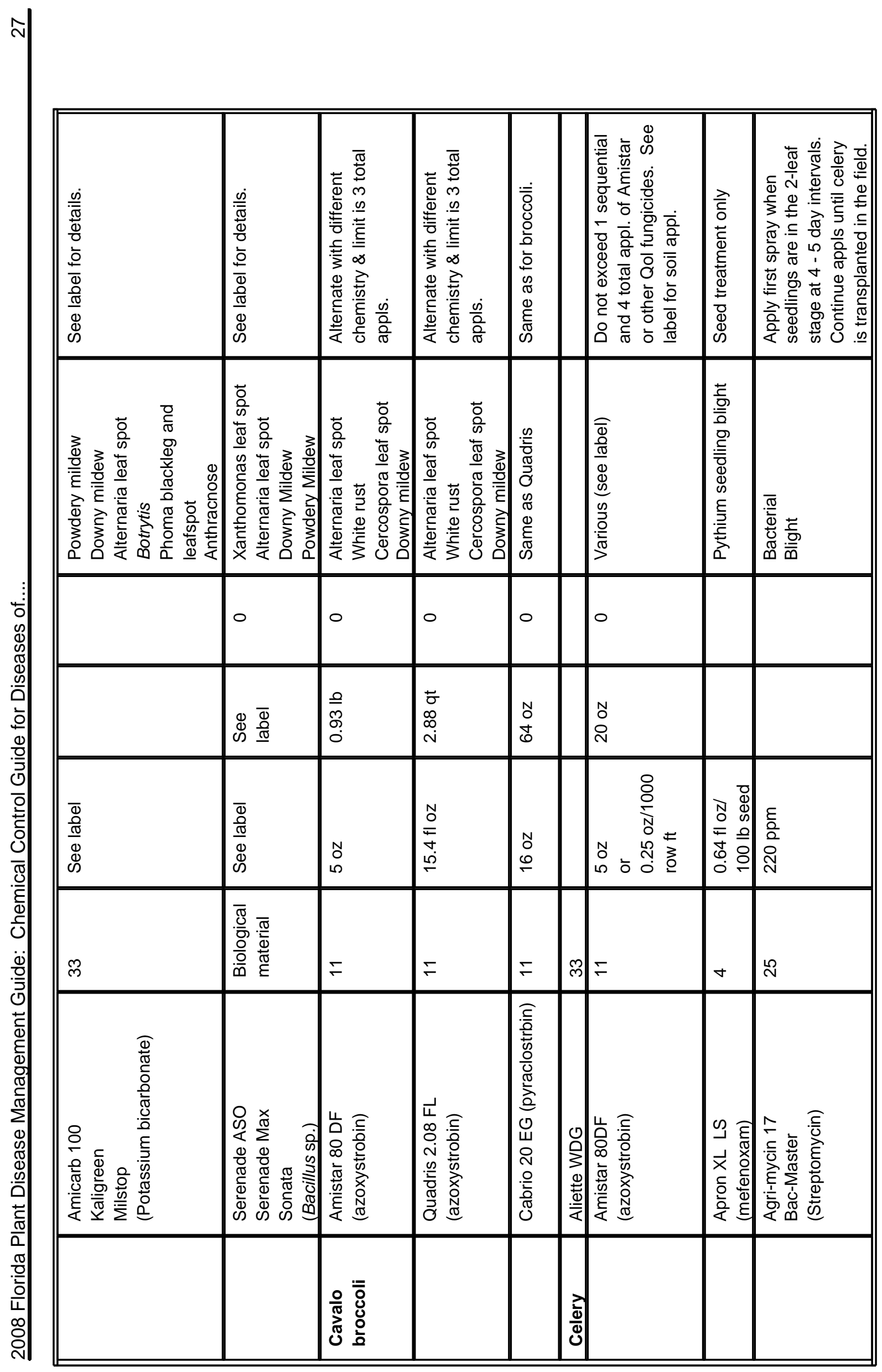




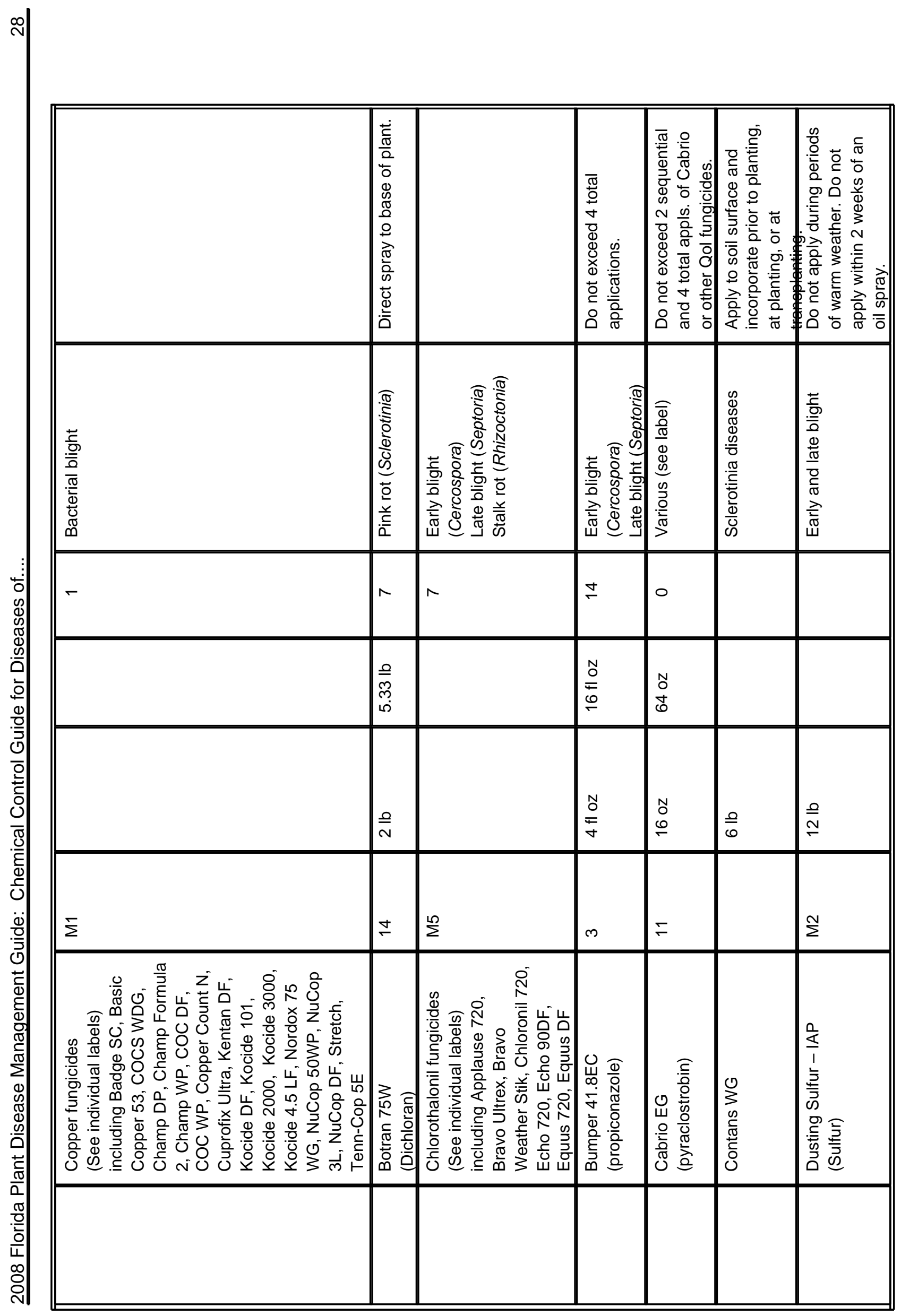




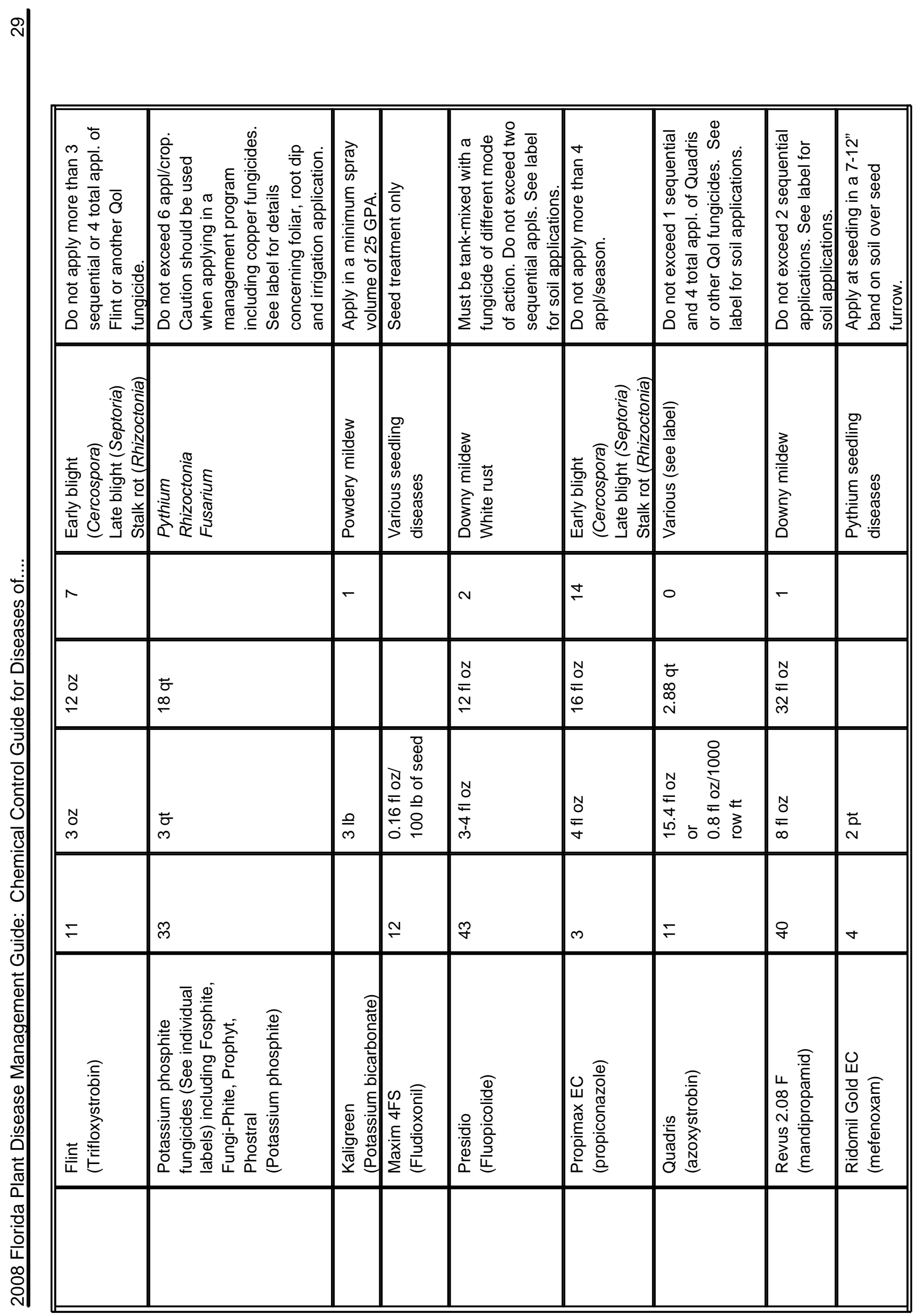




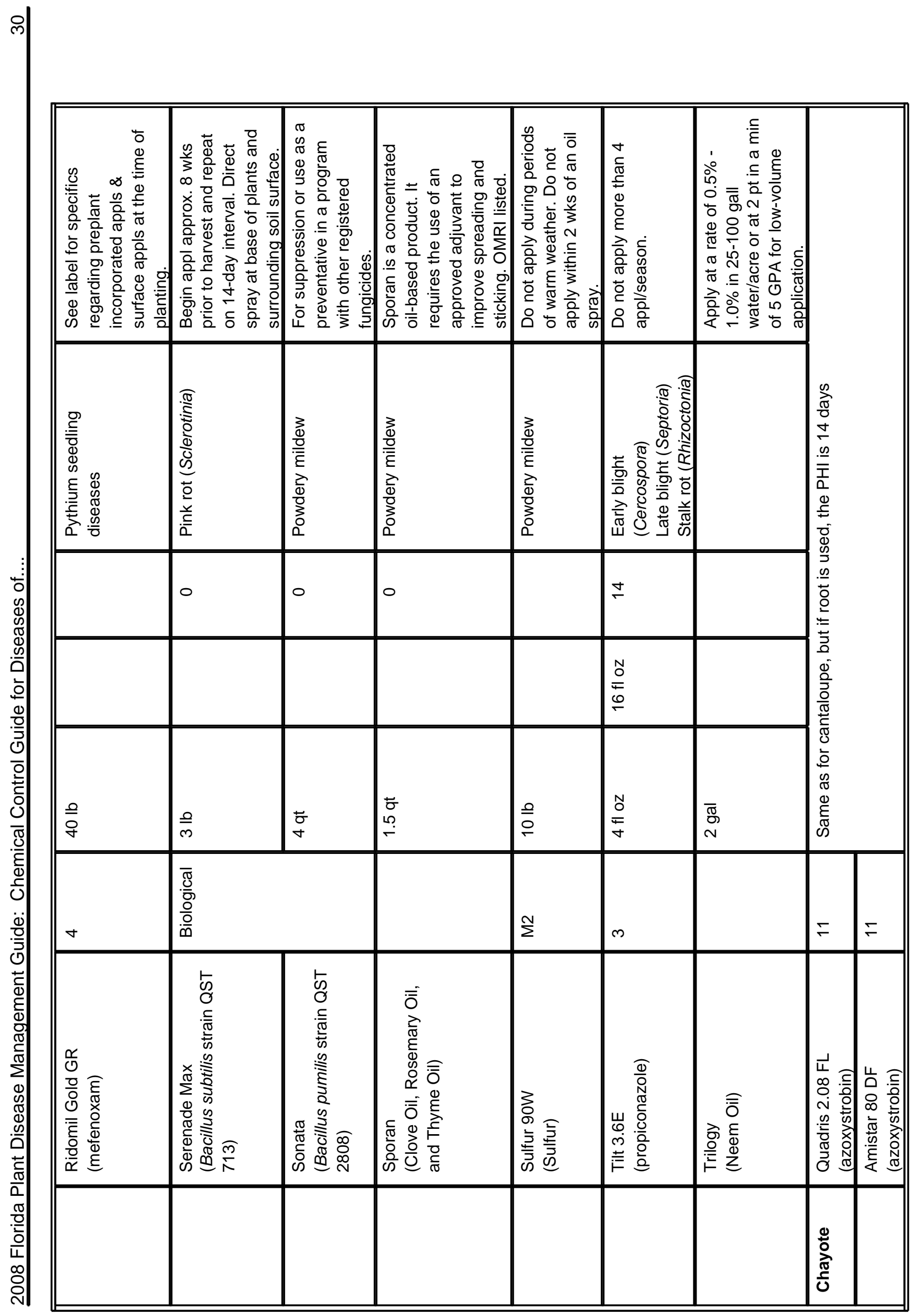




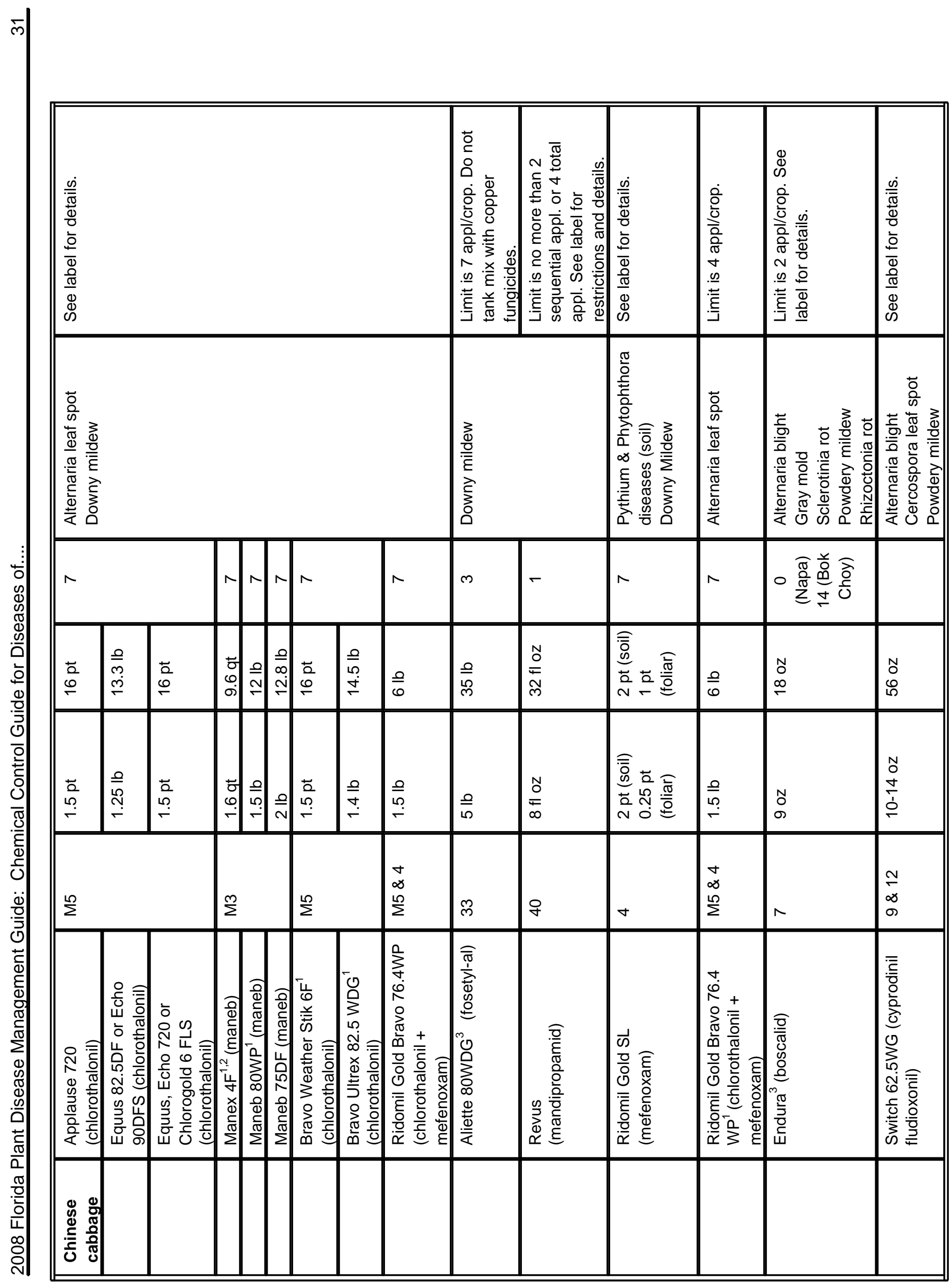




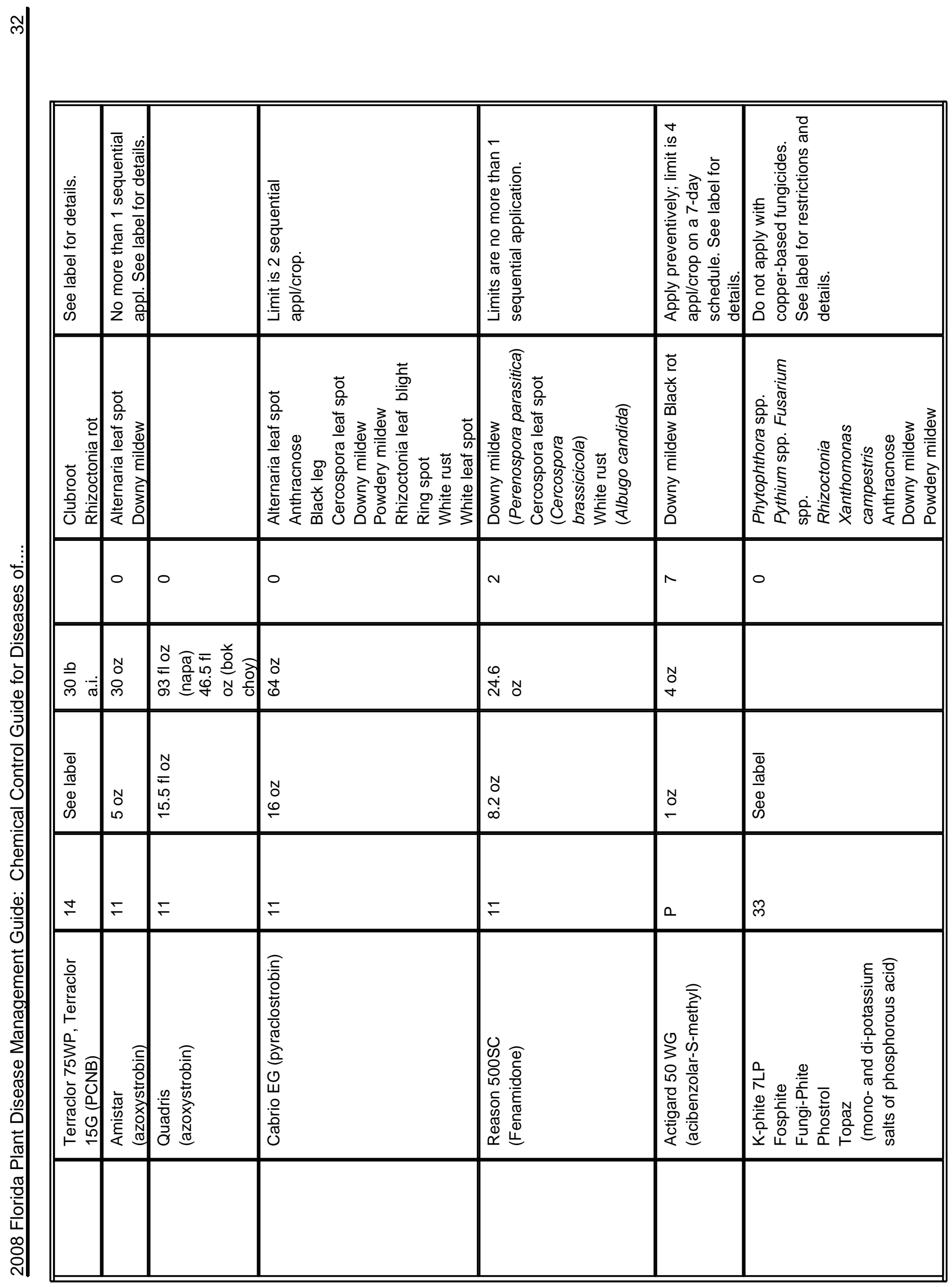




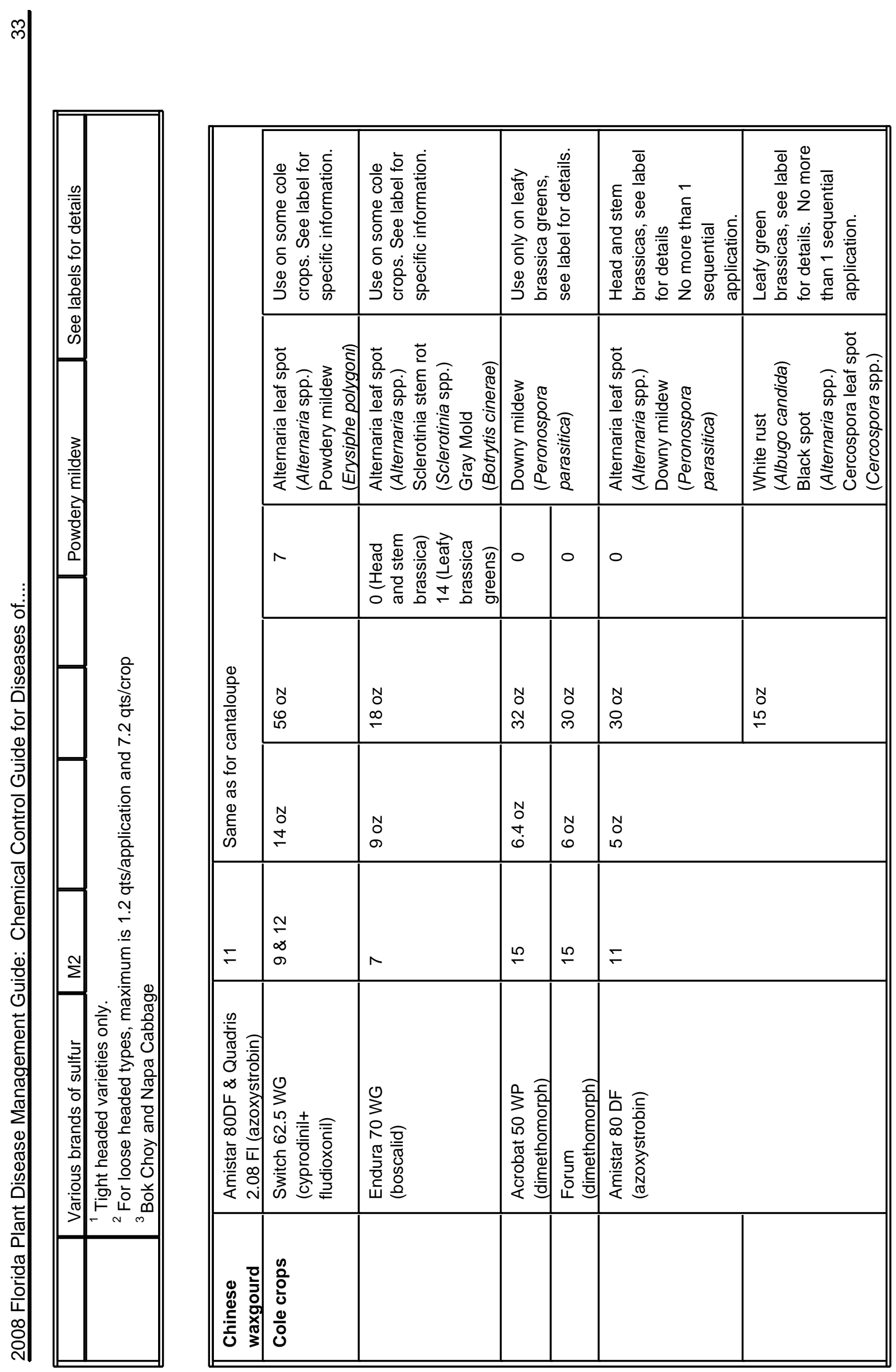




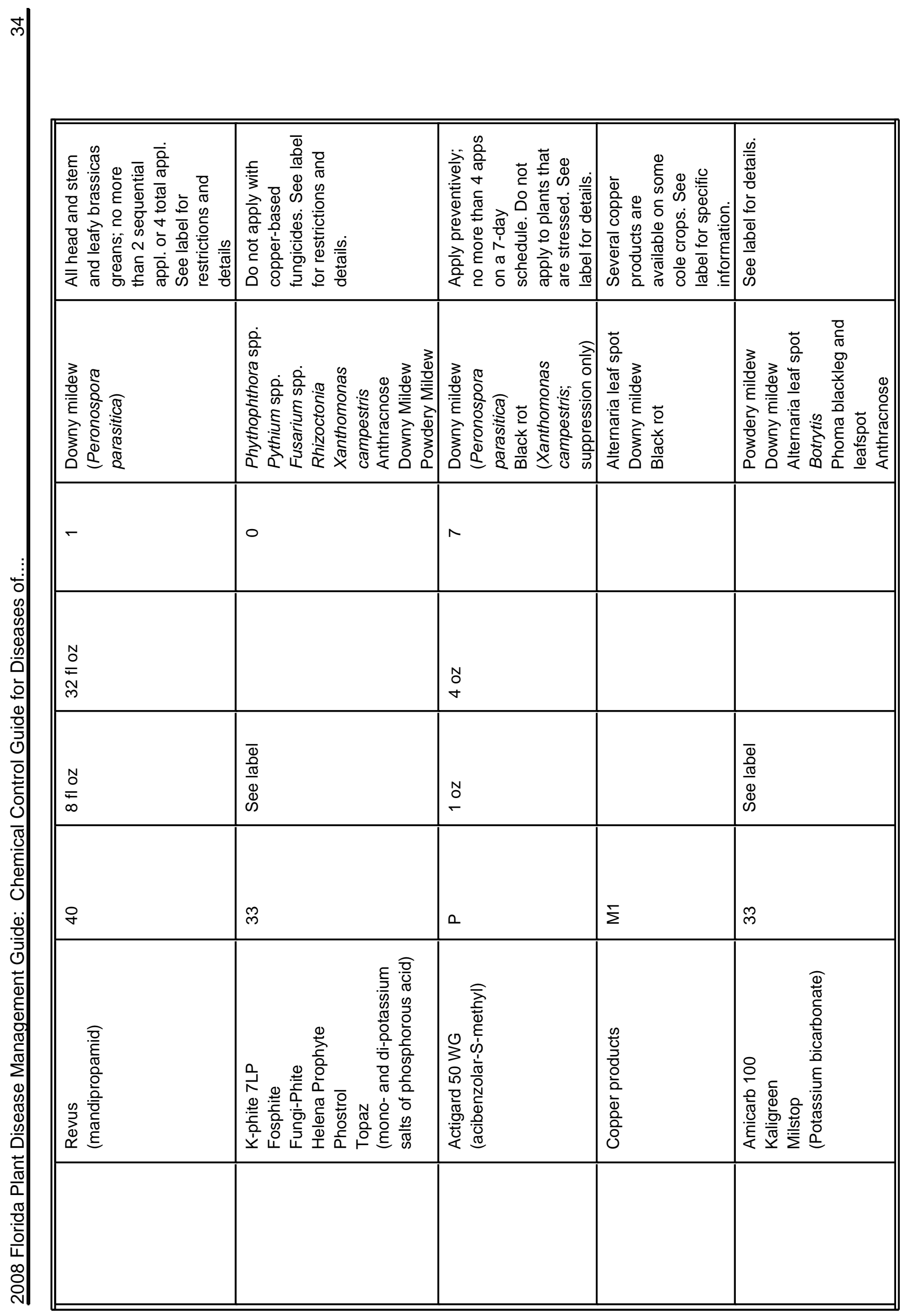




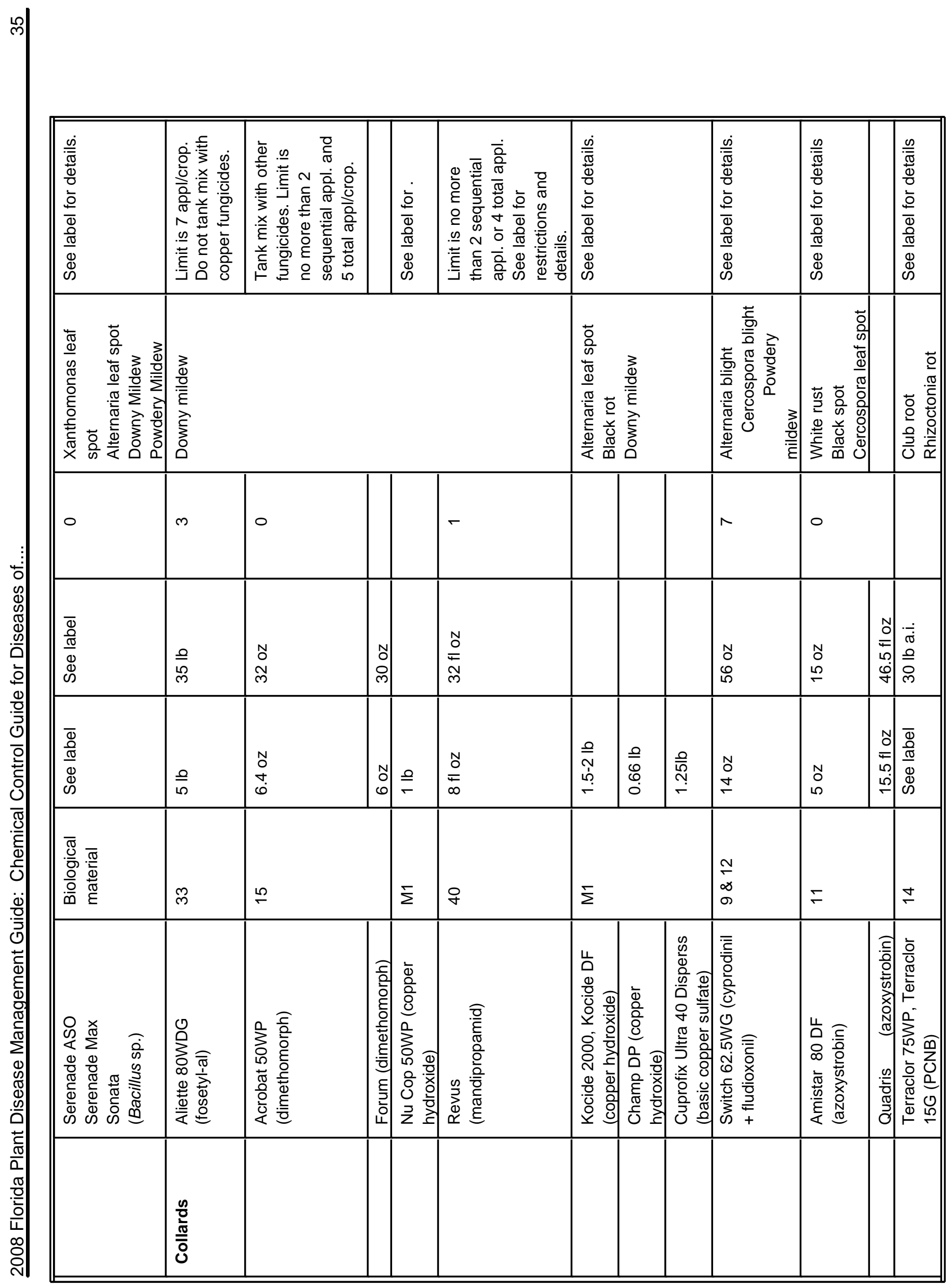




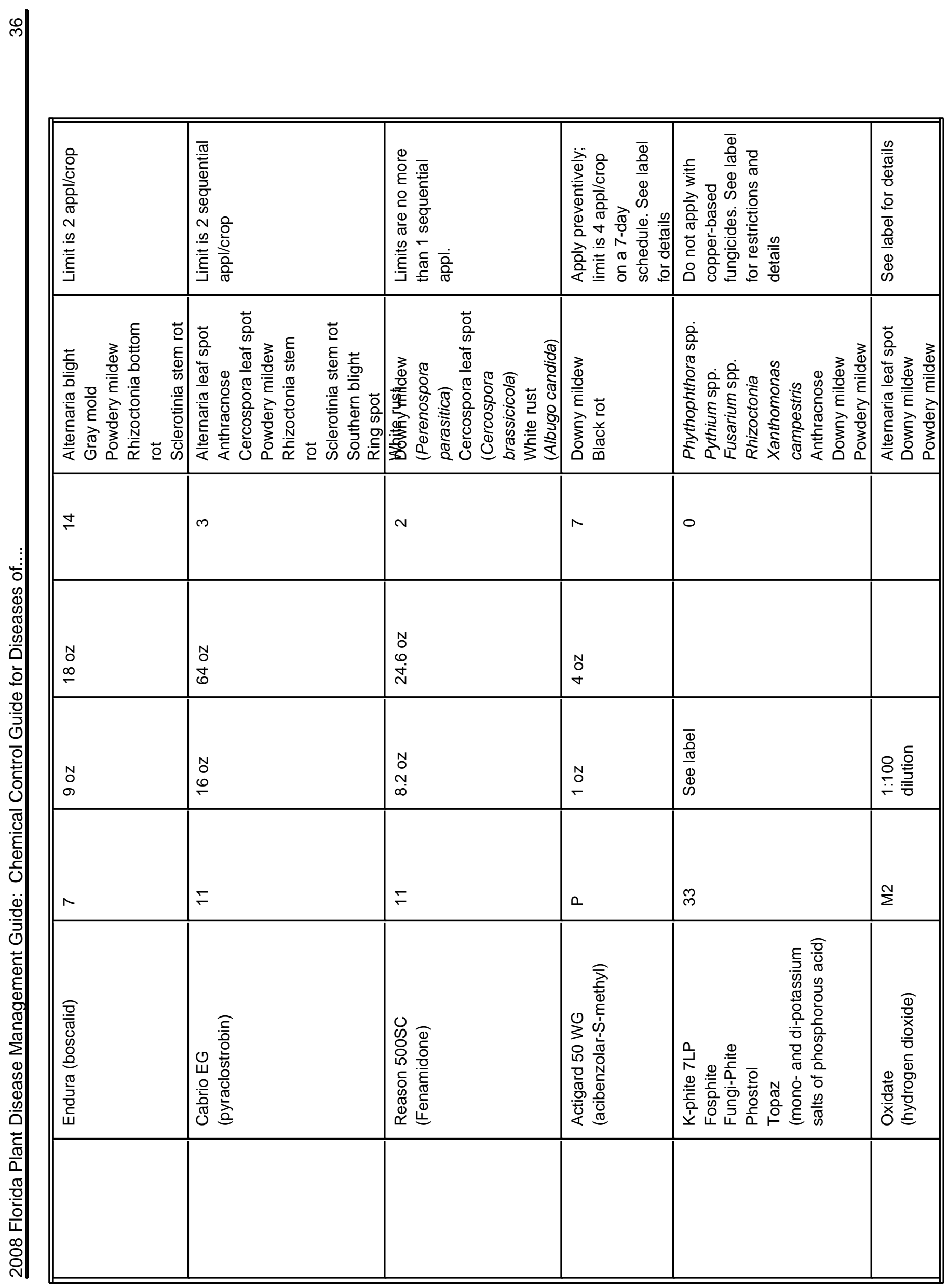




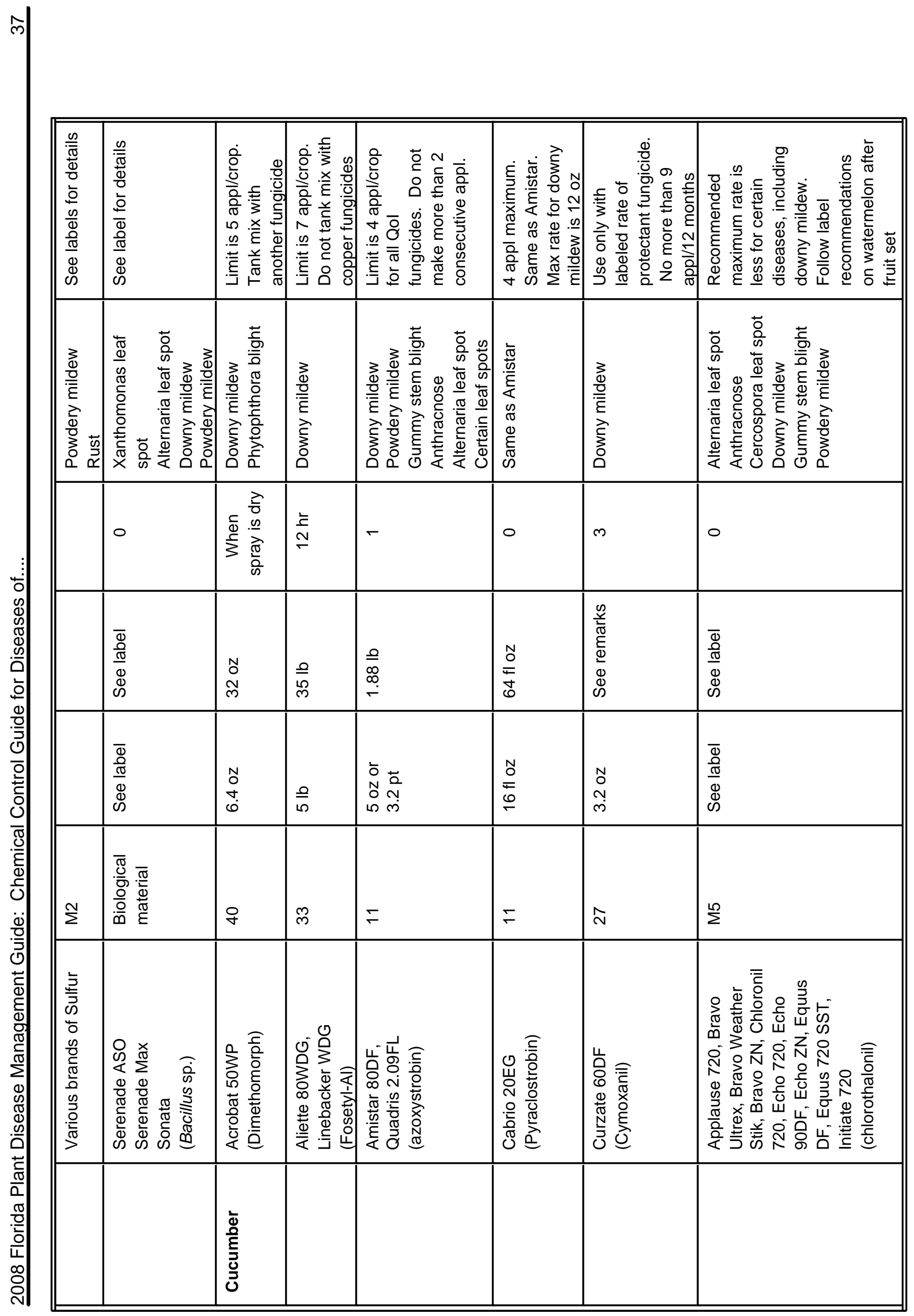




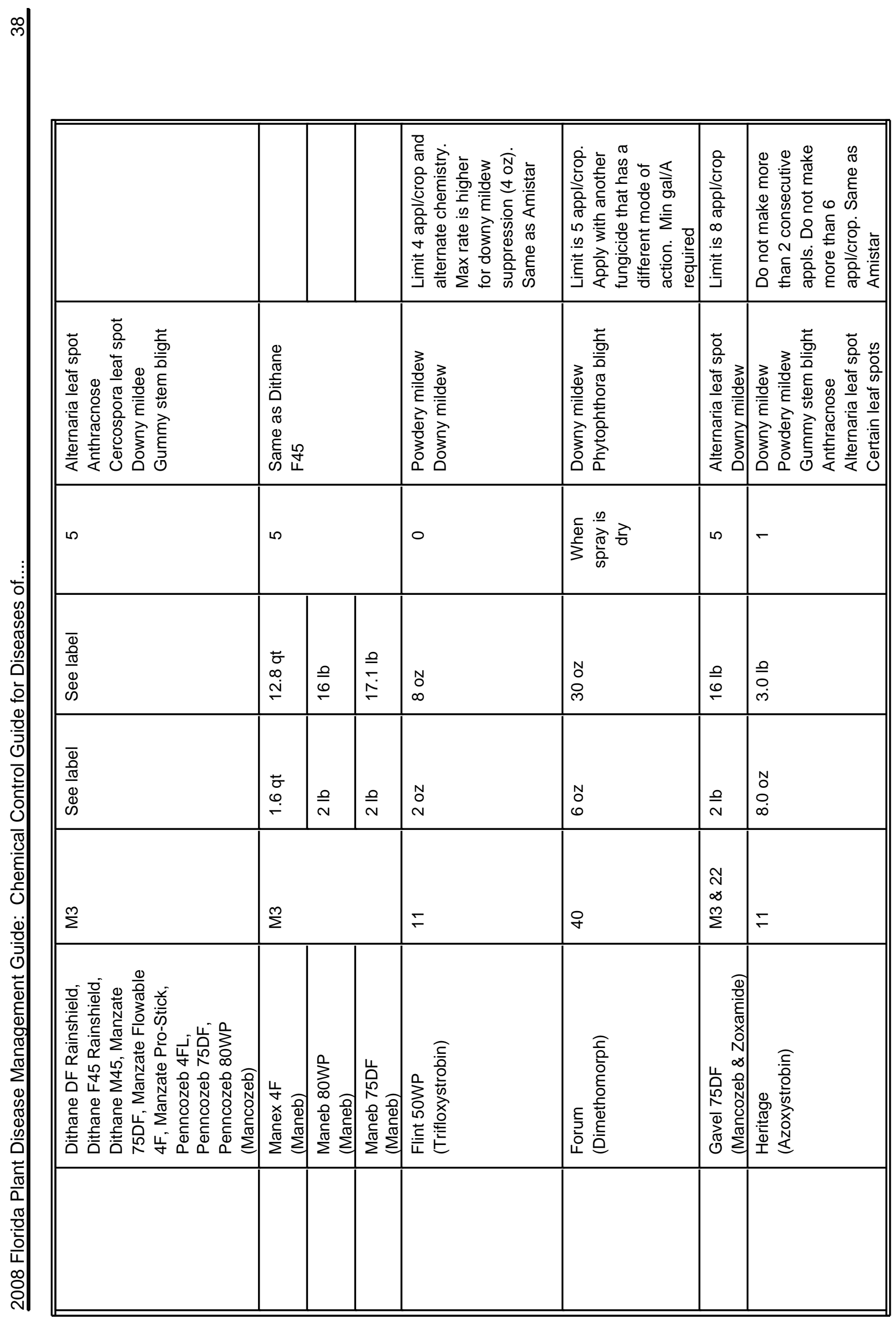




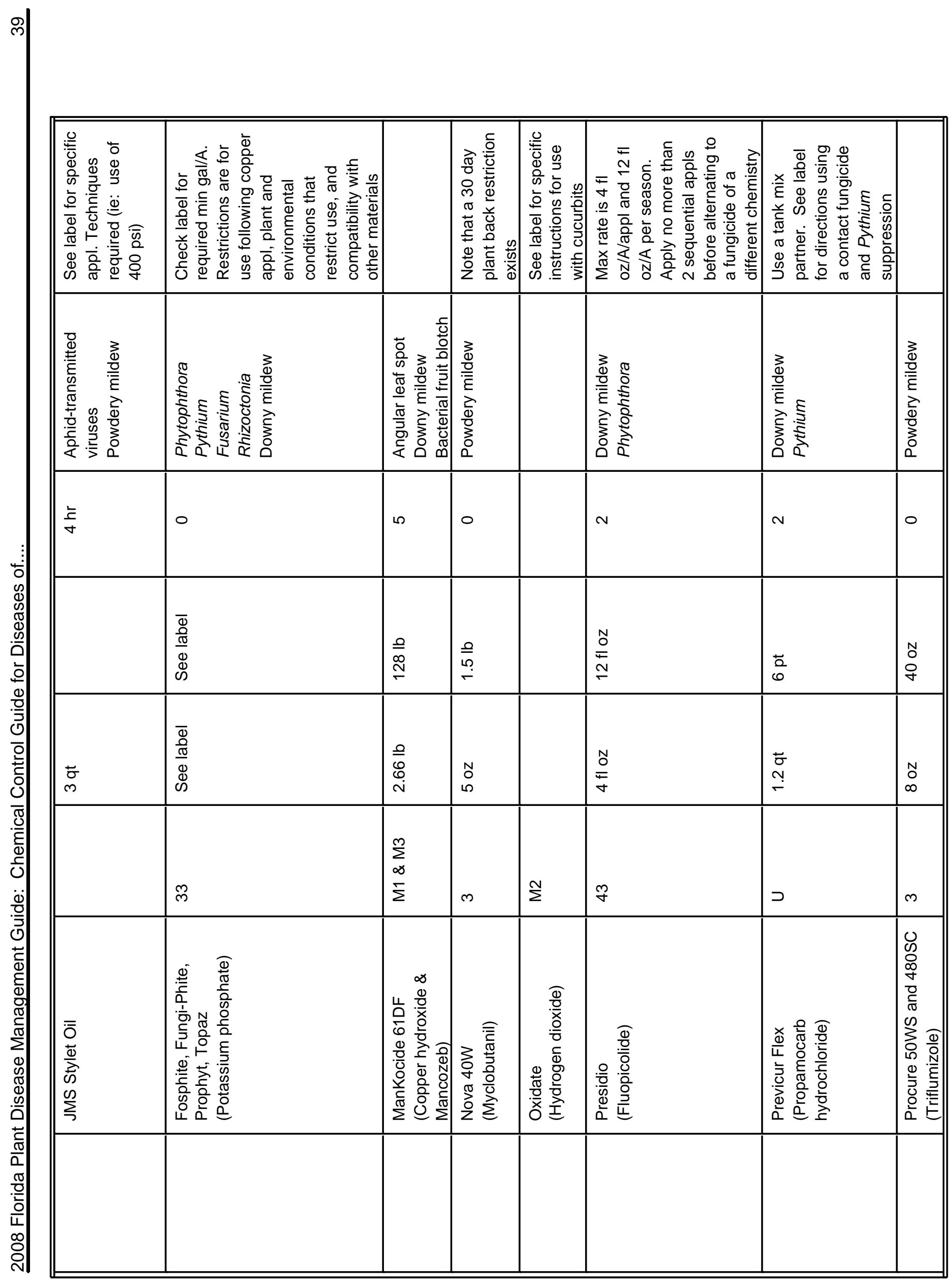




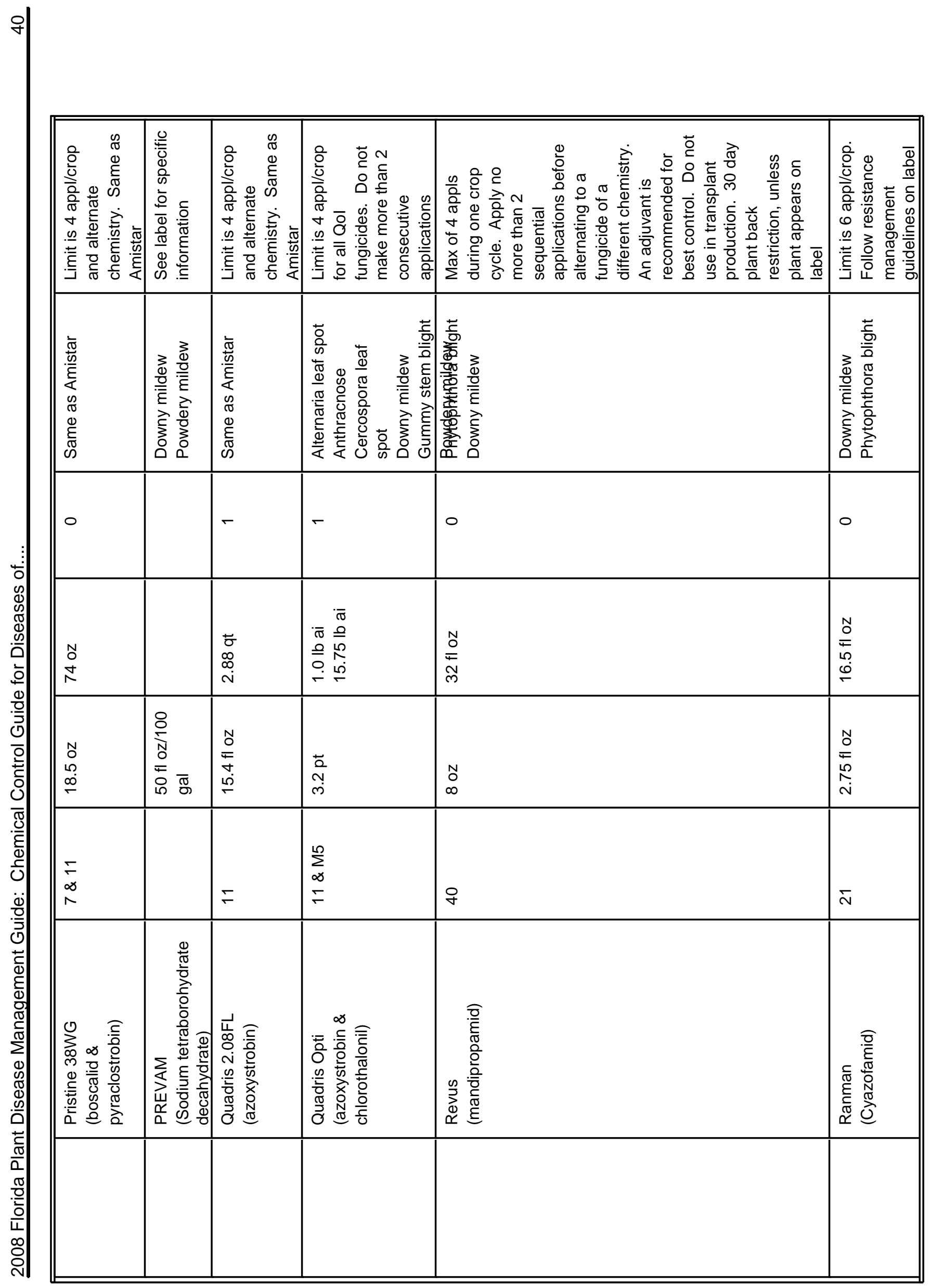




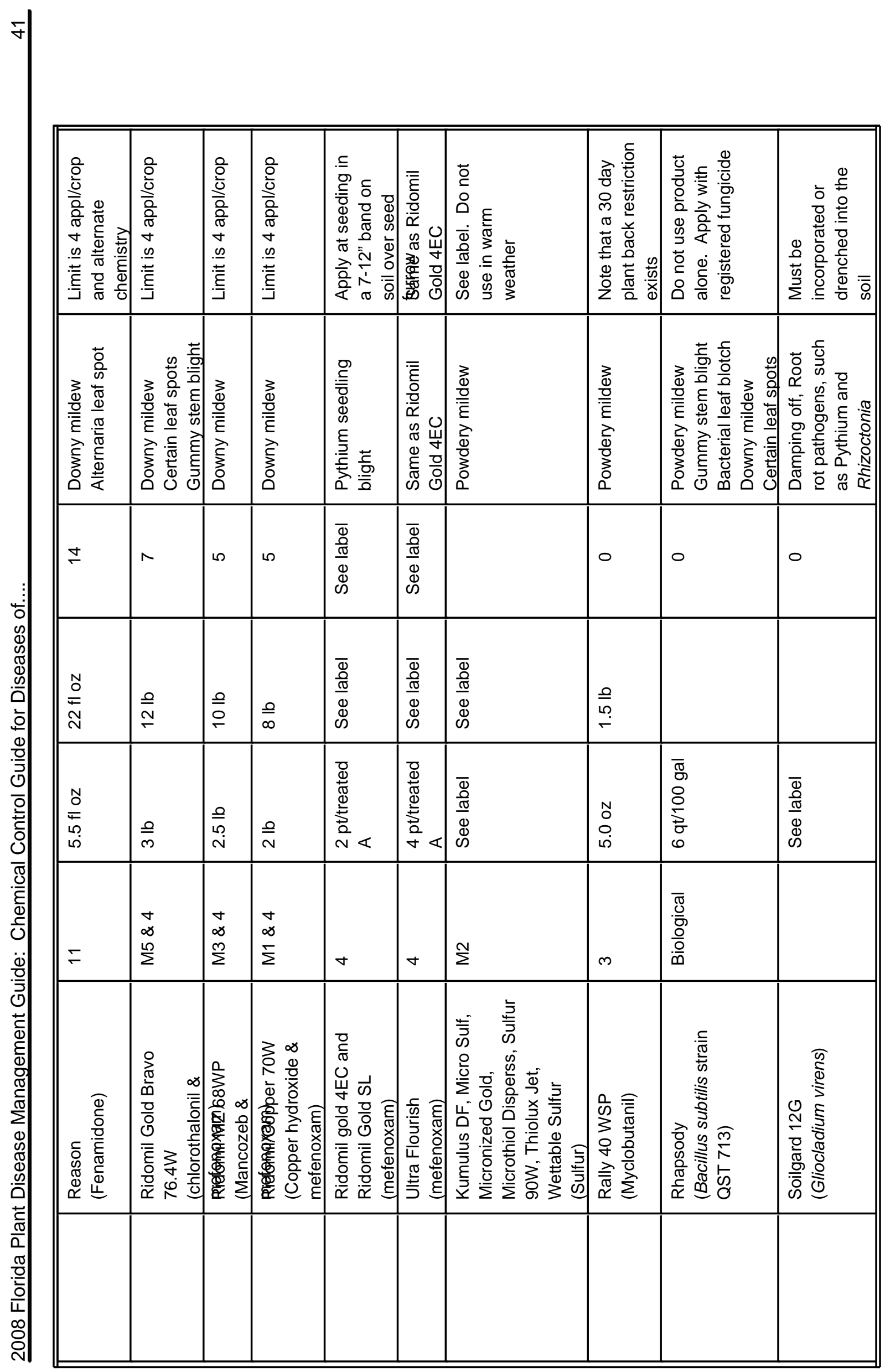




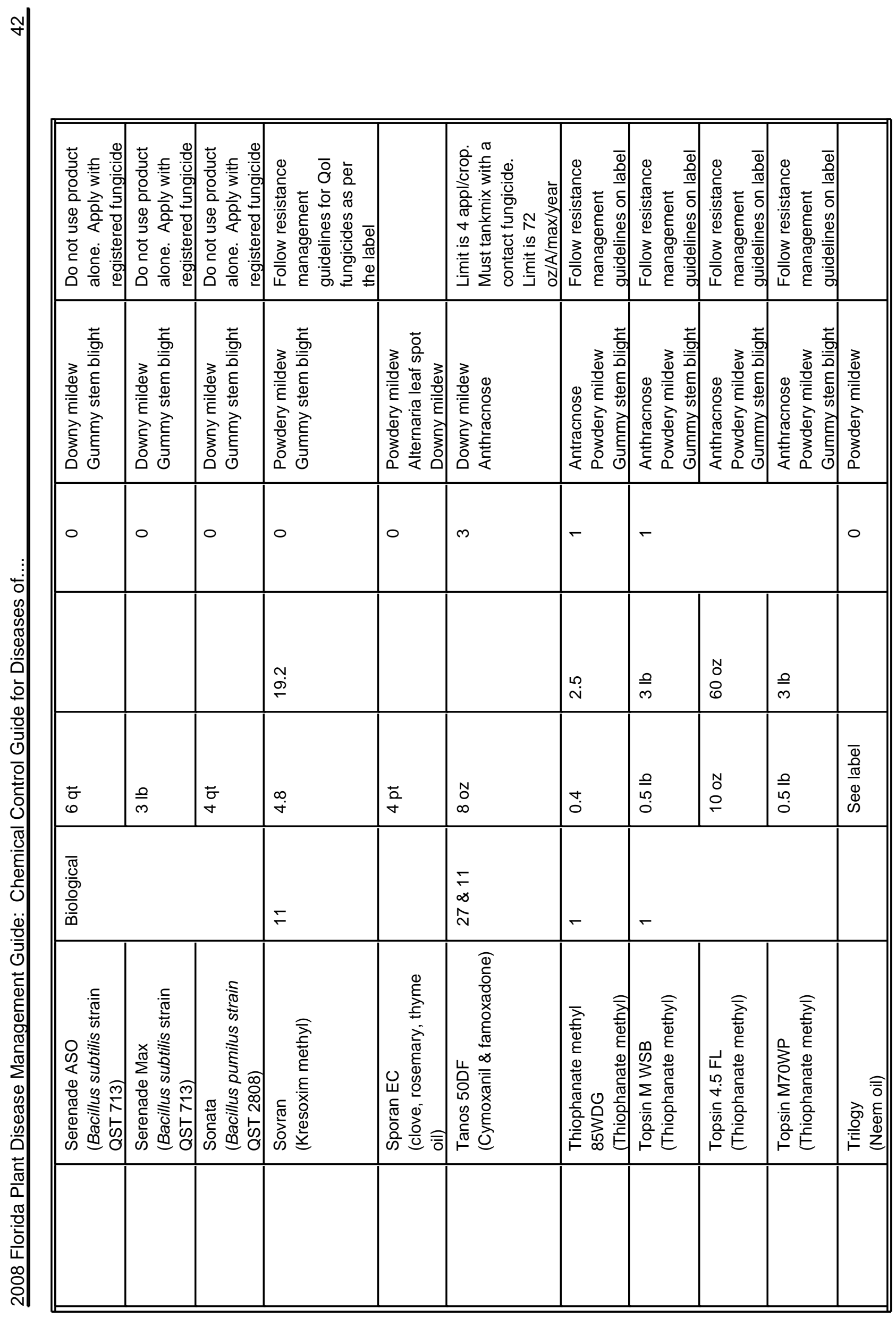




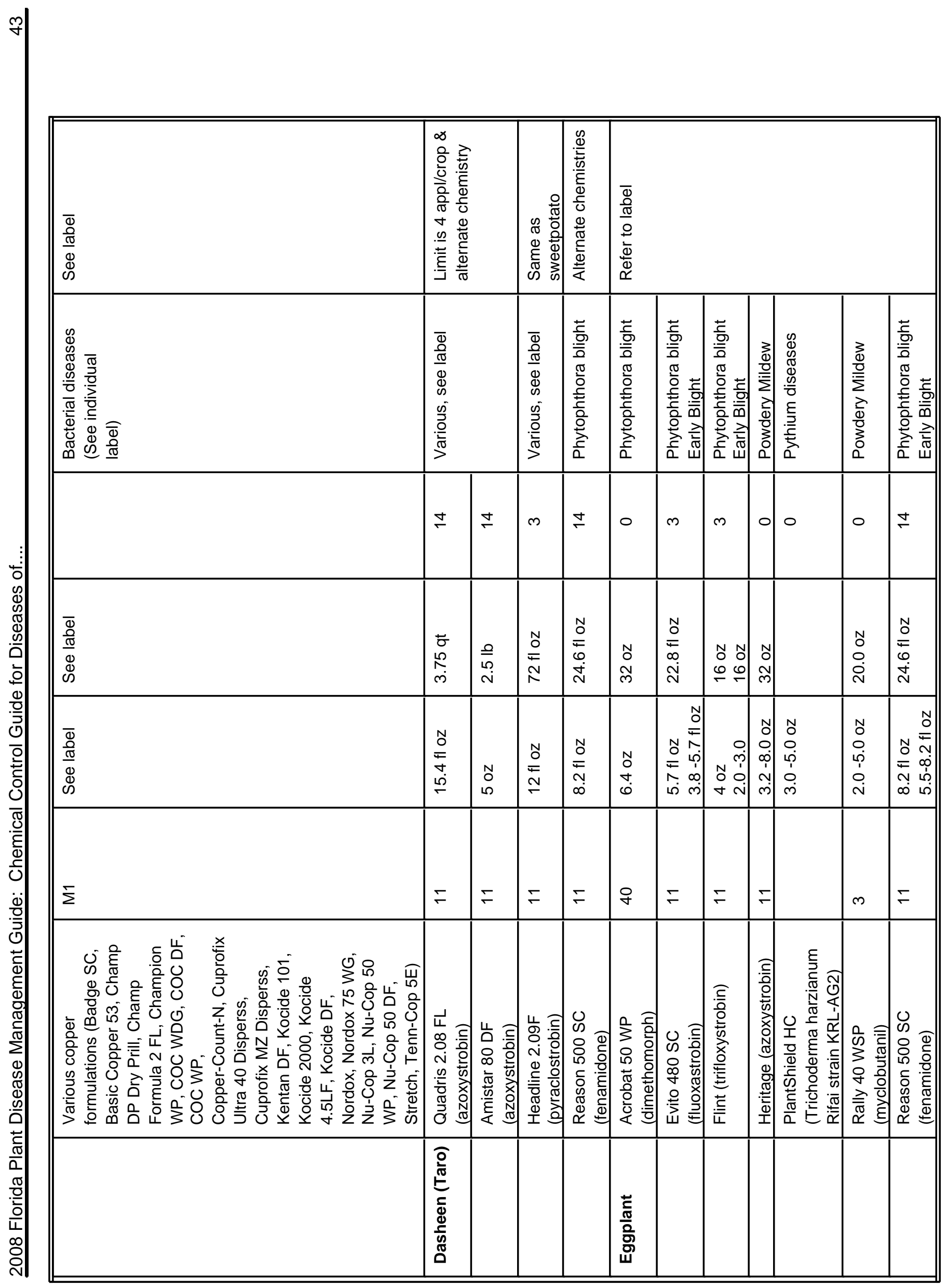




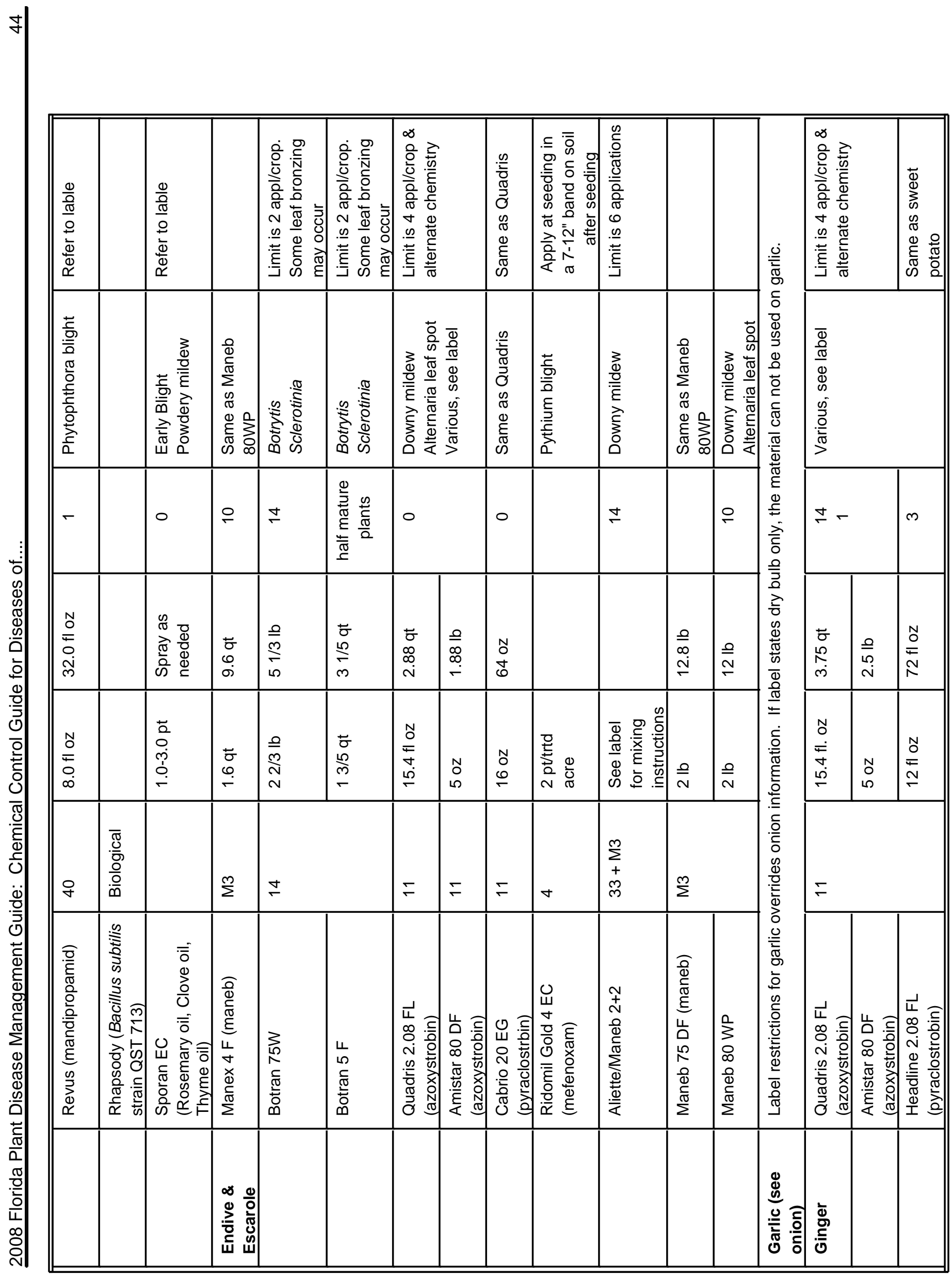




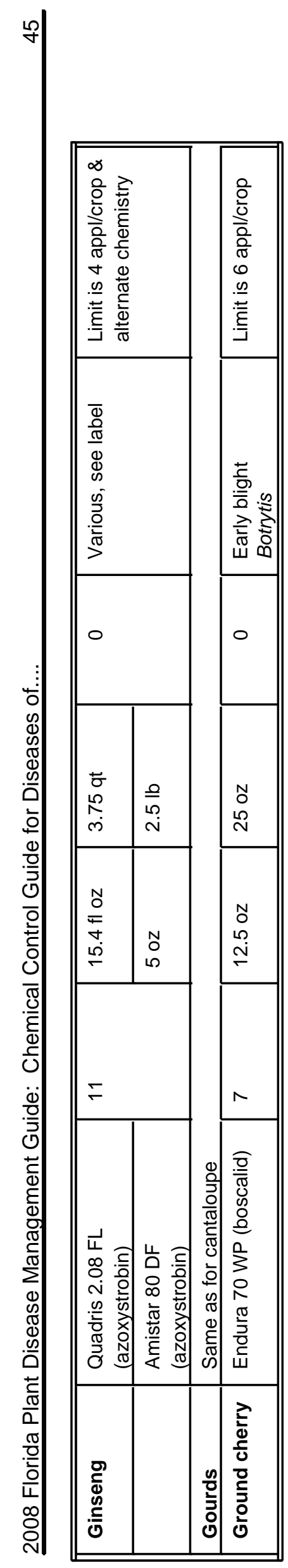




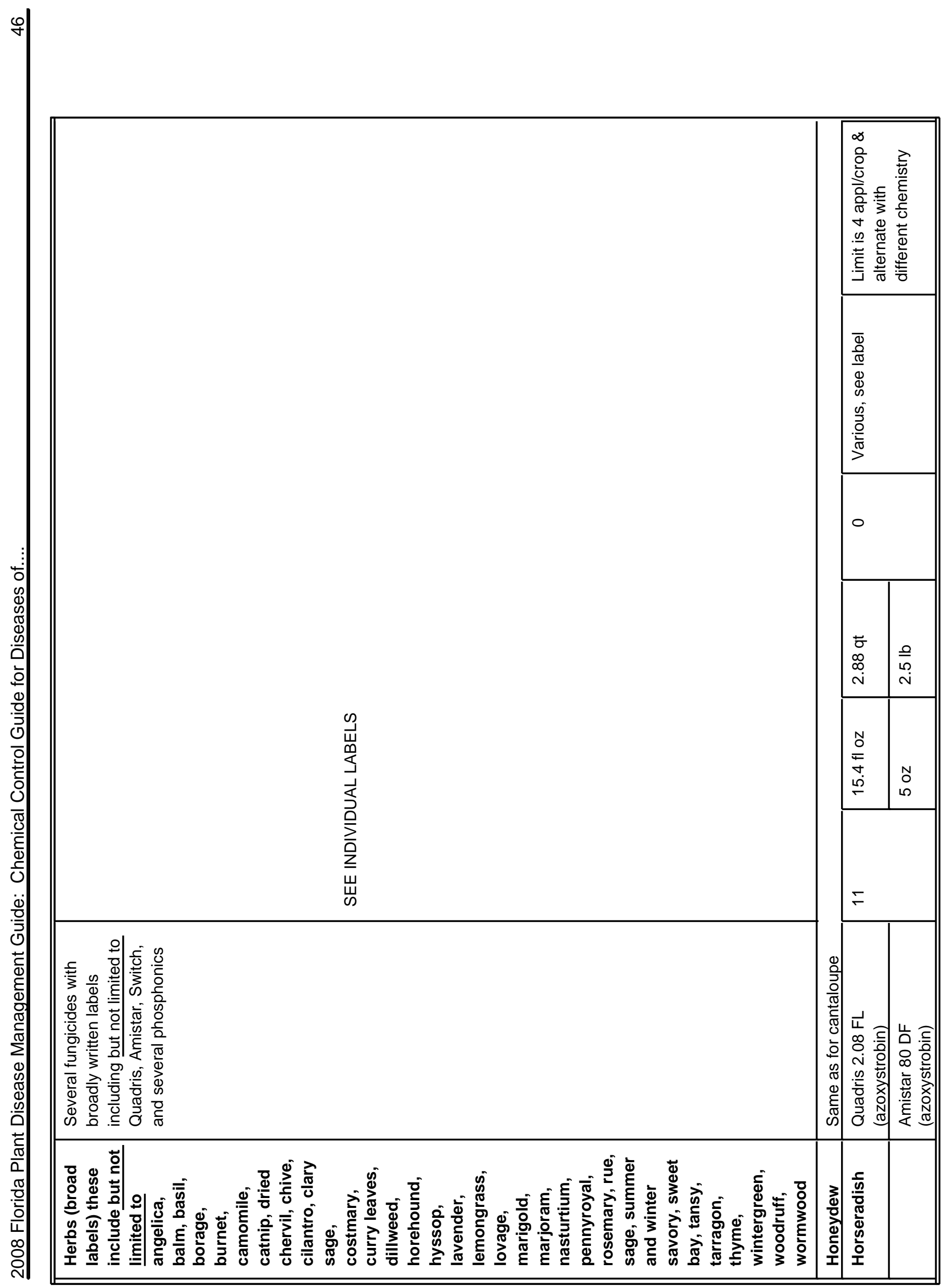




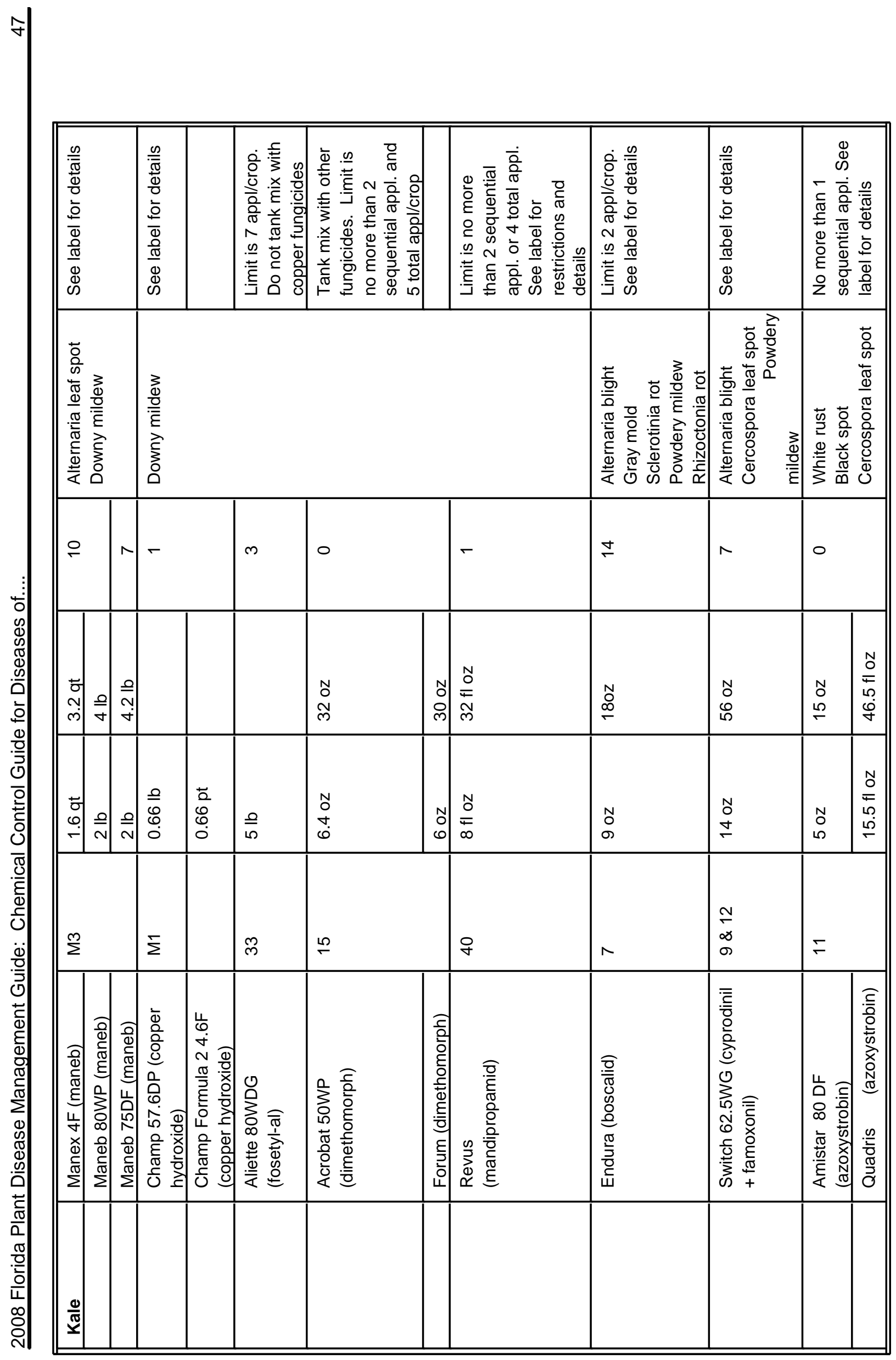




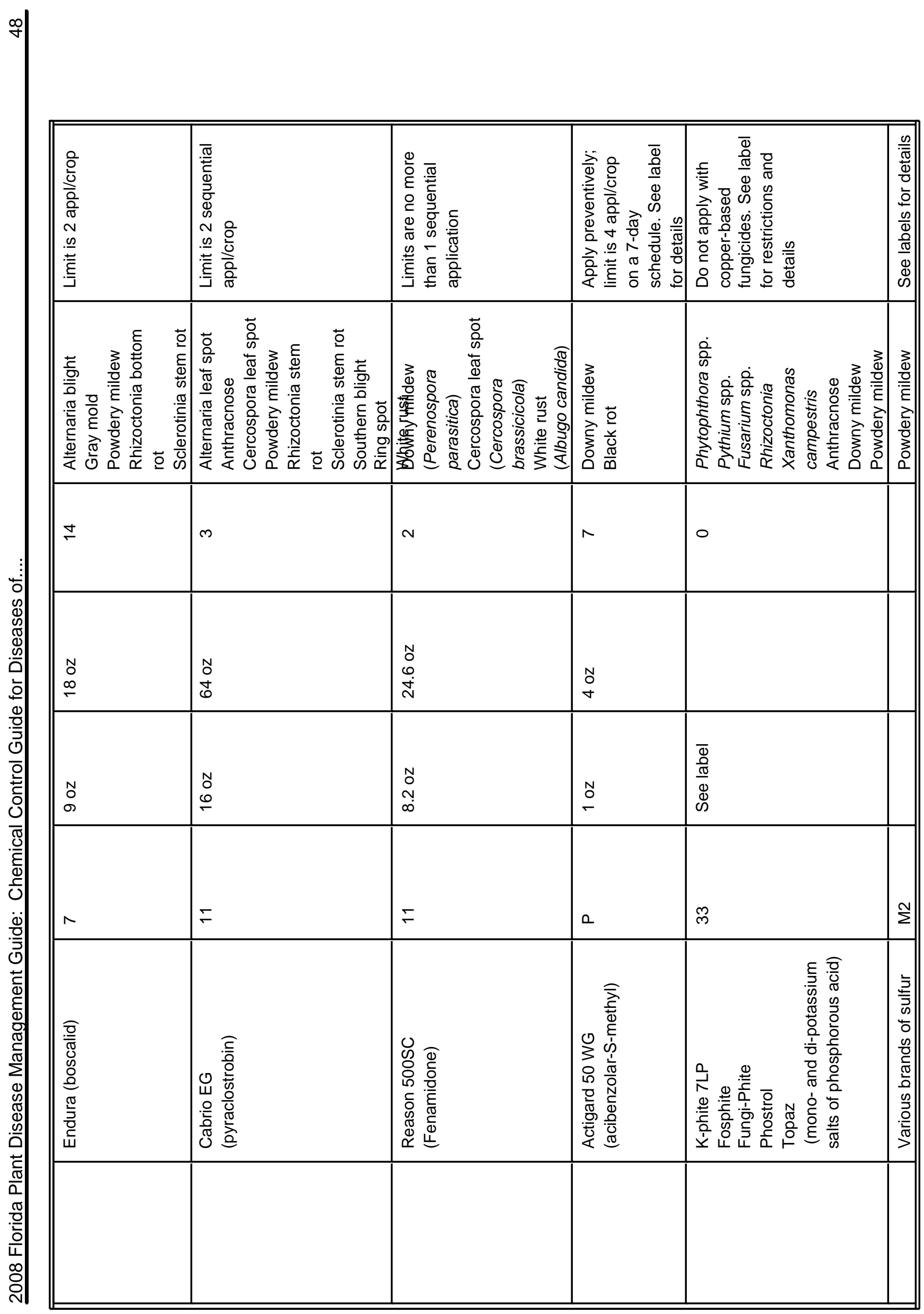




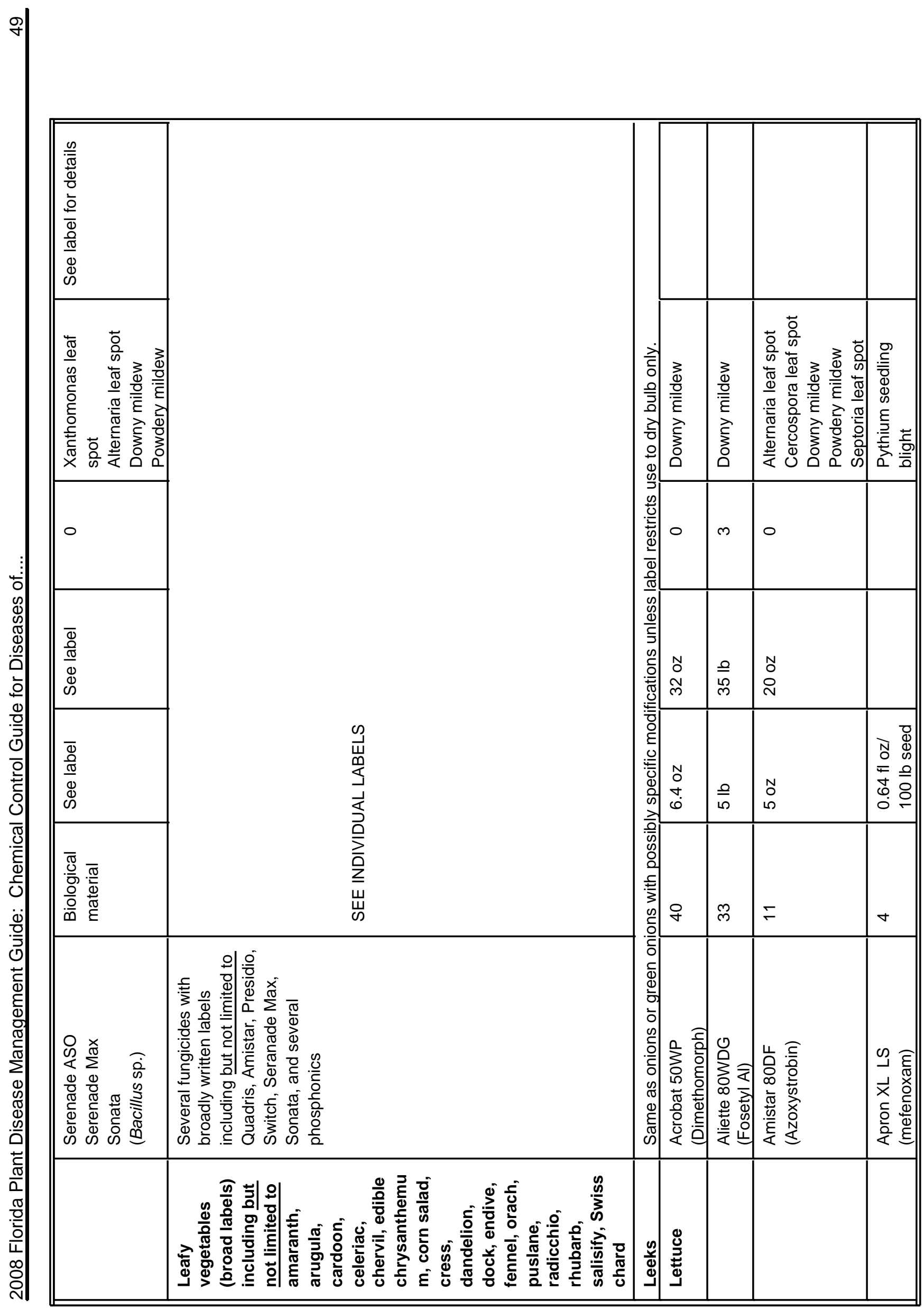




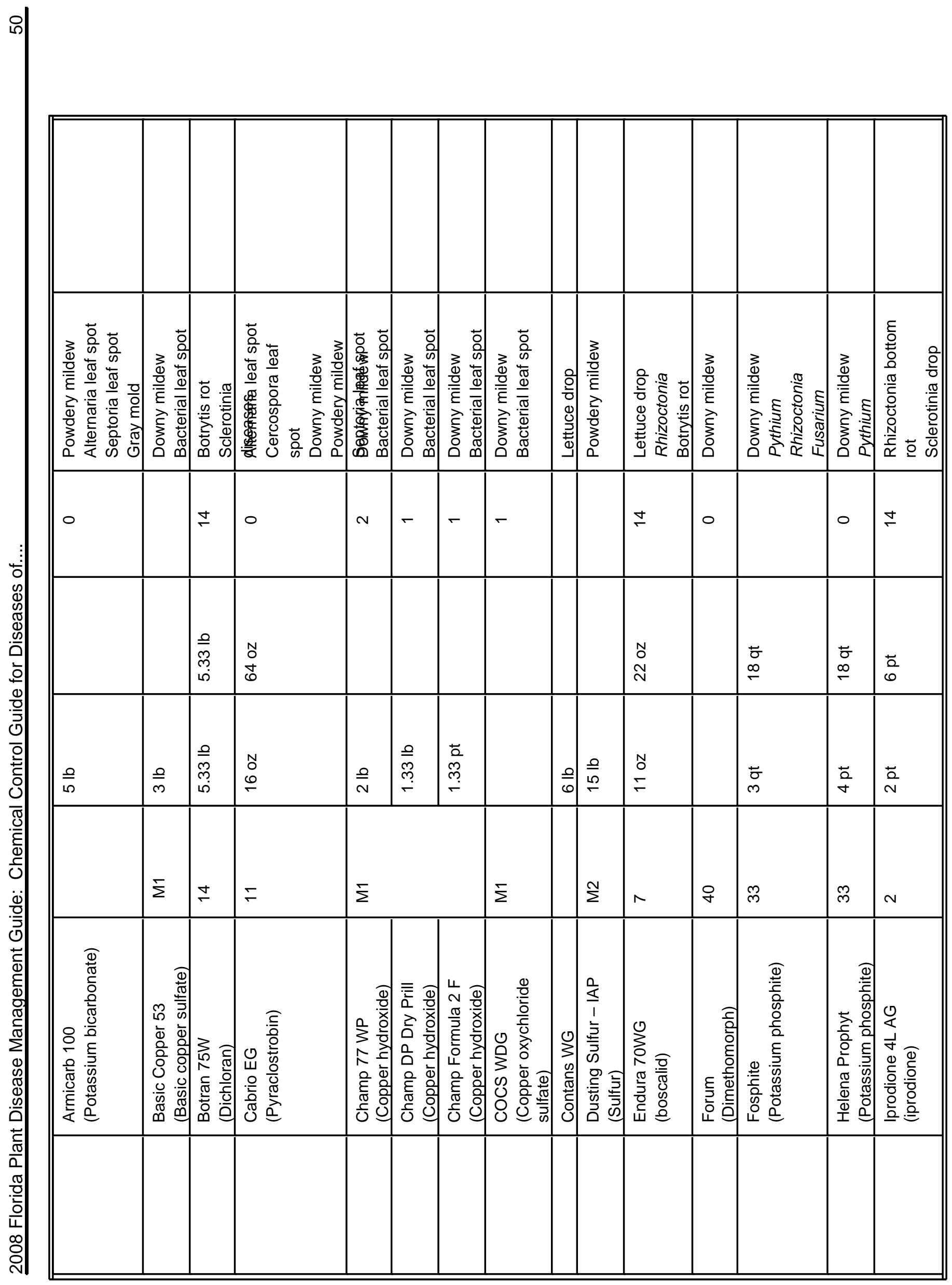




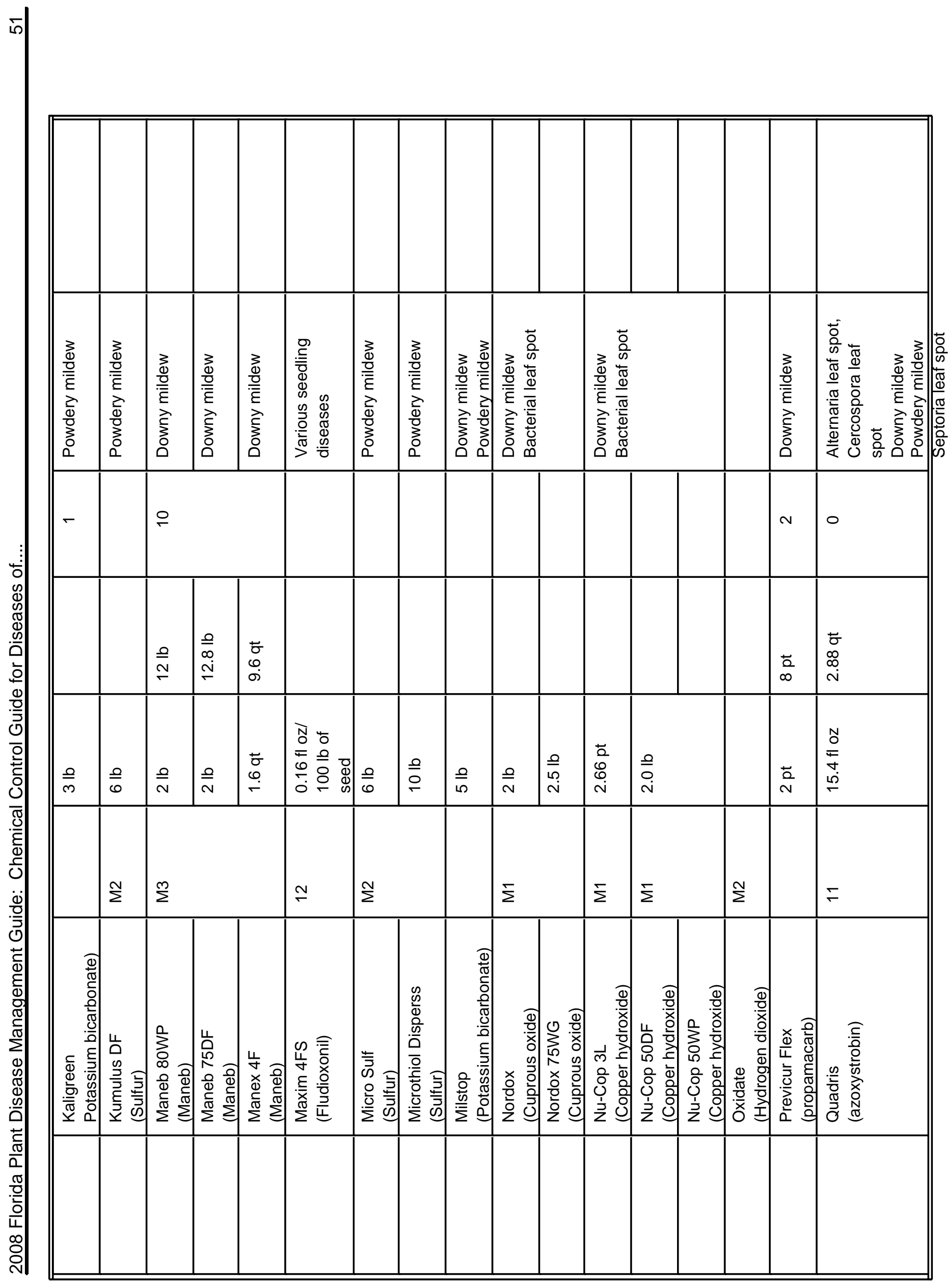




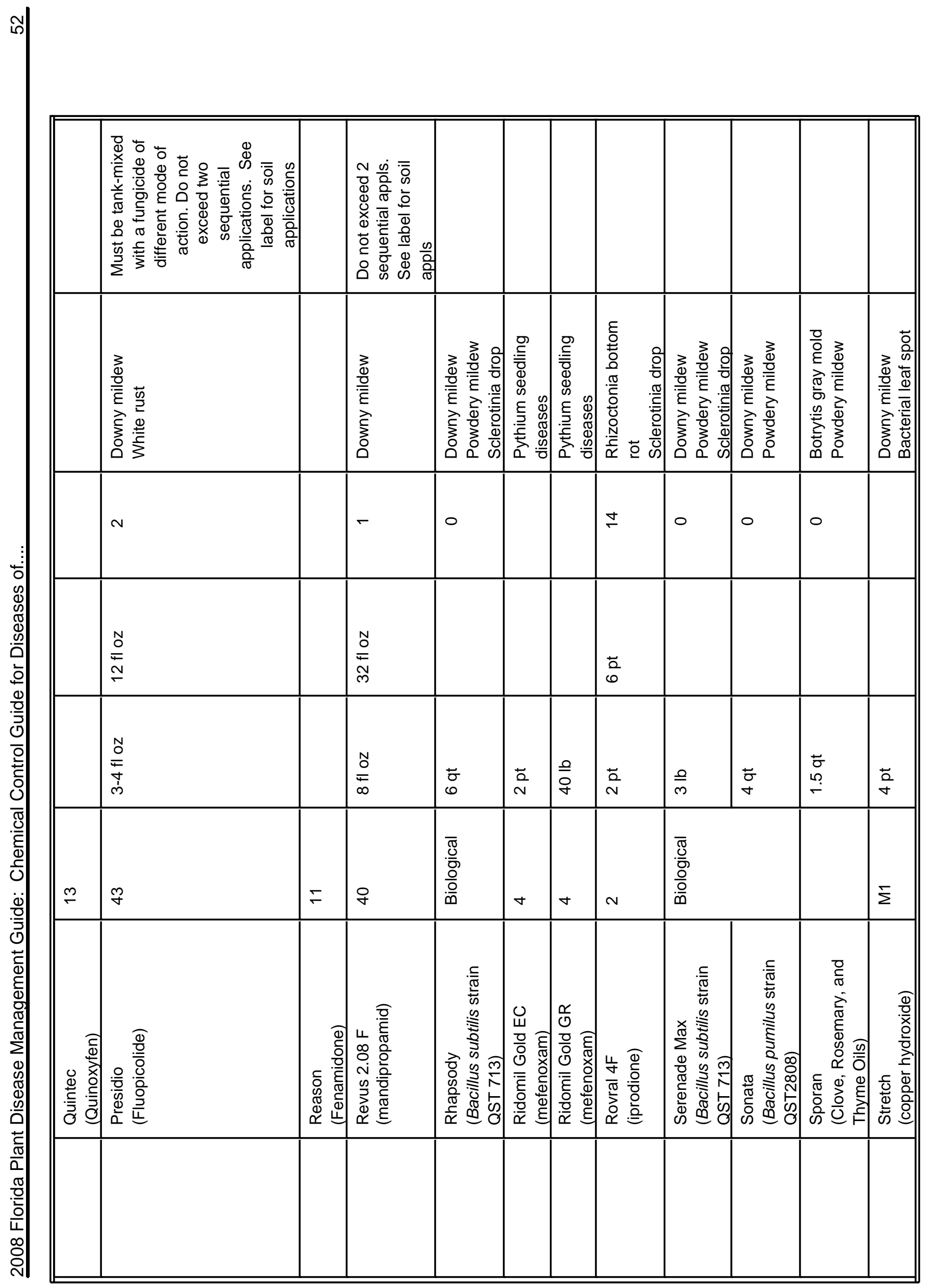




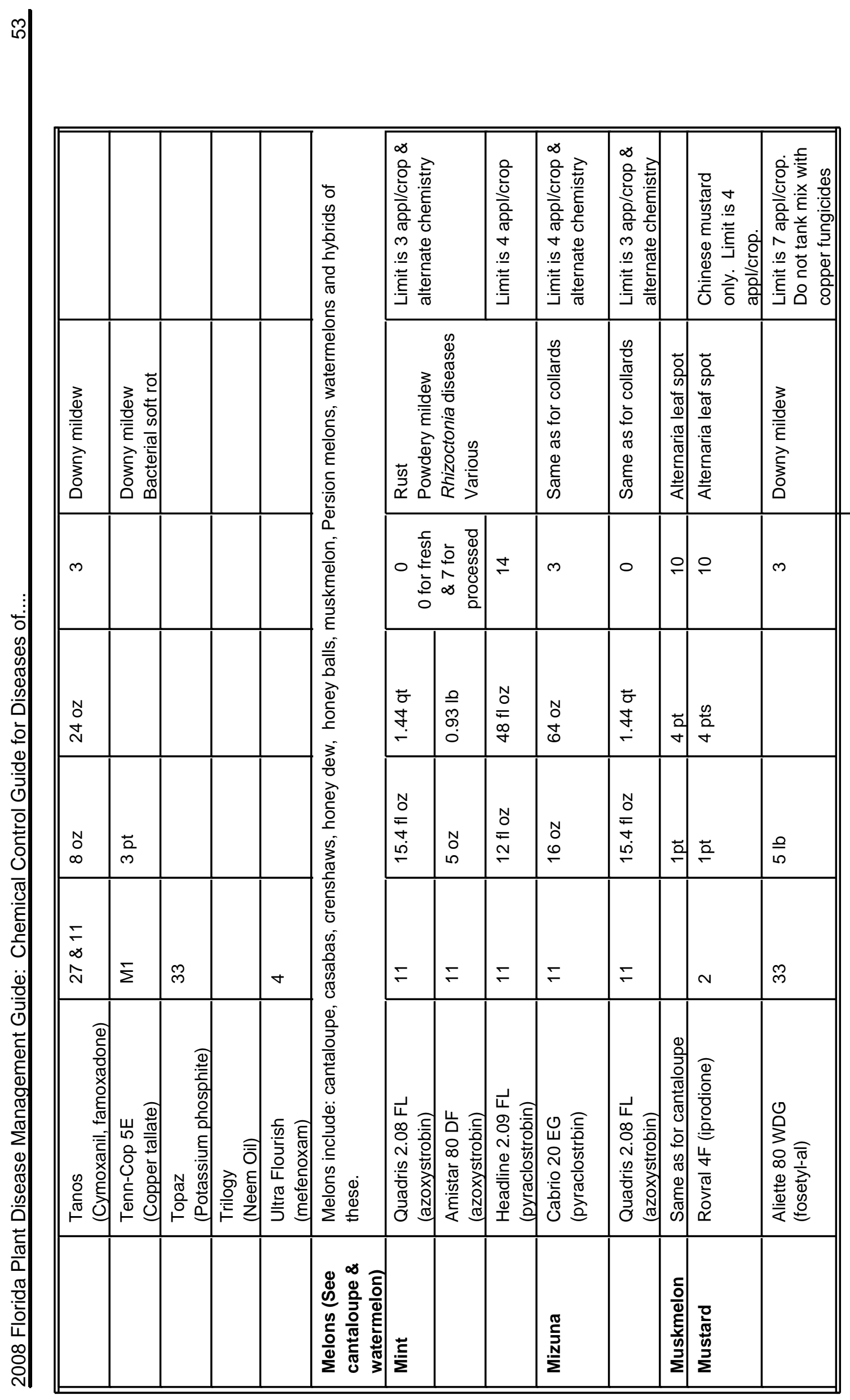




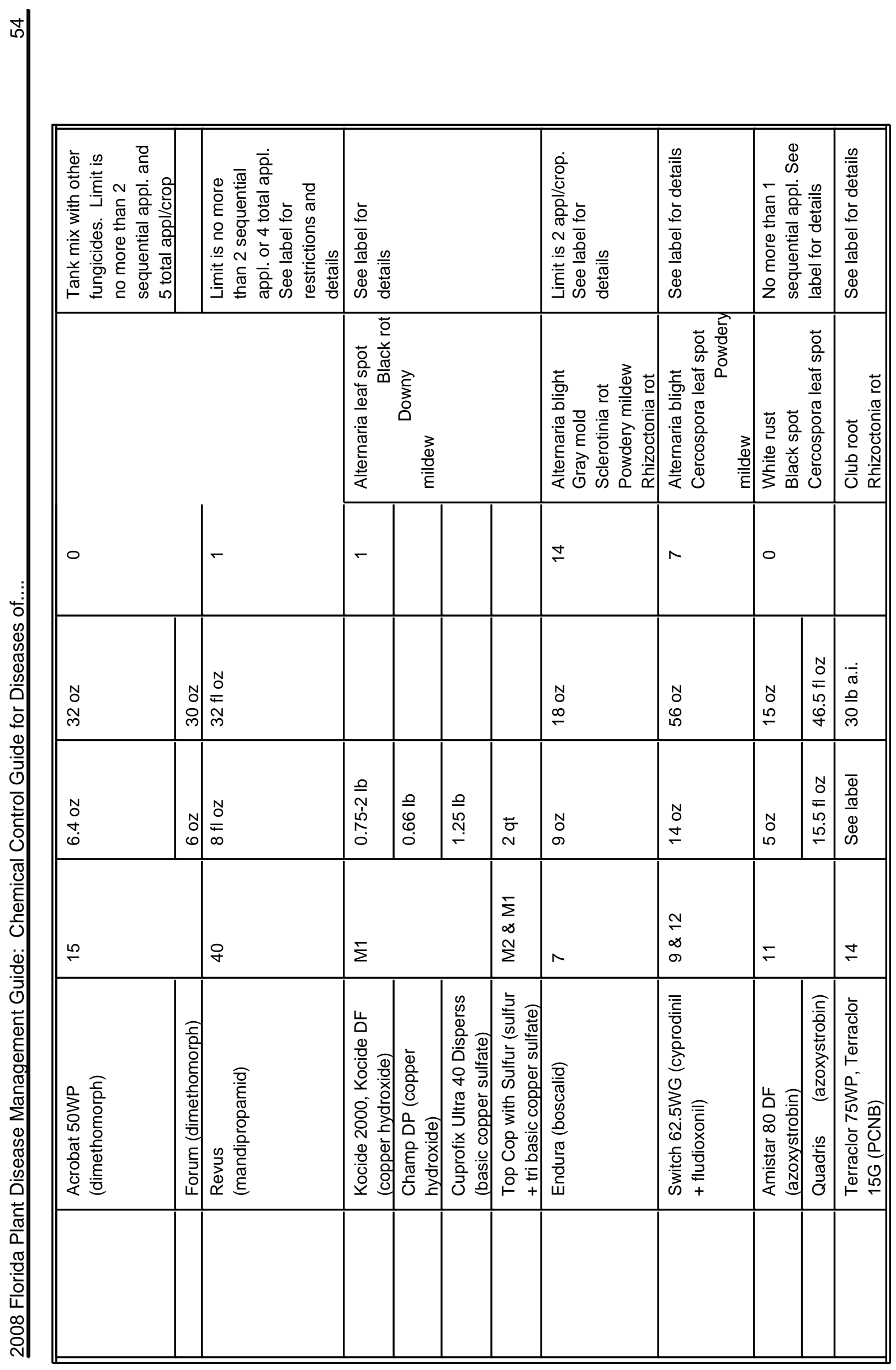




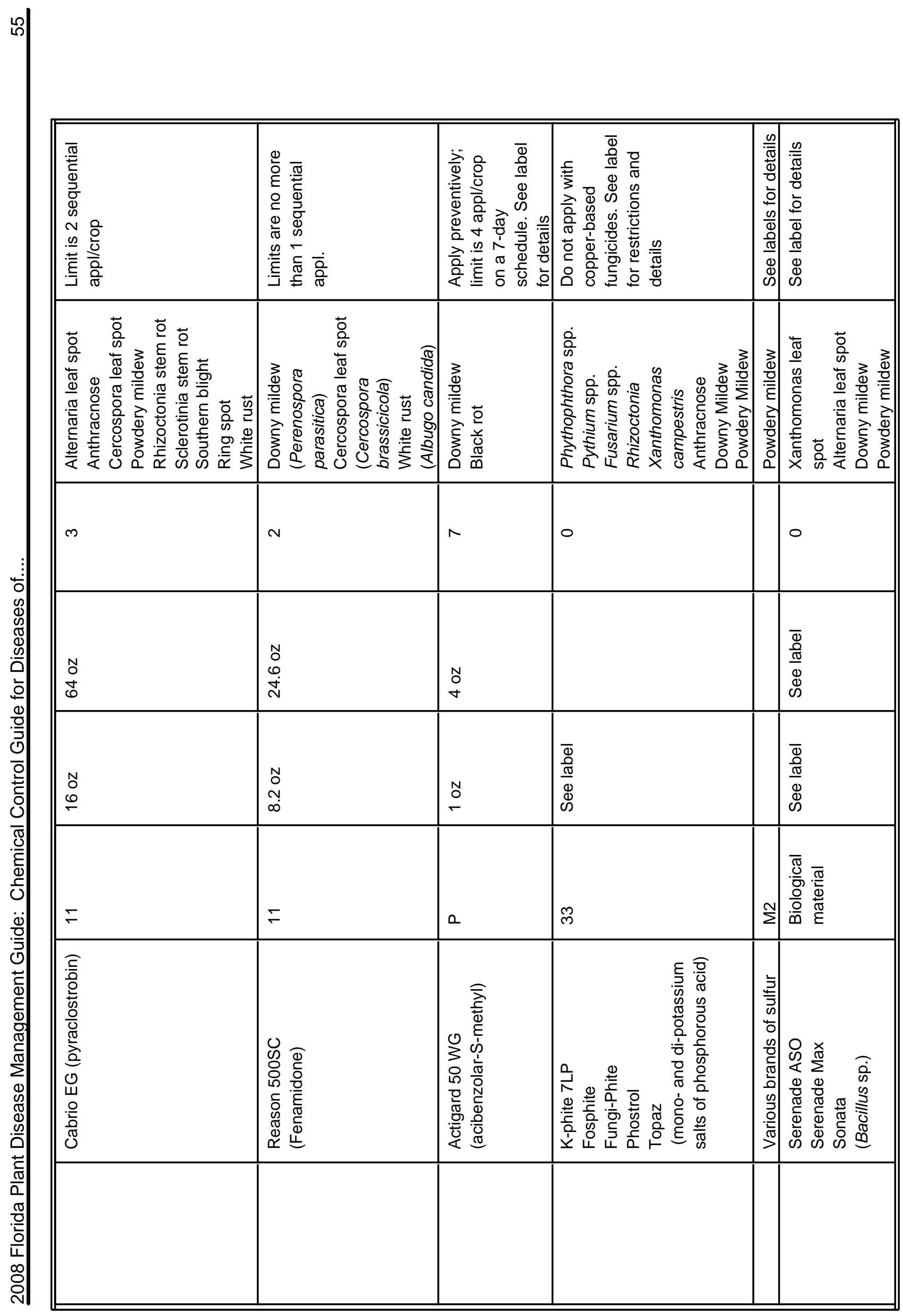




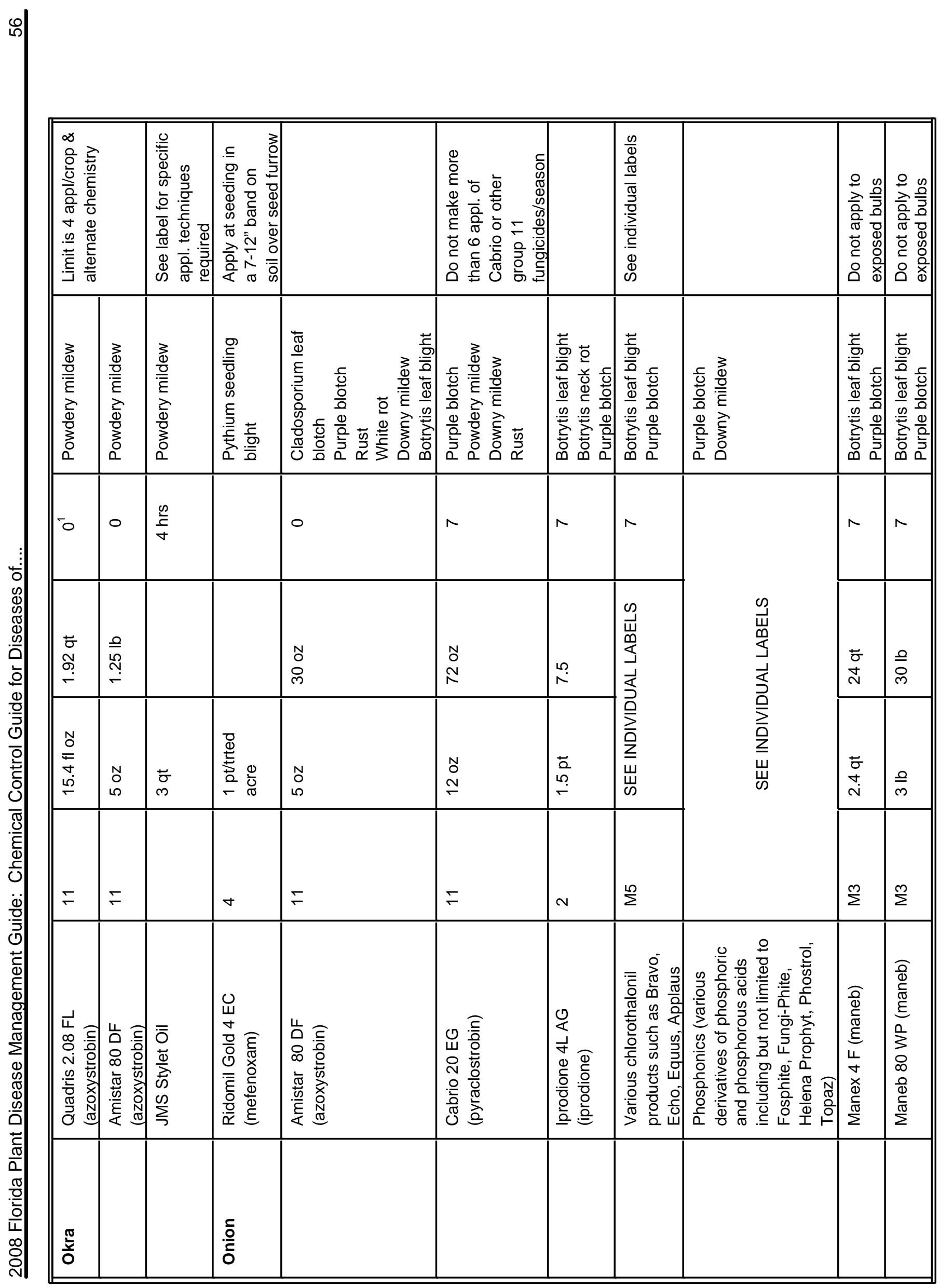




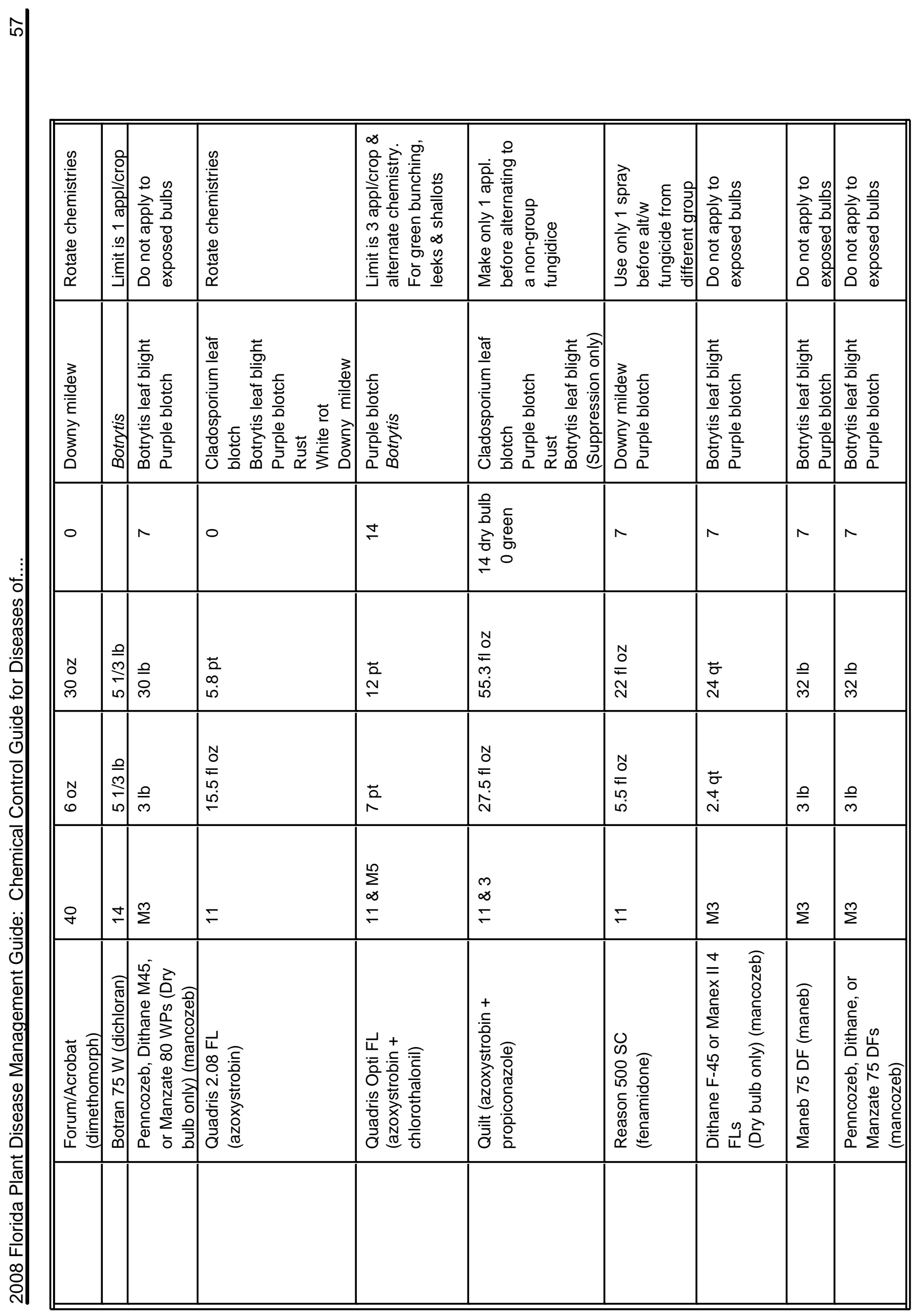




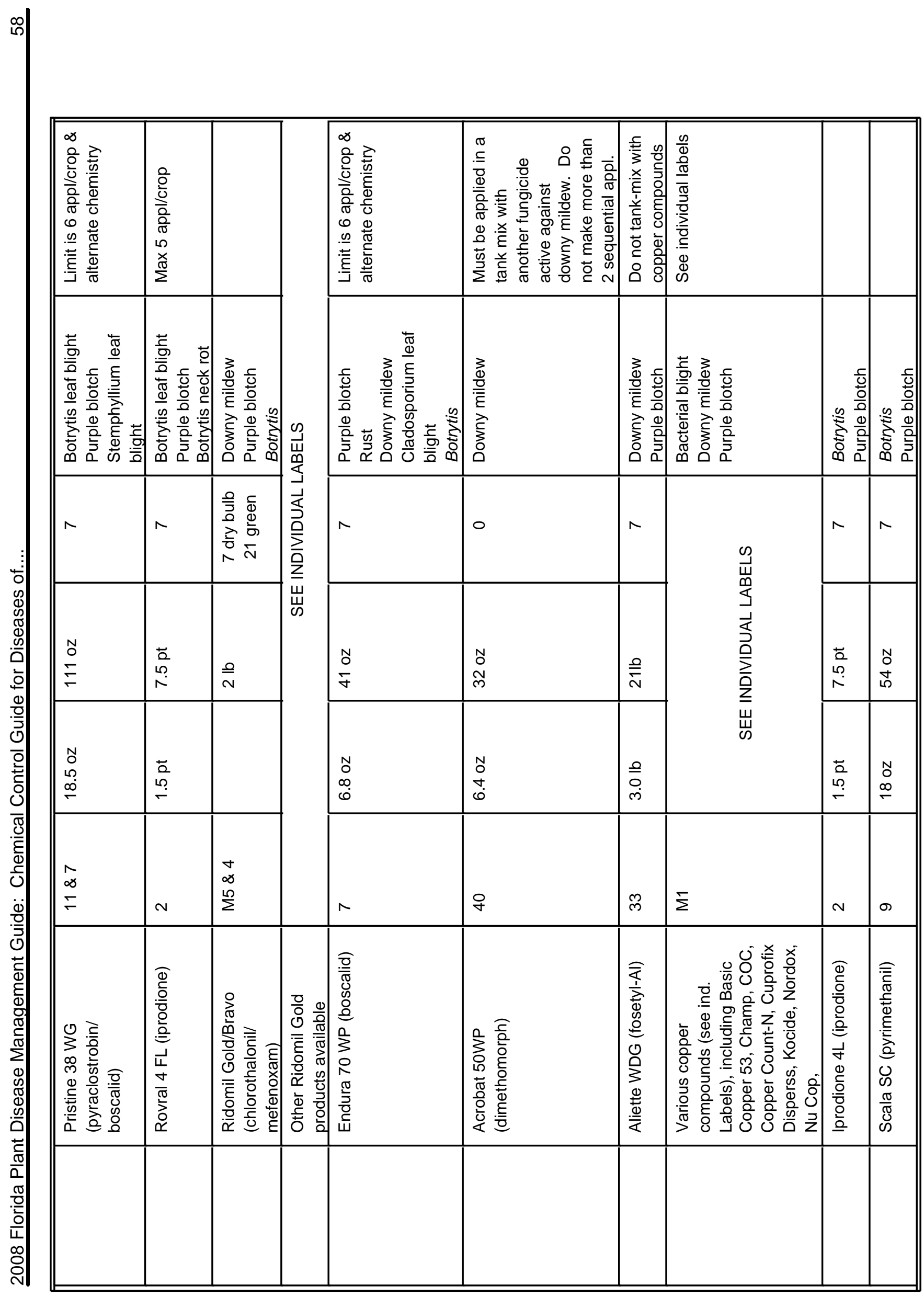




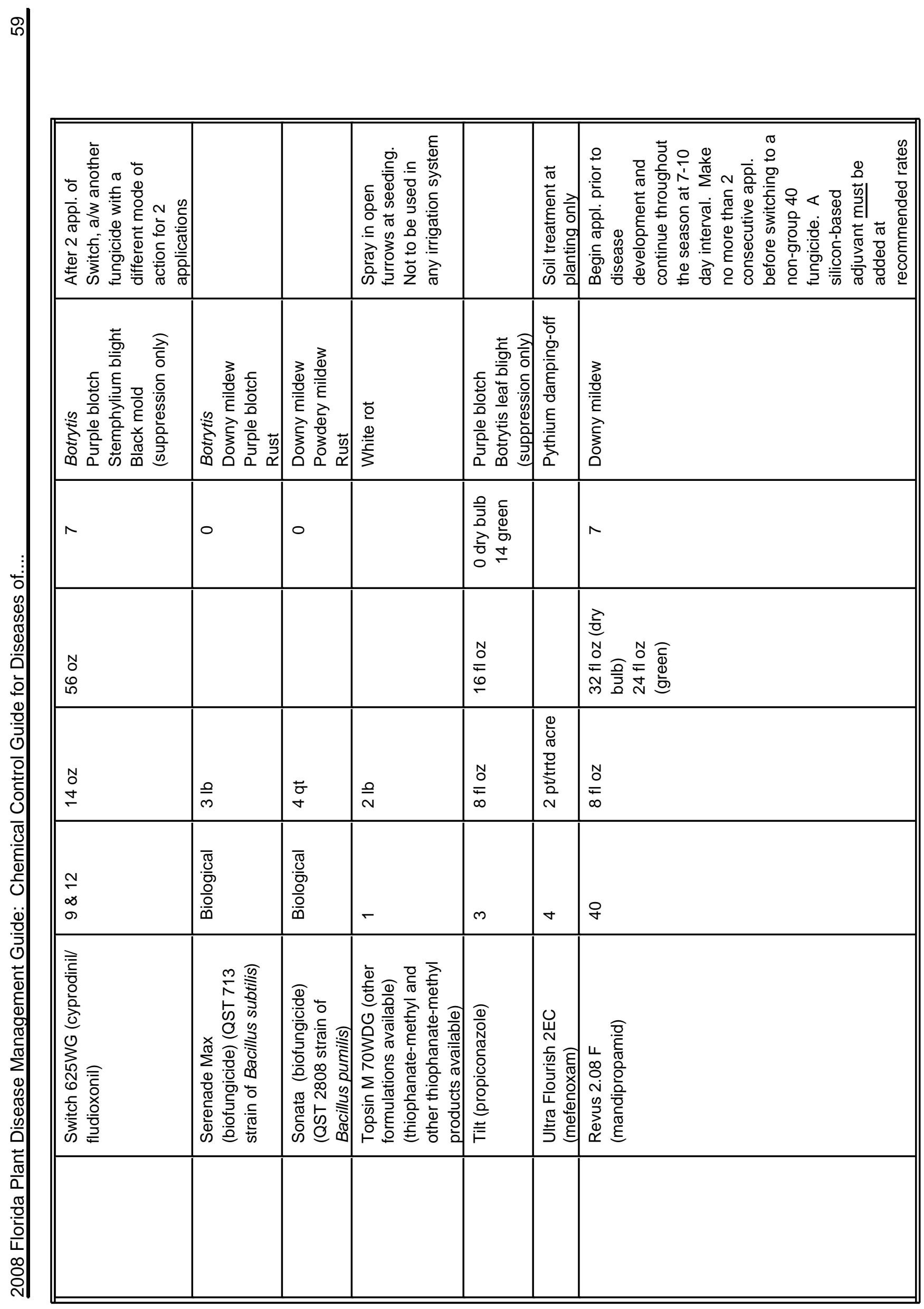




\begin{tabular}{|c|c|c|c|c|c|c|c|c|c|c|c|c|}
\hline 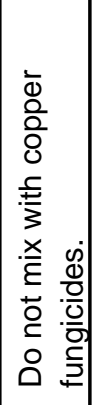 & 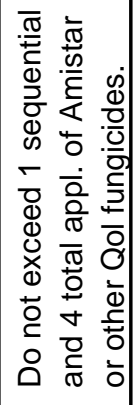 & 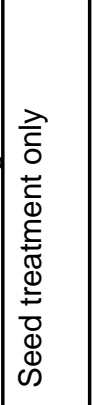 & & 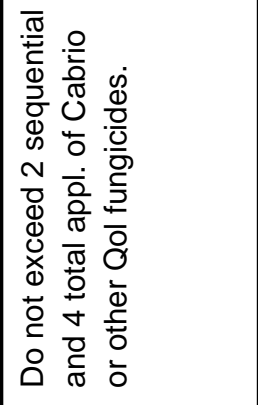 & 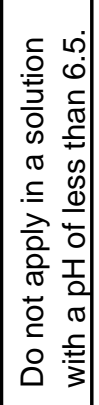 & 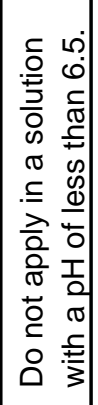 & 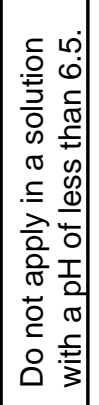 & 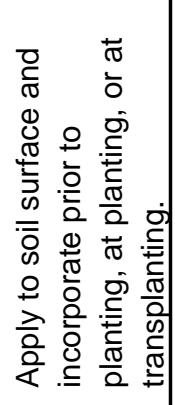 & & & 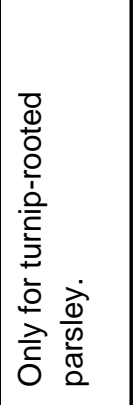 & 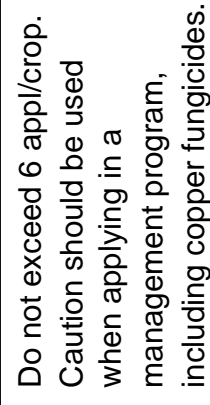 \\
\hline 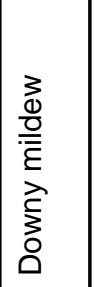 & 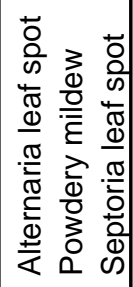 & 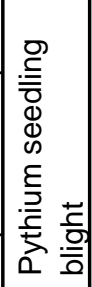 & & 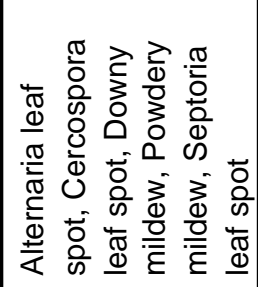 & 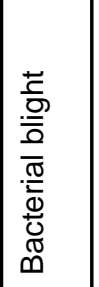 & 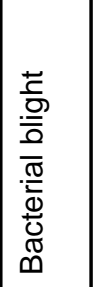 & 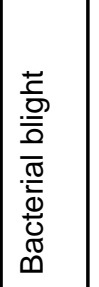 & 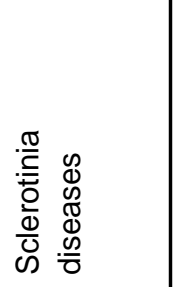 & 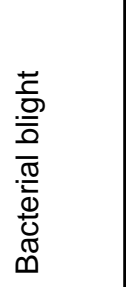 & 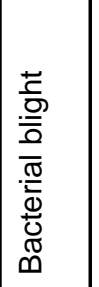 & 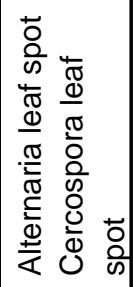 & 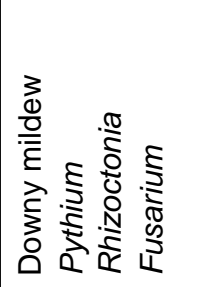 \\
\hline m & 0 & & & 0 & $\sim$ & - & - & & & & $\wedge$ & \\
\hline$\stackrel{\rho}{\stackrel{\rho}{\nu}}$ & $\begin{array}{l}N \\
\text { O } \\
\text { N }\end{array}$ & & & $\begin{array}{l}N \\
O \\
\mathbb{U}\end{array}$ & & & & & & & $\begin{array}{l}\stackrel{N}{\circ} \\
\stackrel{N}{\simeq}\end{array}$ & $\begin{array}{l}\overrightarrow{0} \\
\infty \\
-\end{array}$ \\
\hline$\frac{0}{10}$ & $\begin{array}{l}N \\
0 \\
0\end{array}$ & 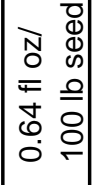 & & $\begin{array}{l}N \\
O \\
0\end{array}$ & $\stackrel{\rho}{\sim}$ & $\stackrel{\underline{\mathrm{N}}}{\sim}$ & $\stackrel{\mathrm{a}}{\mathrm{N}}$ & $\frac{0}{6}$ & $\begin{array}{l}\vec{v} \\
\text { ल }\end{array}$ & $\stackrel{\rho}{\vec{\forall}}$ & $\begin{array}{l}\text { No } \\
\text { M }\end{array}$ & $\begin{array}{l}+\overrightarrow{0} \\
\text { m }\end{array}$ \\
\hline m & $\mp$ & $\nabla$ & $\Sigma$ & $\mp$ & $\Sigma$ & $\Sigma$ & $\Sigma$ & & $\Sigma$ & $\Sigma$ & $\mp$ & ల్ల \\
\hline 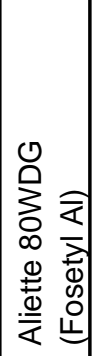 & 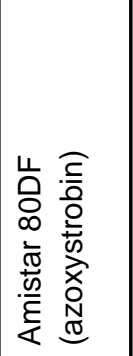 & 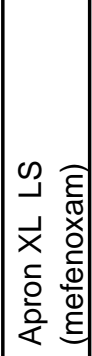 & 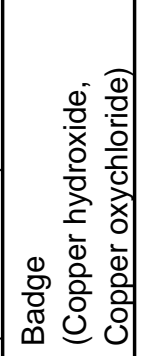 & 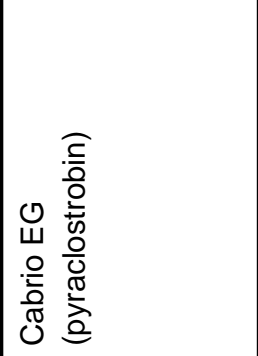 & 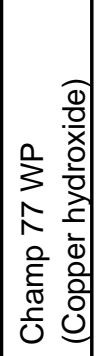 & 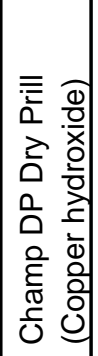 & 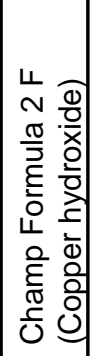 & $\begin{array}{l}0 \\
0 \\
3 \\
0 \\
\stackrel{0}{0} \\
\stackrel{0}{0} \\
0\end{array}$ & 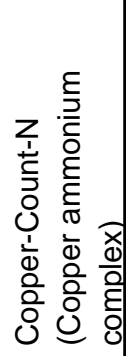 & 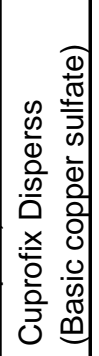 & 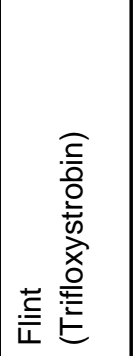 & 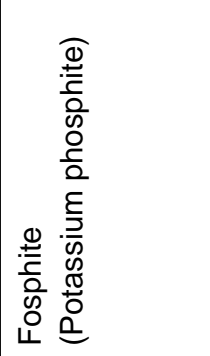 \\
\hline$\frac{\frac{\partial}{\omega}}{\frac{\pi}{\sigma 0}}$ & & & & & & & & & & & & \\
\hline
\end{tabular}




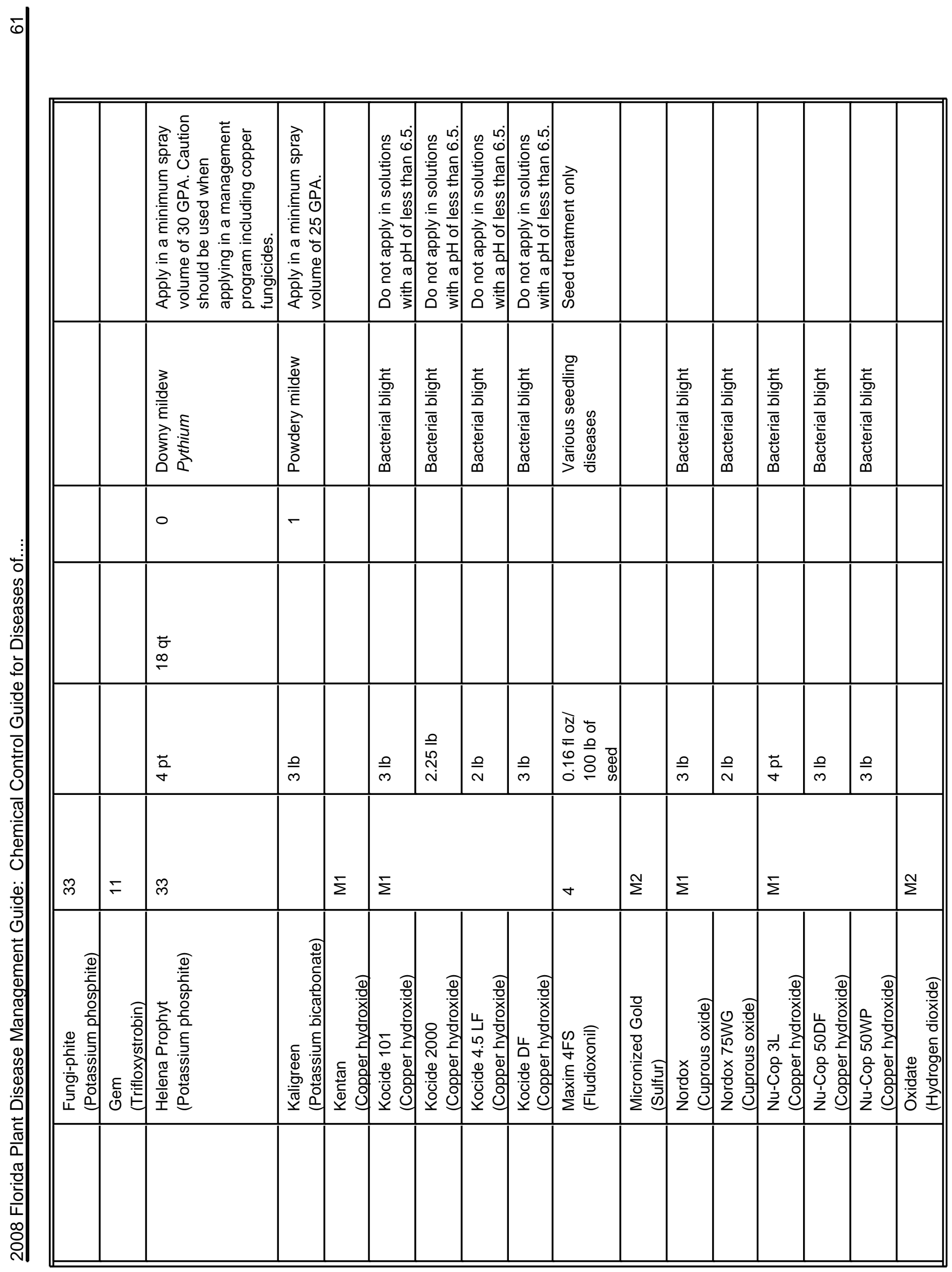




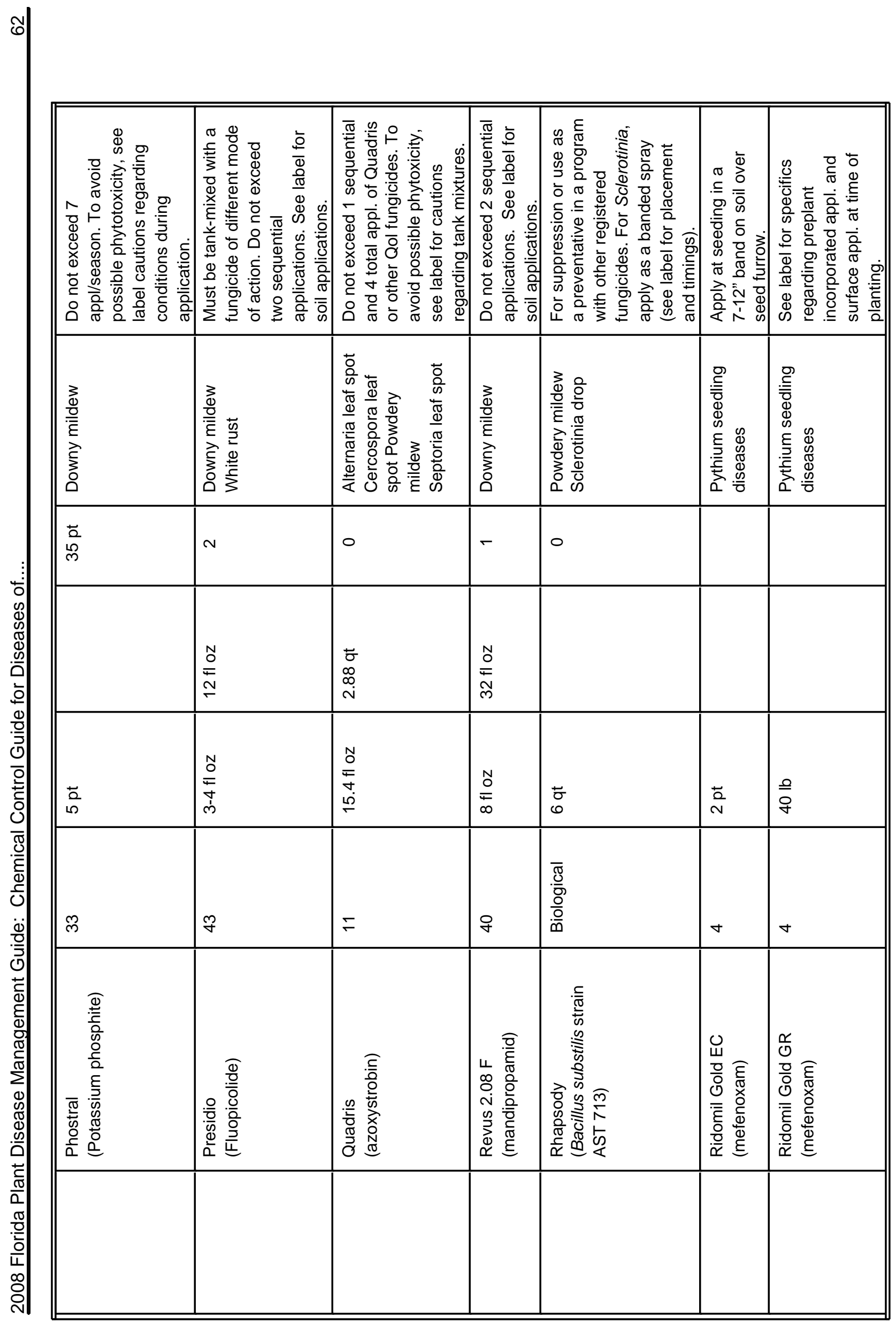




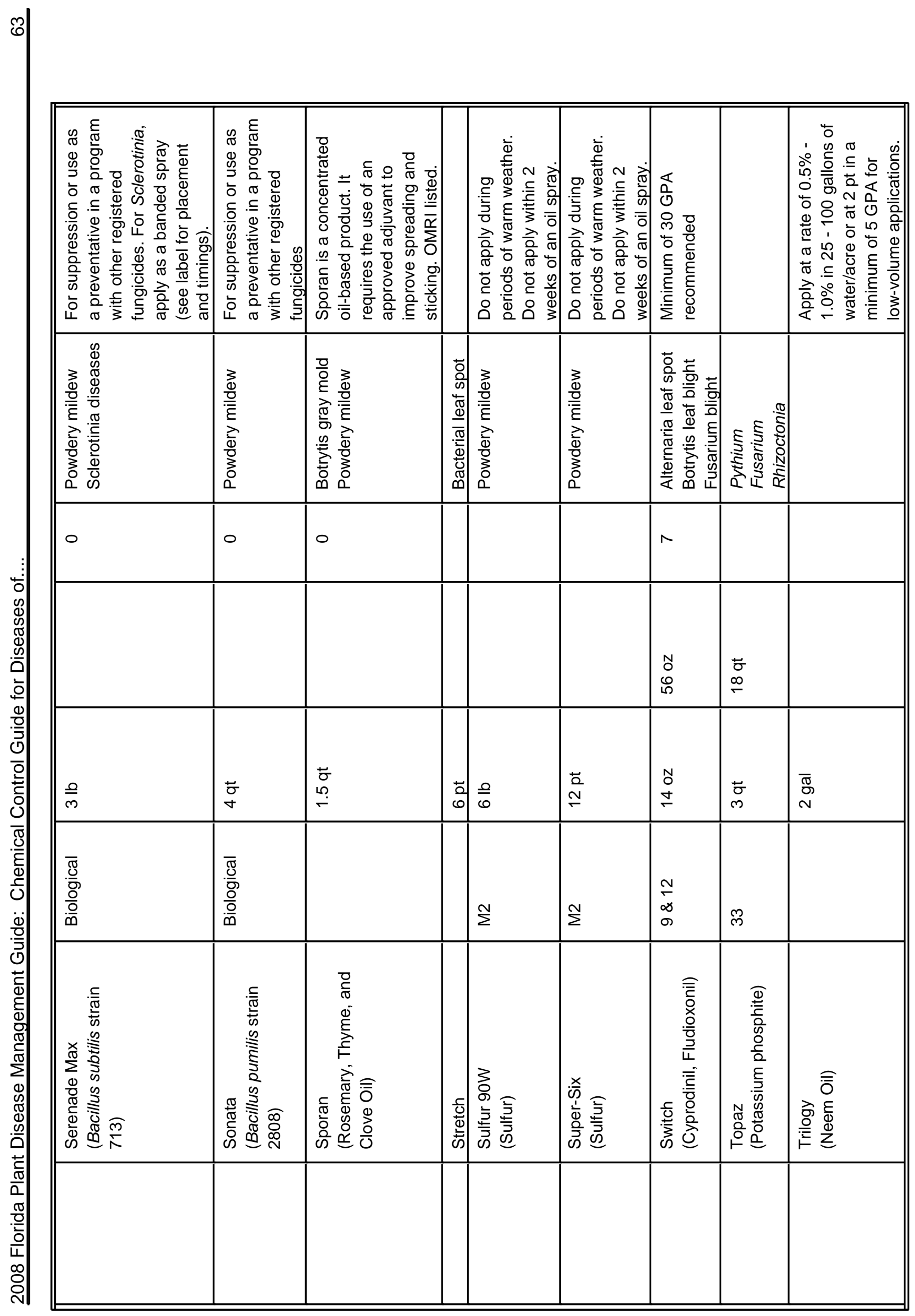




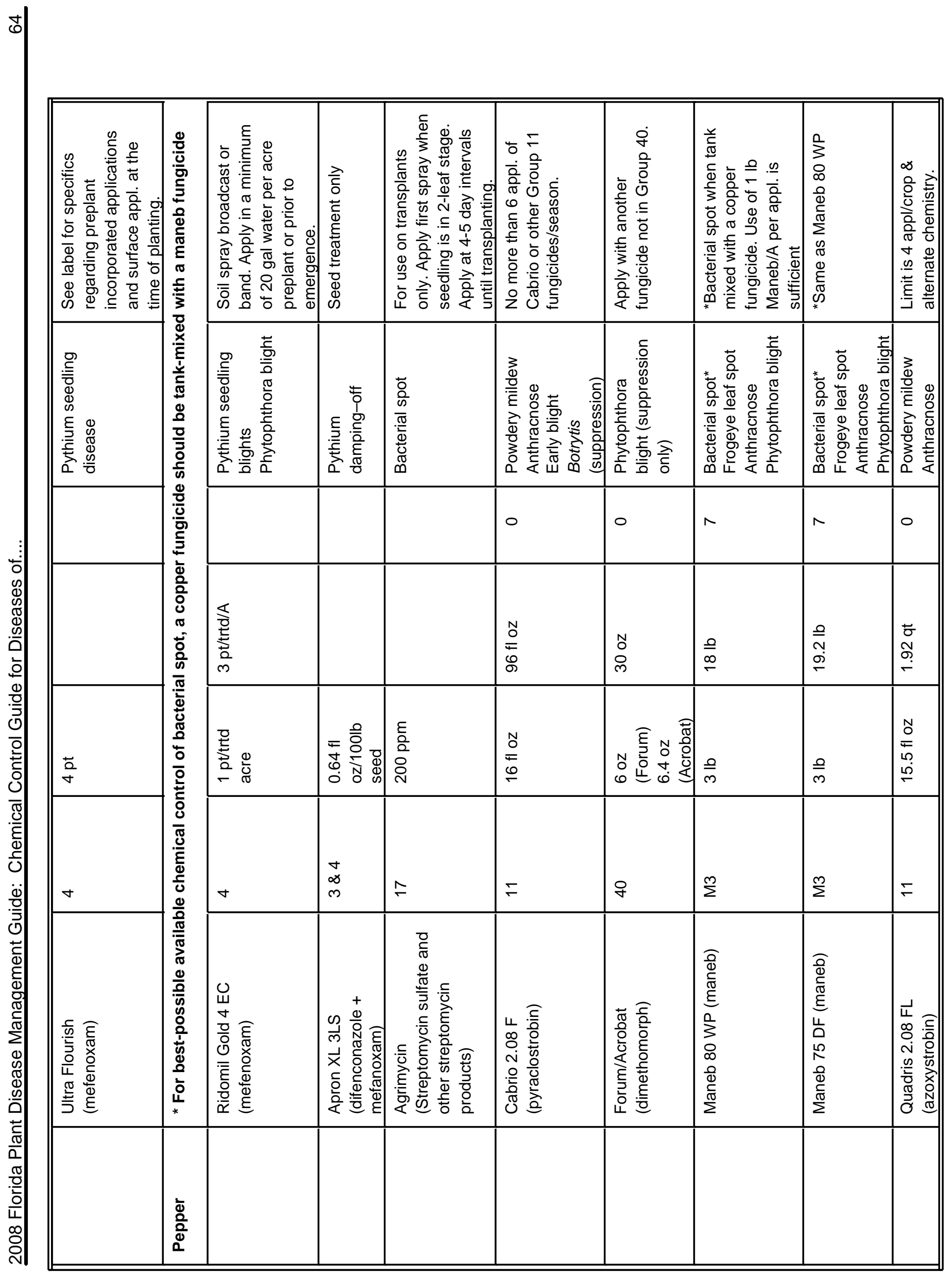




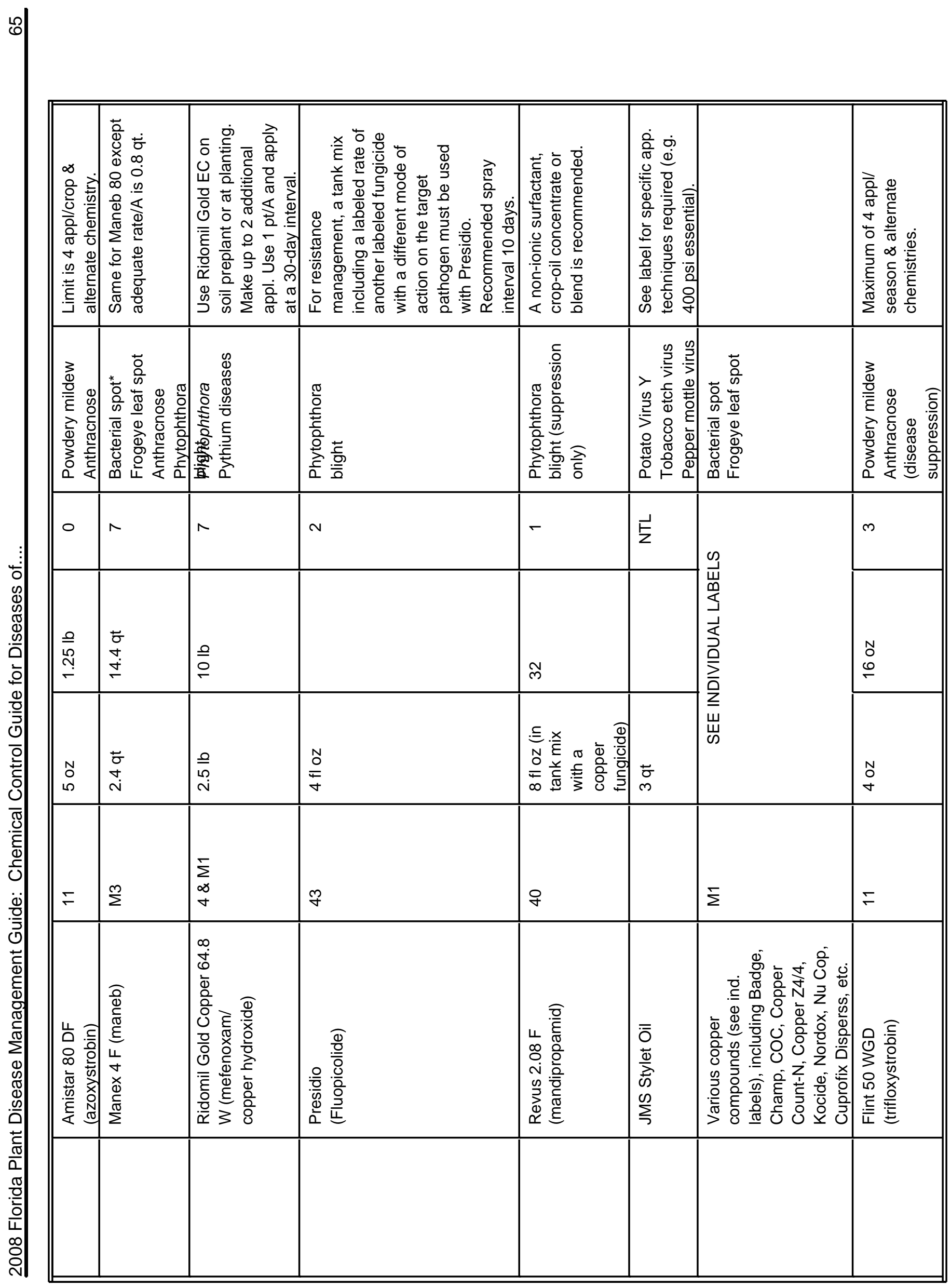




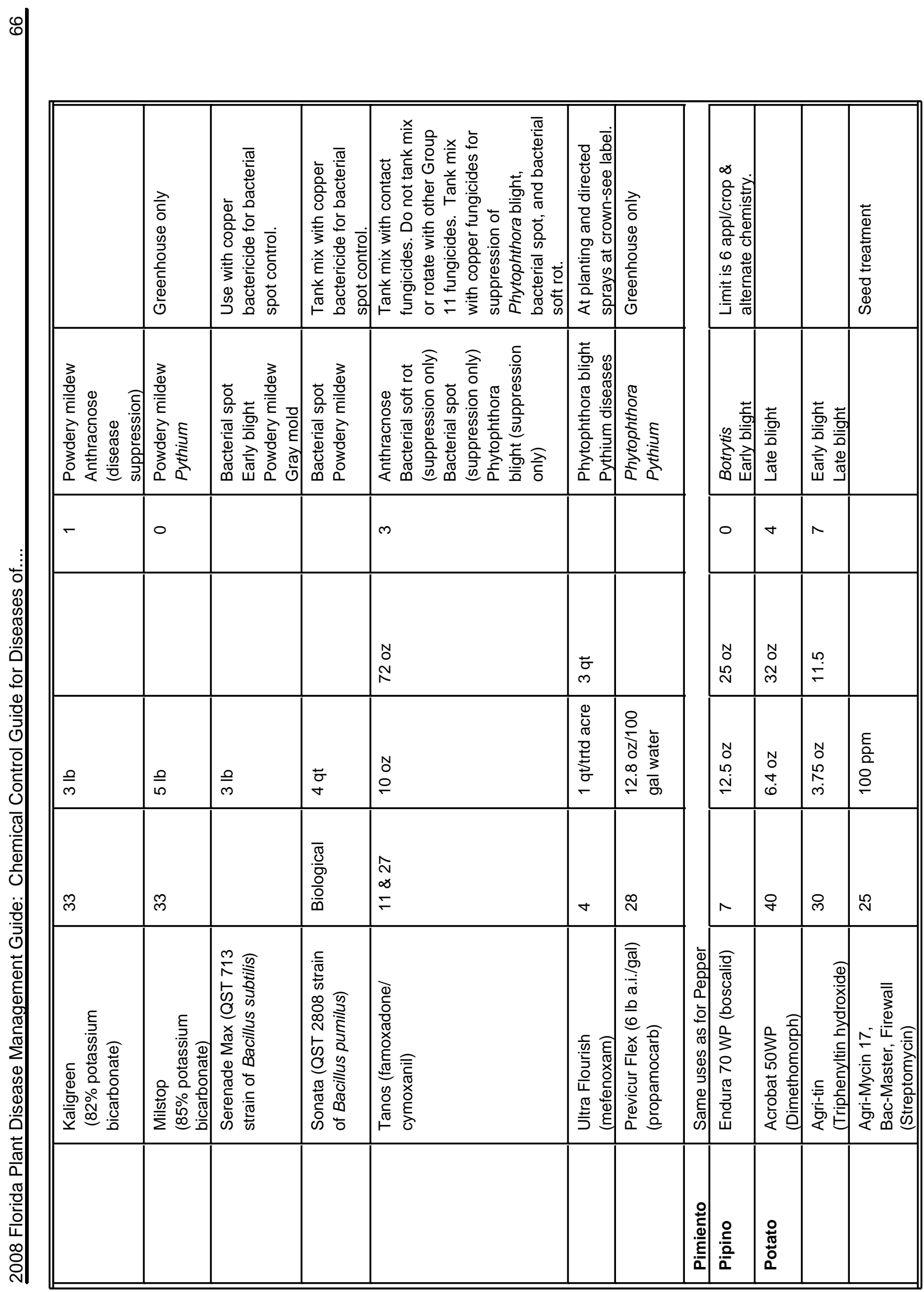




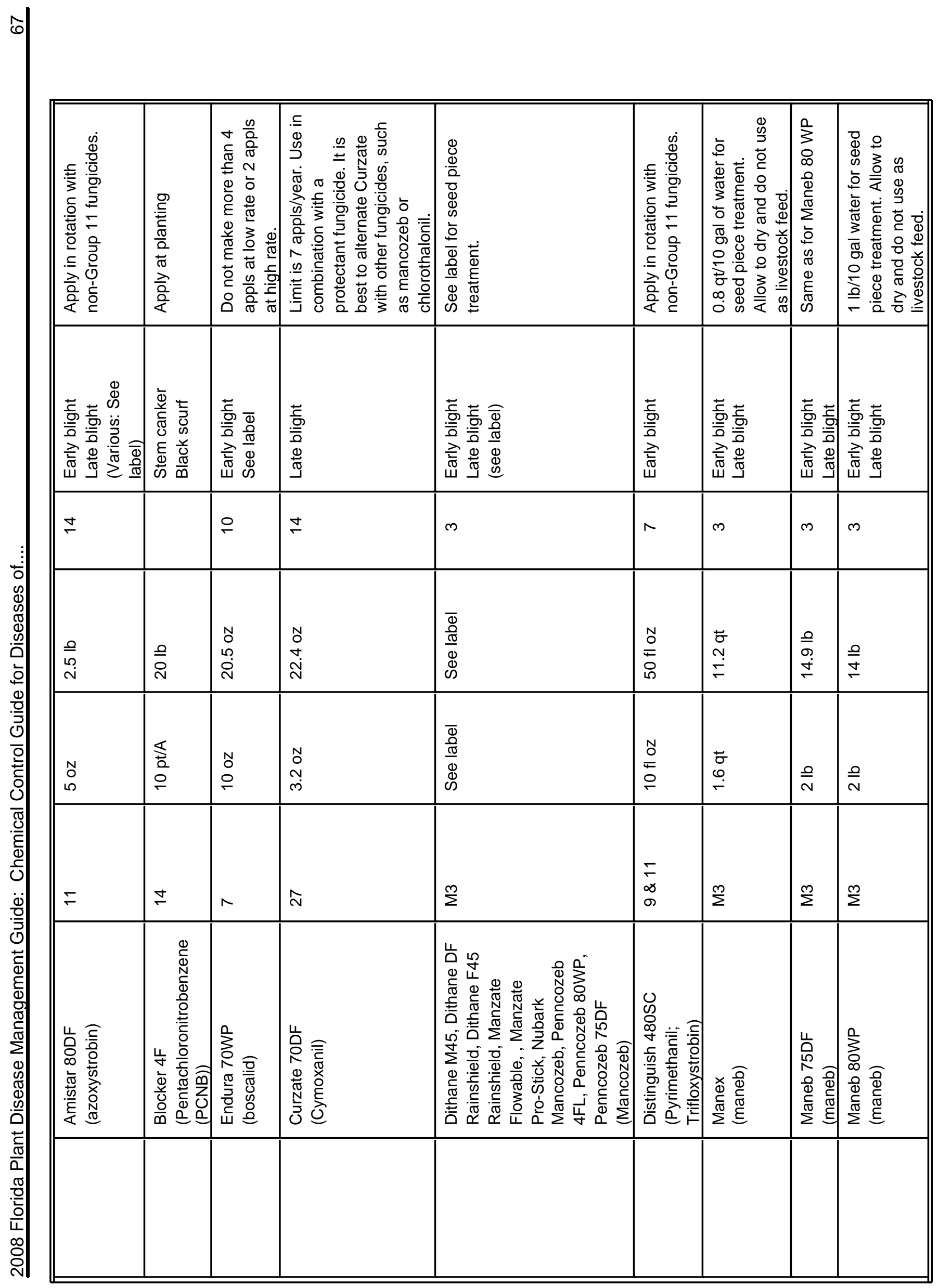




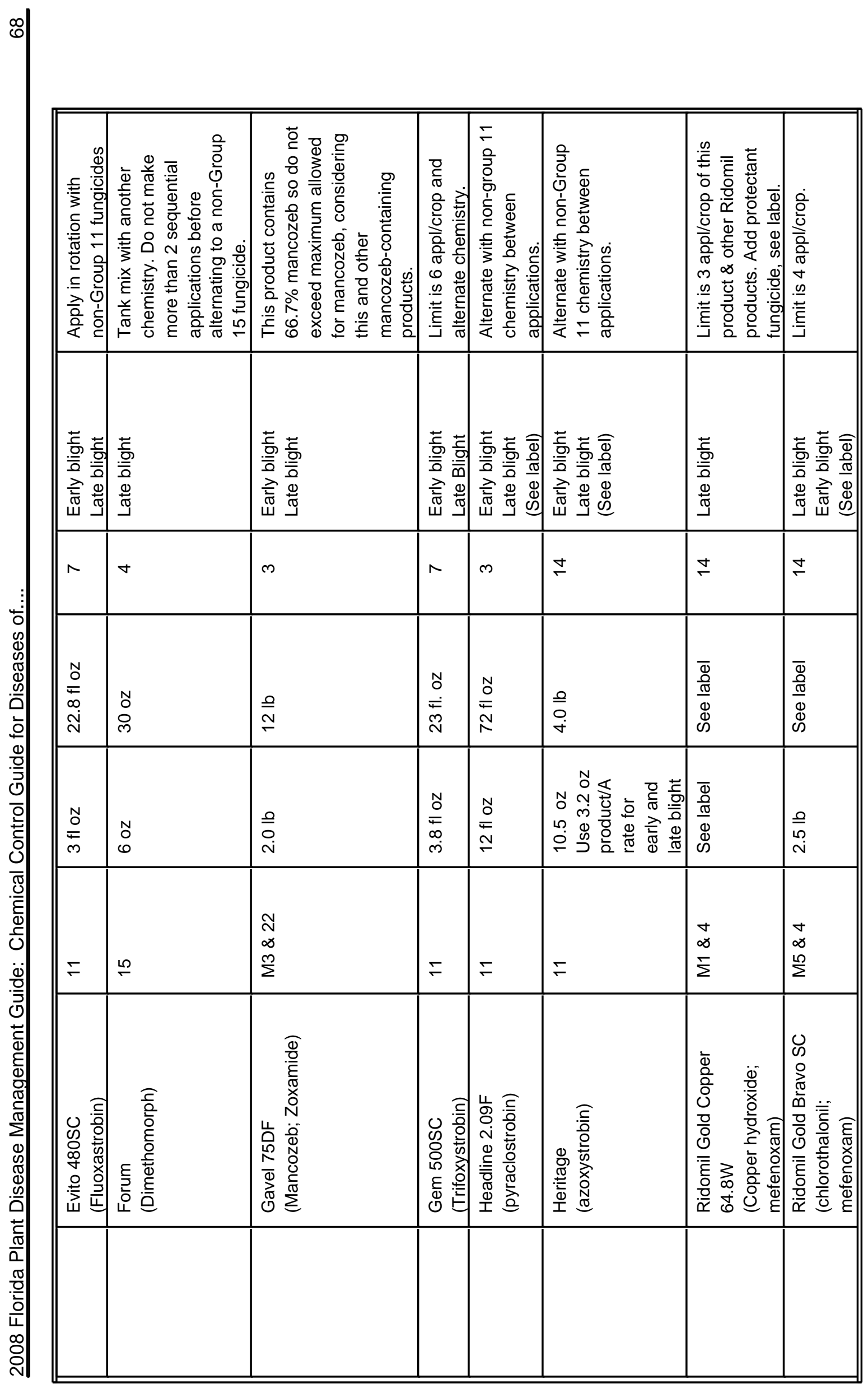




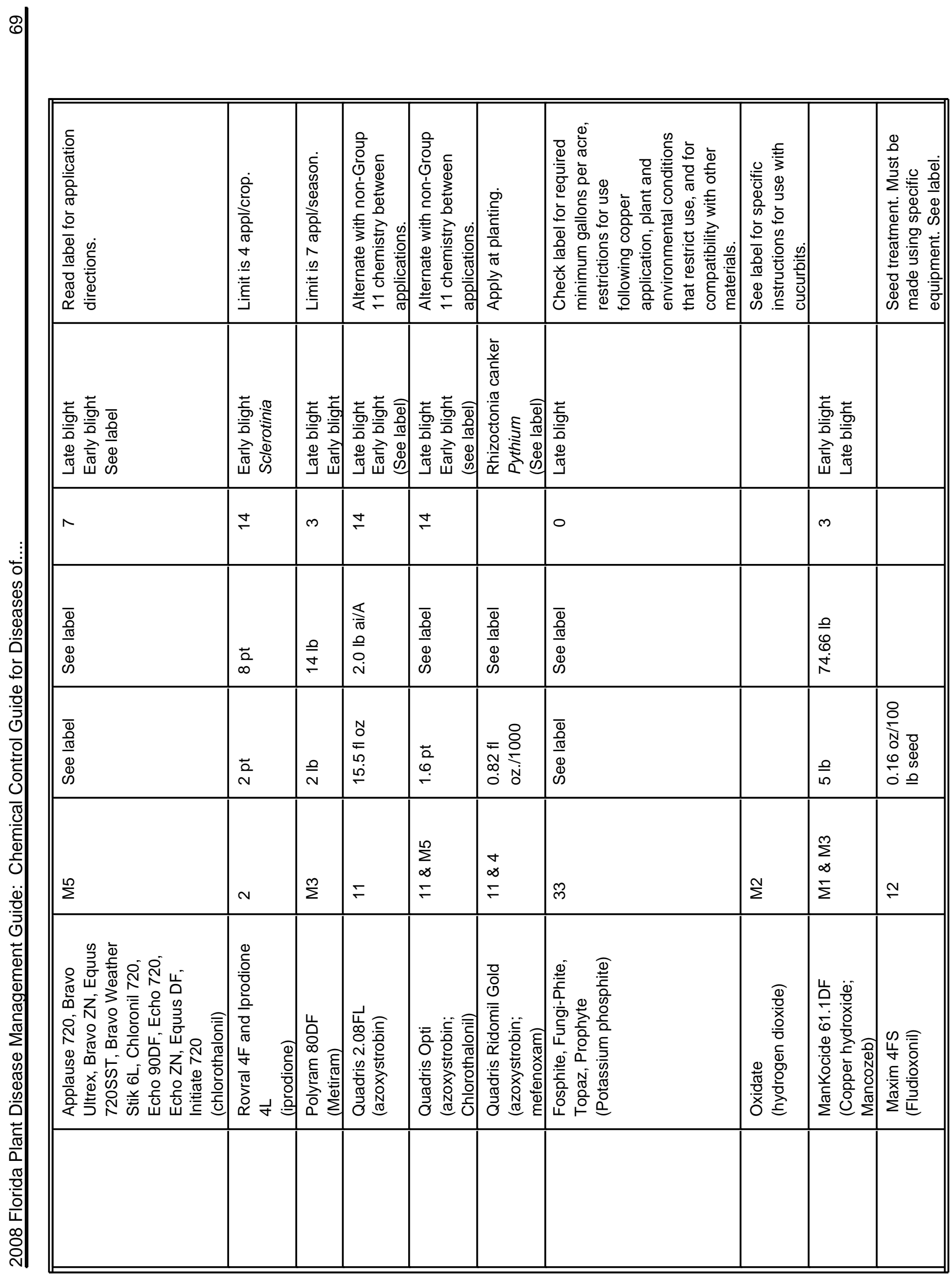




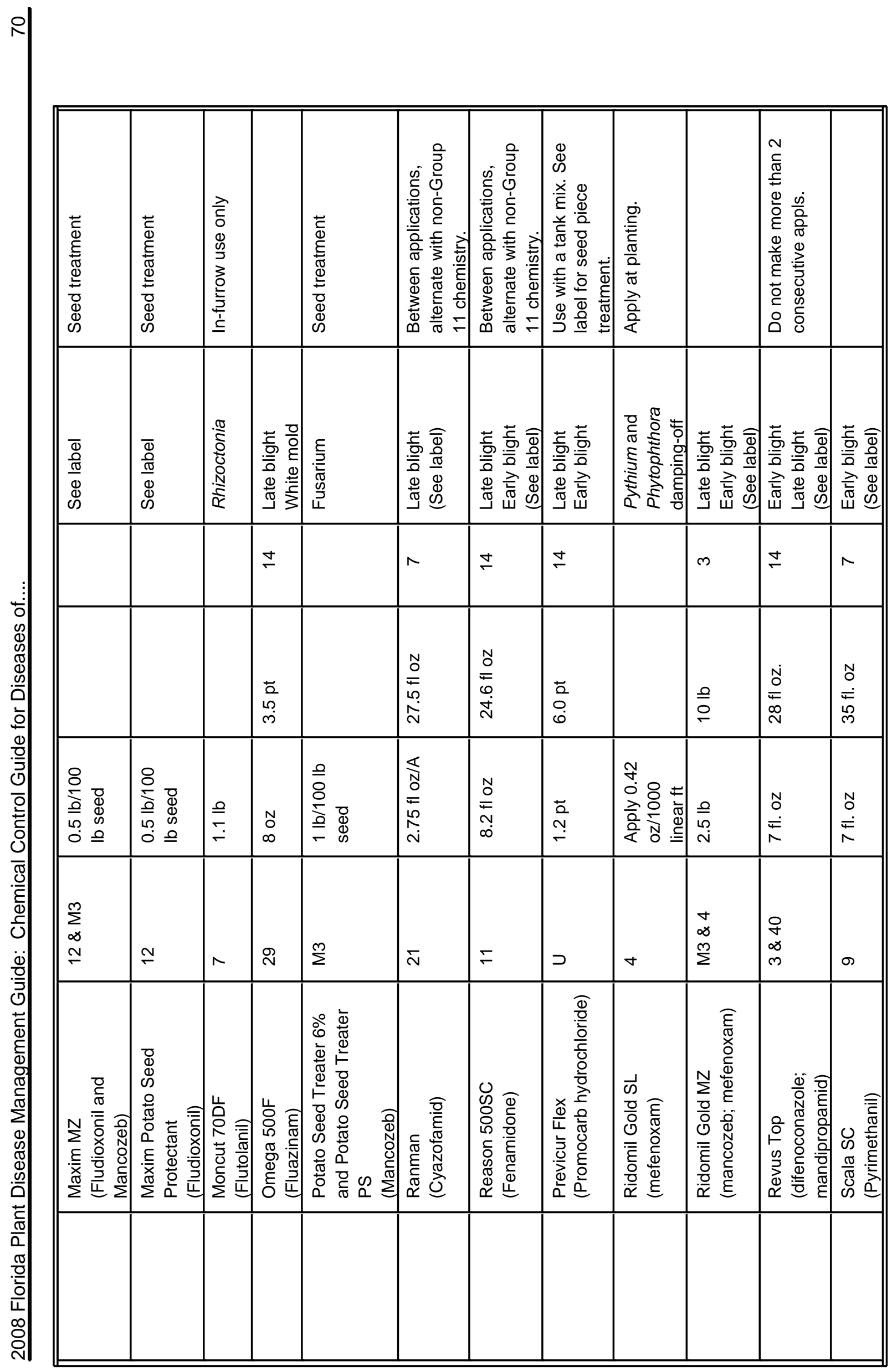




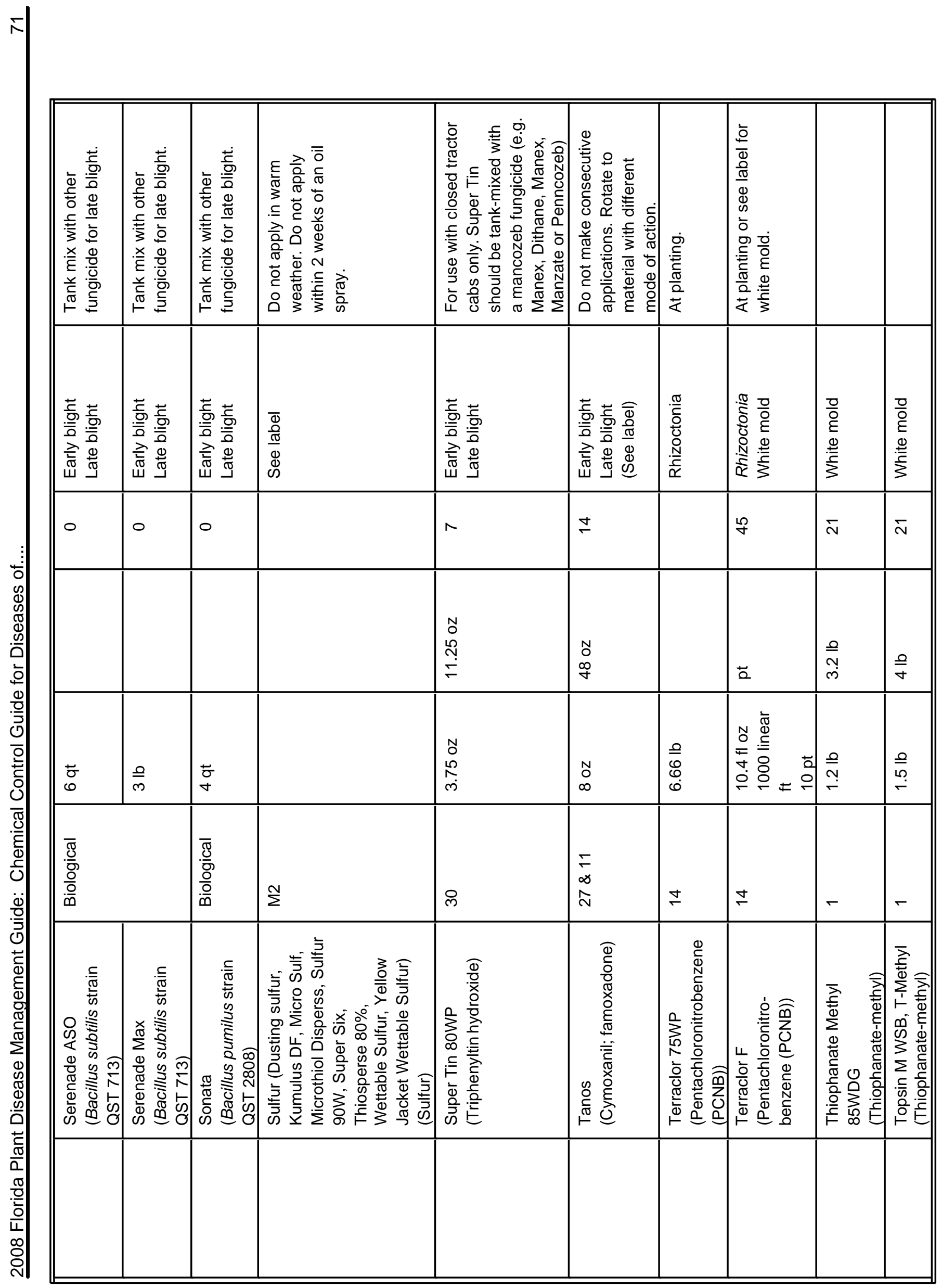




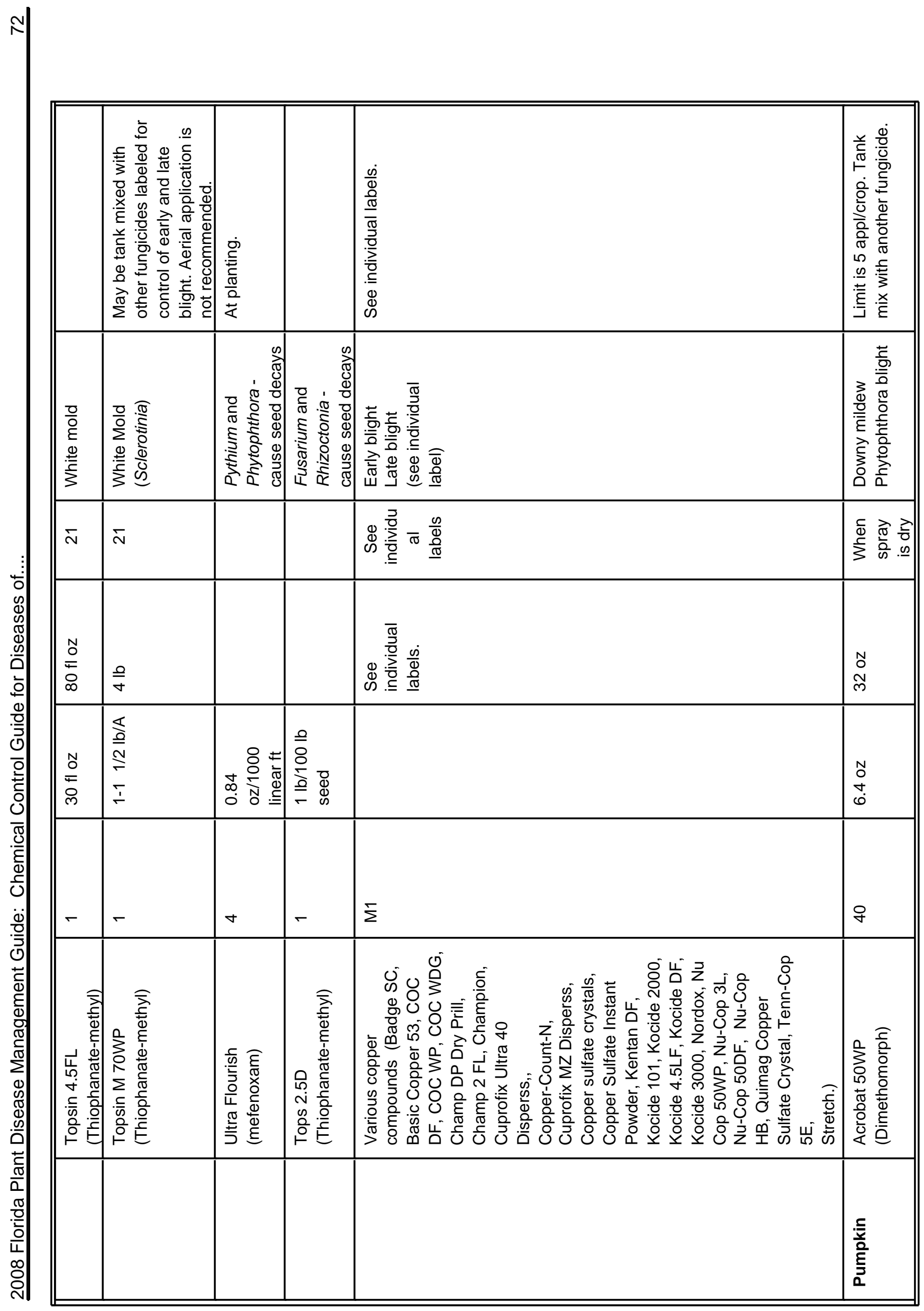




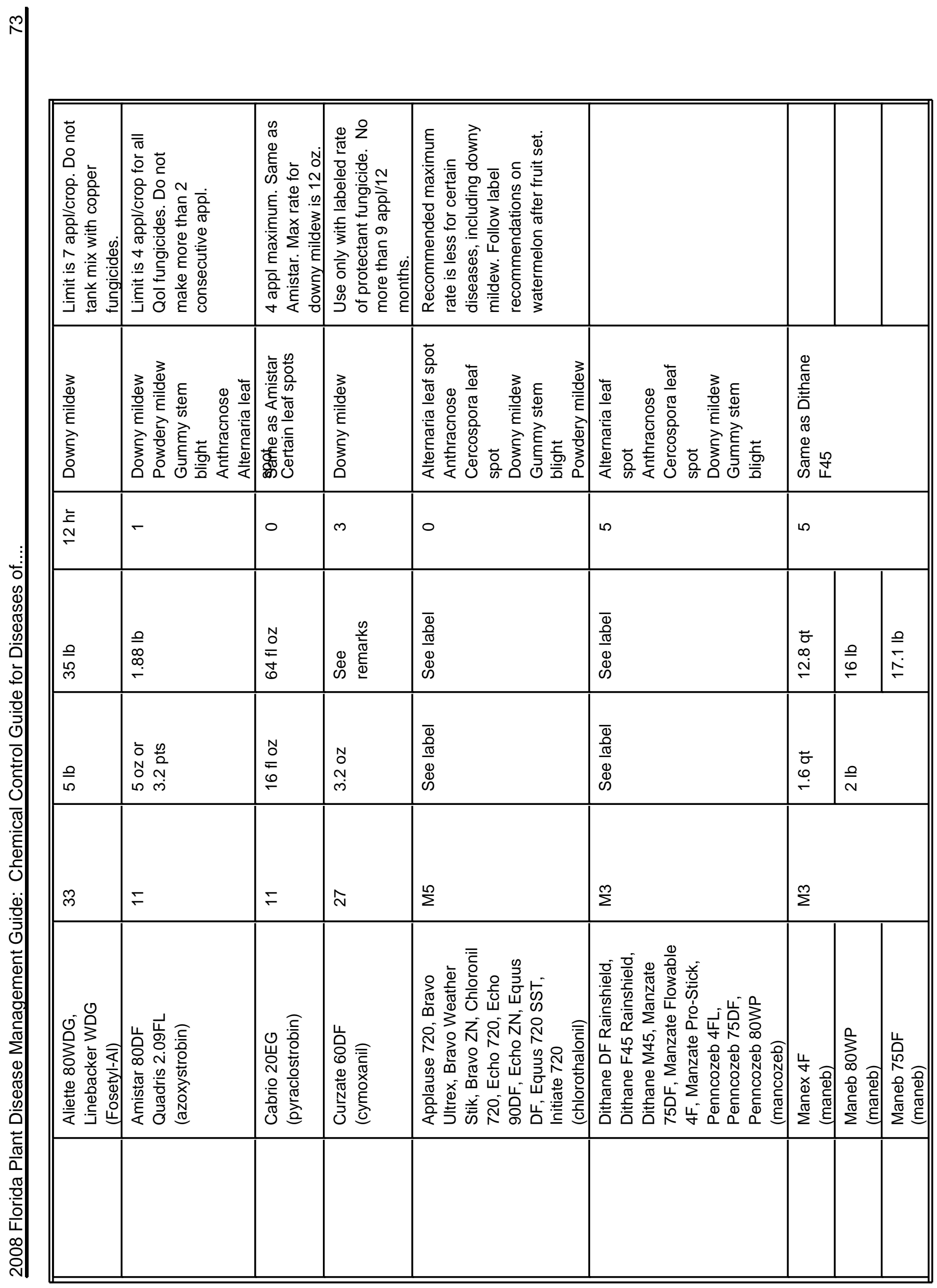




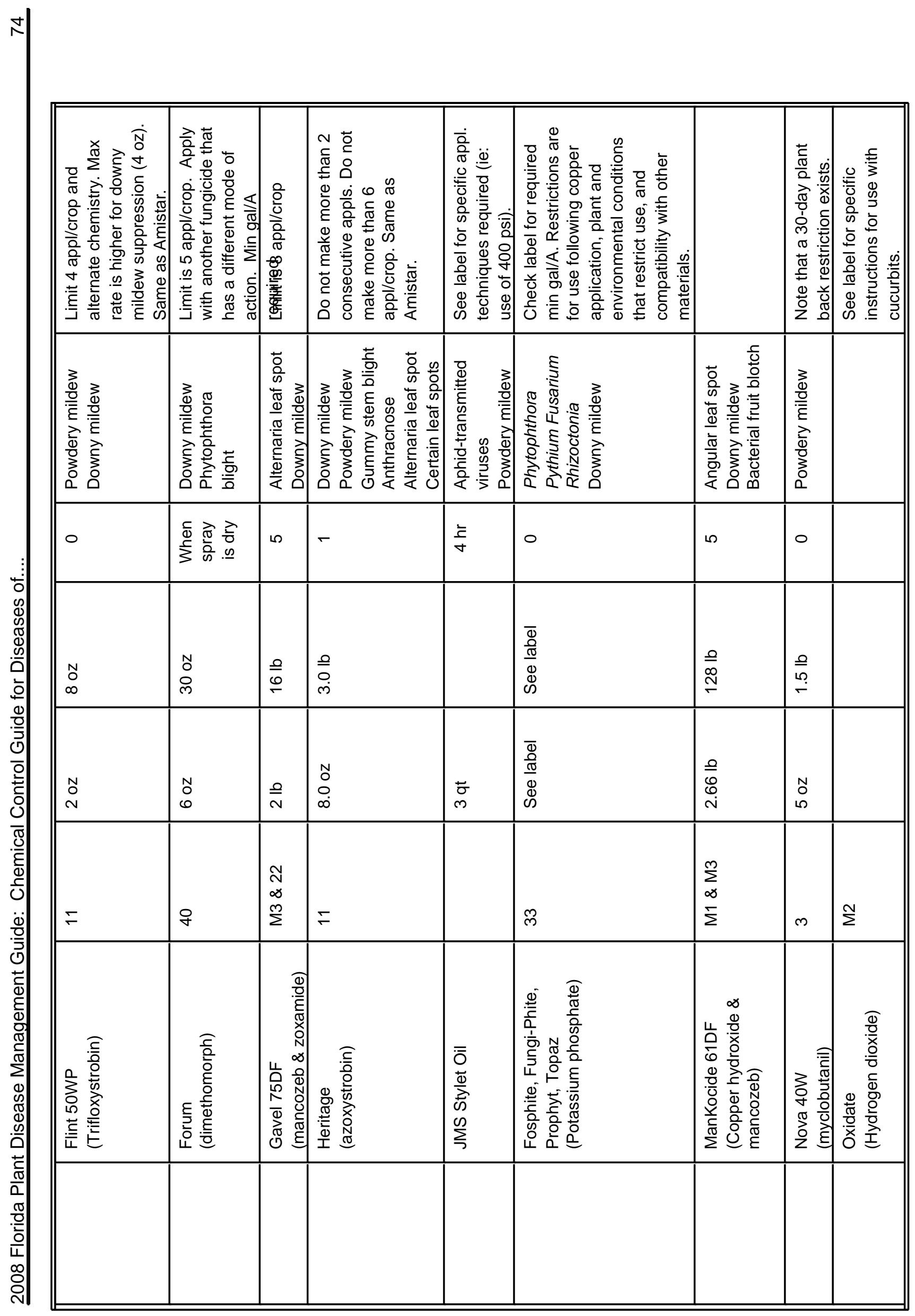




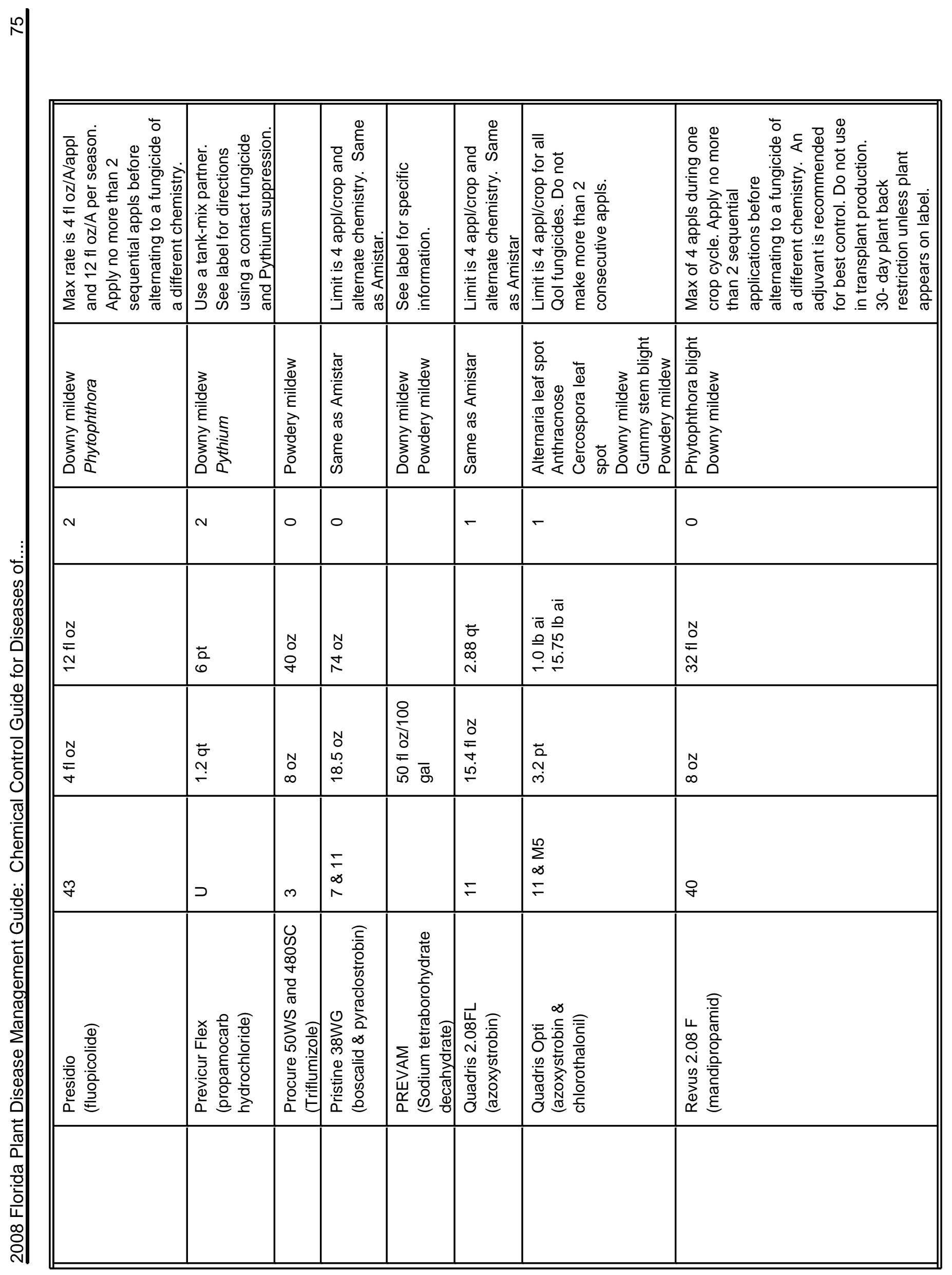




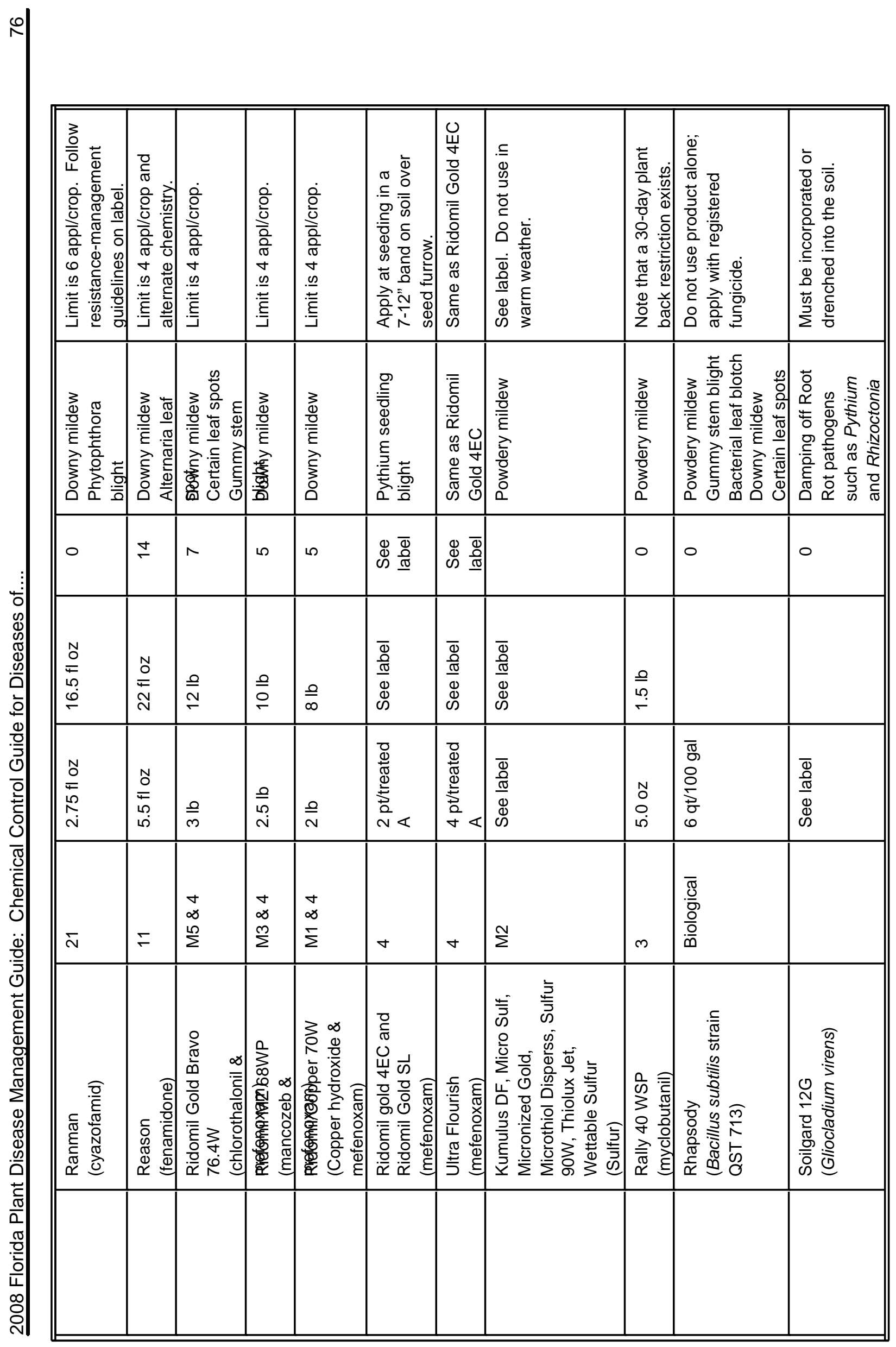




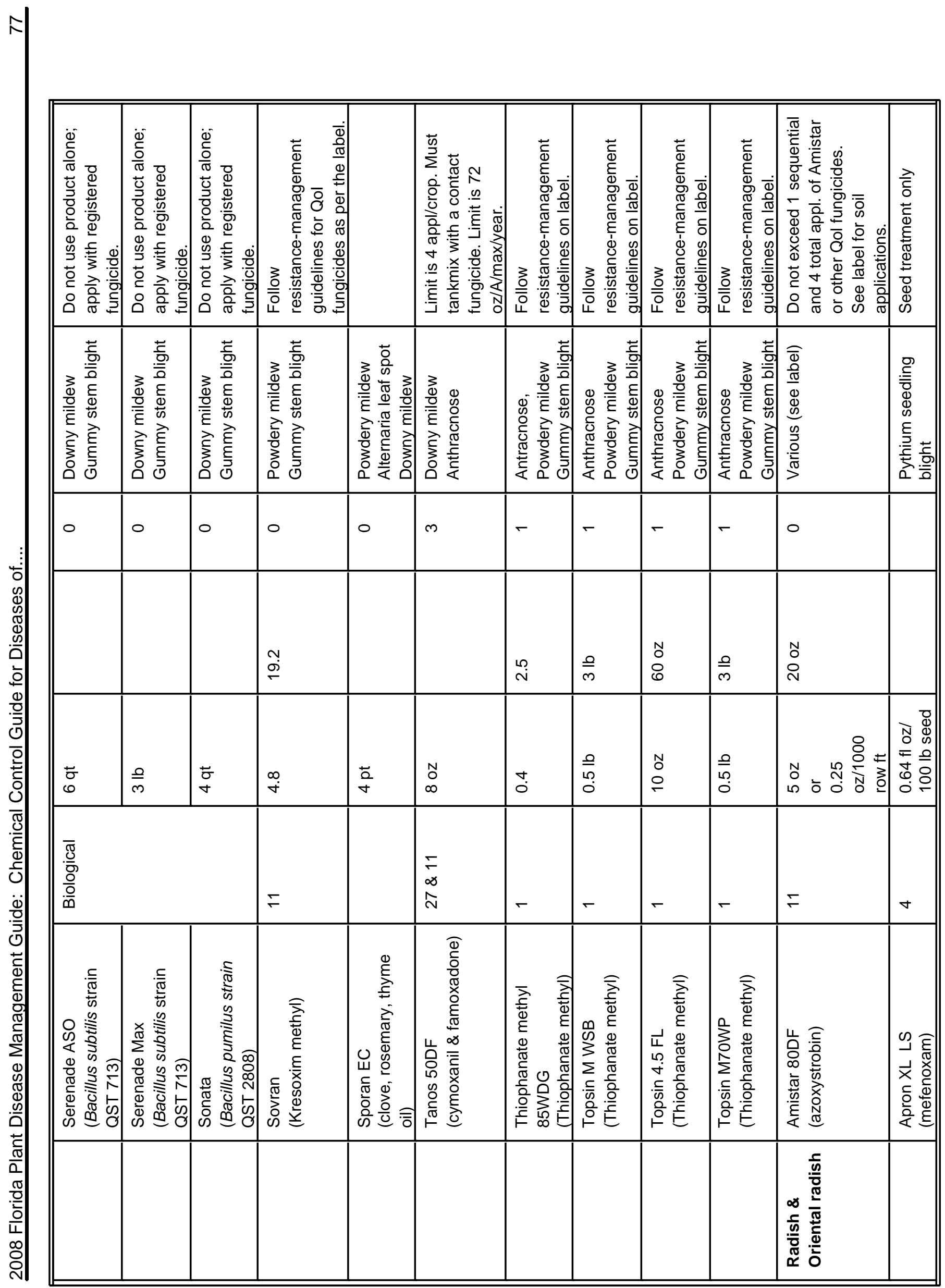




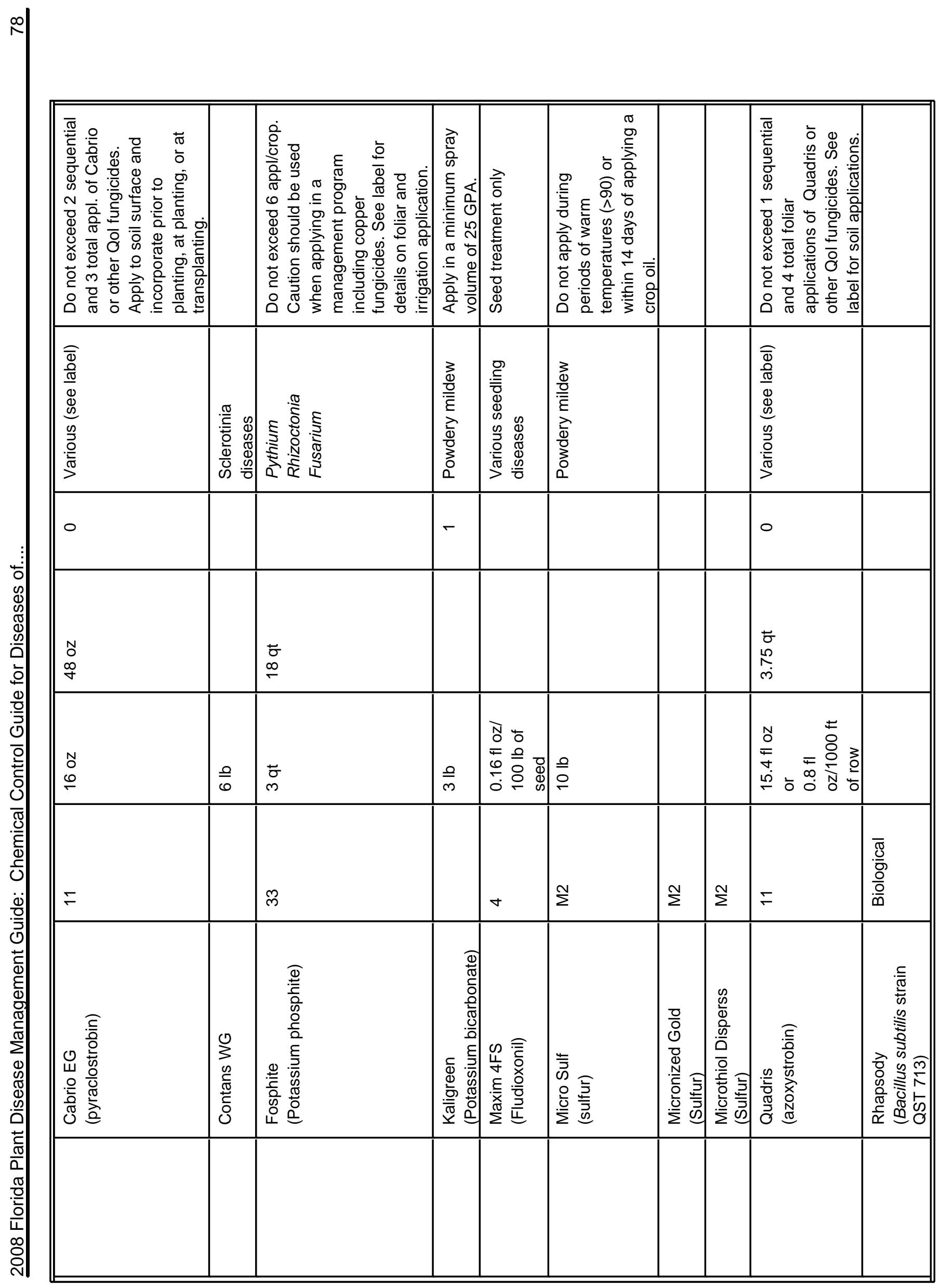




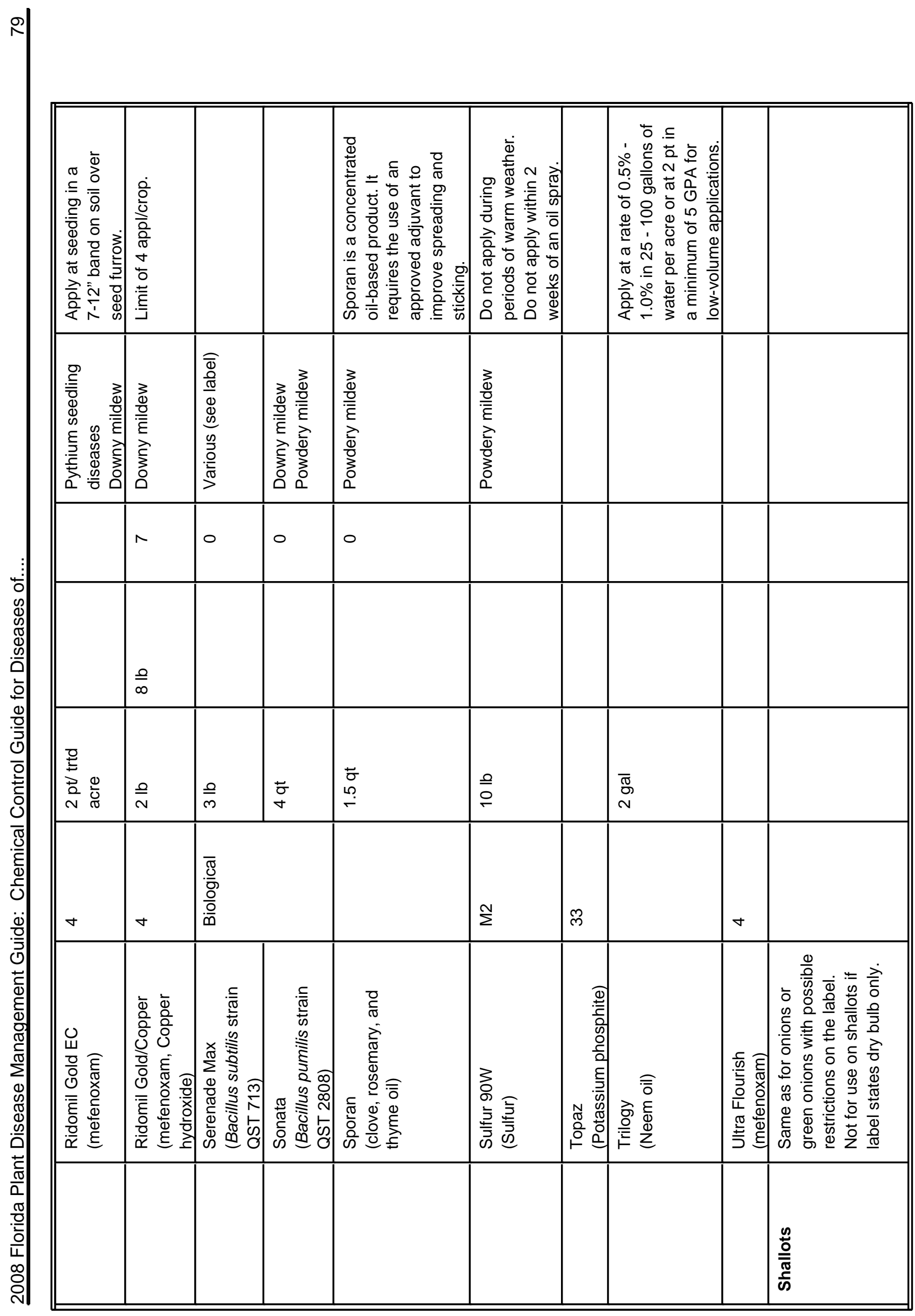




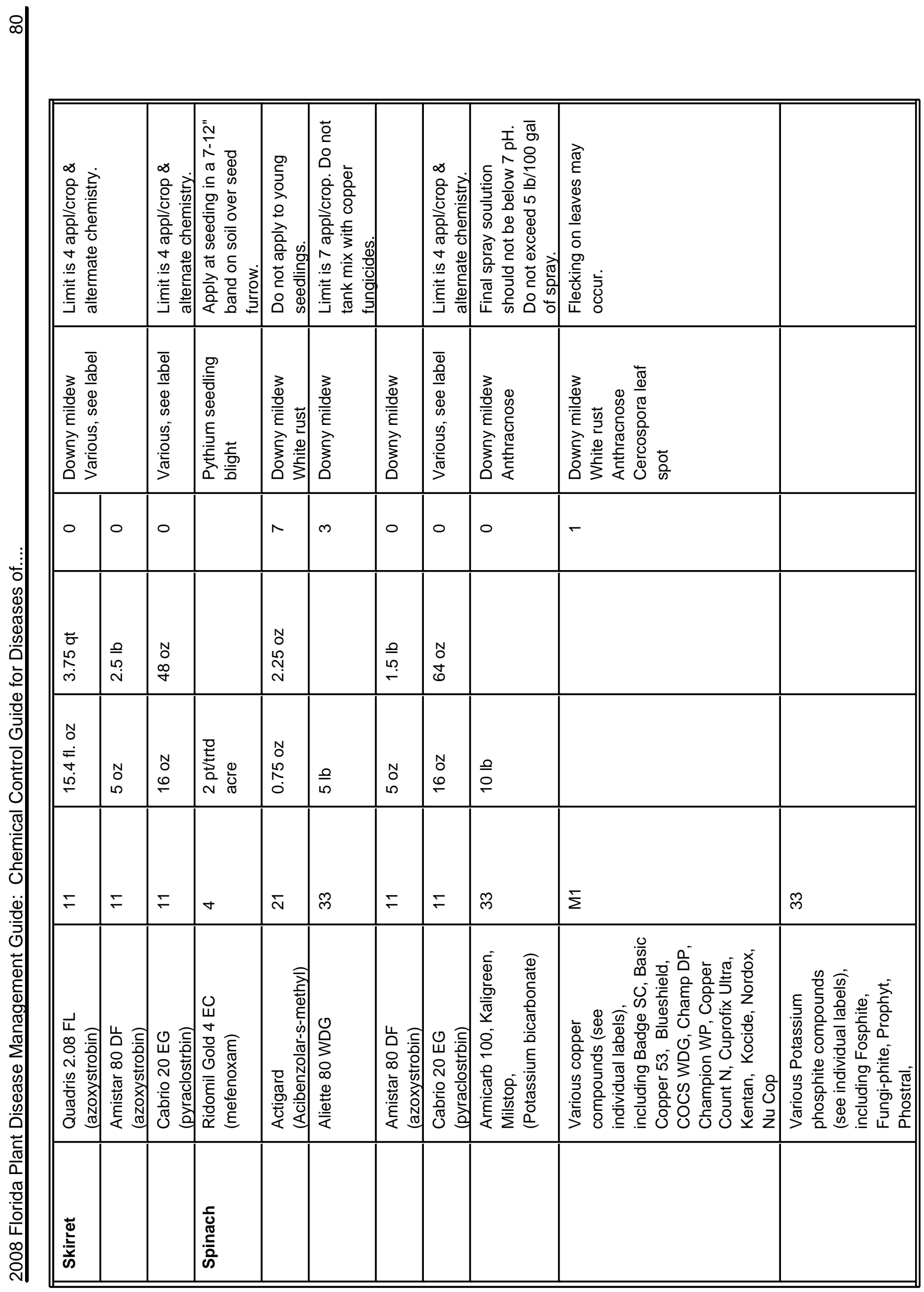




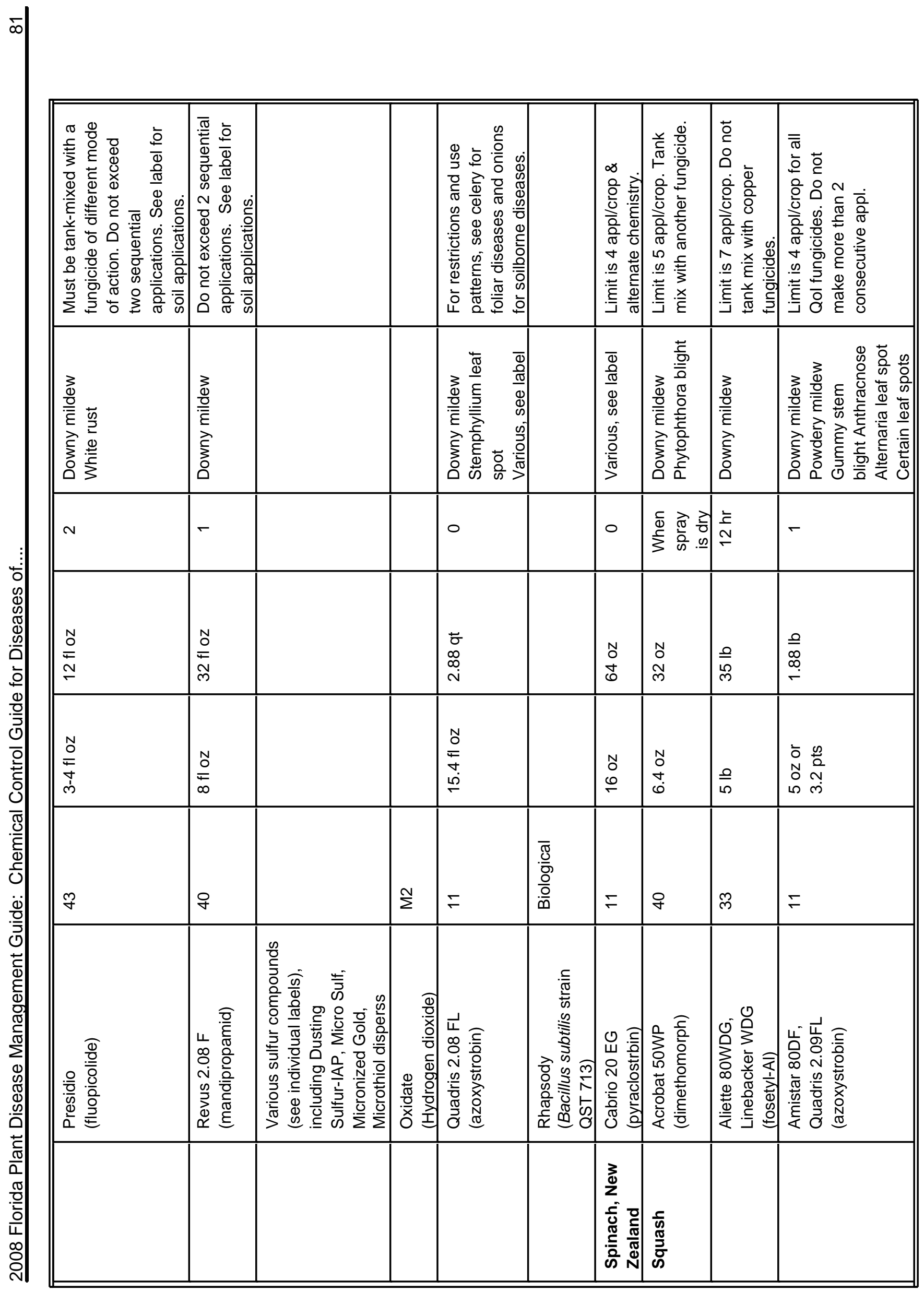




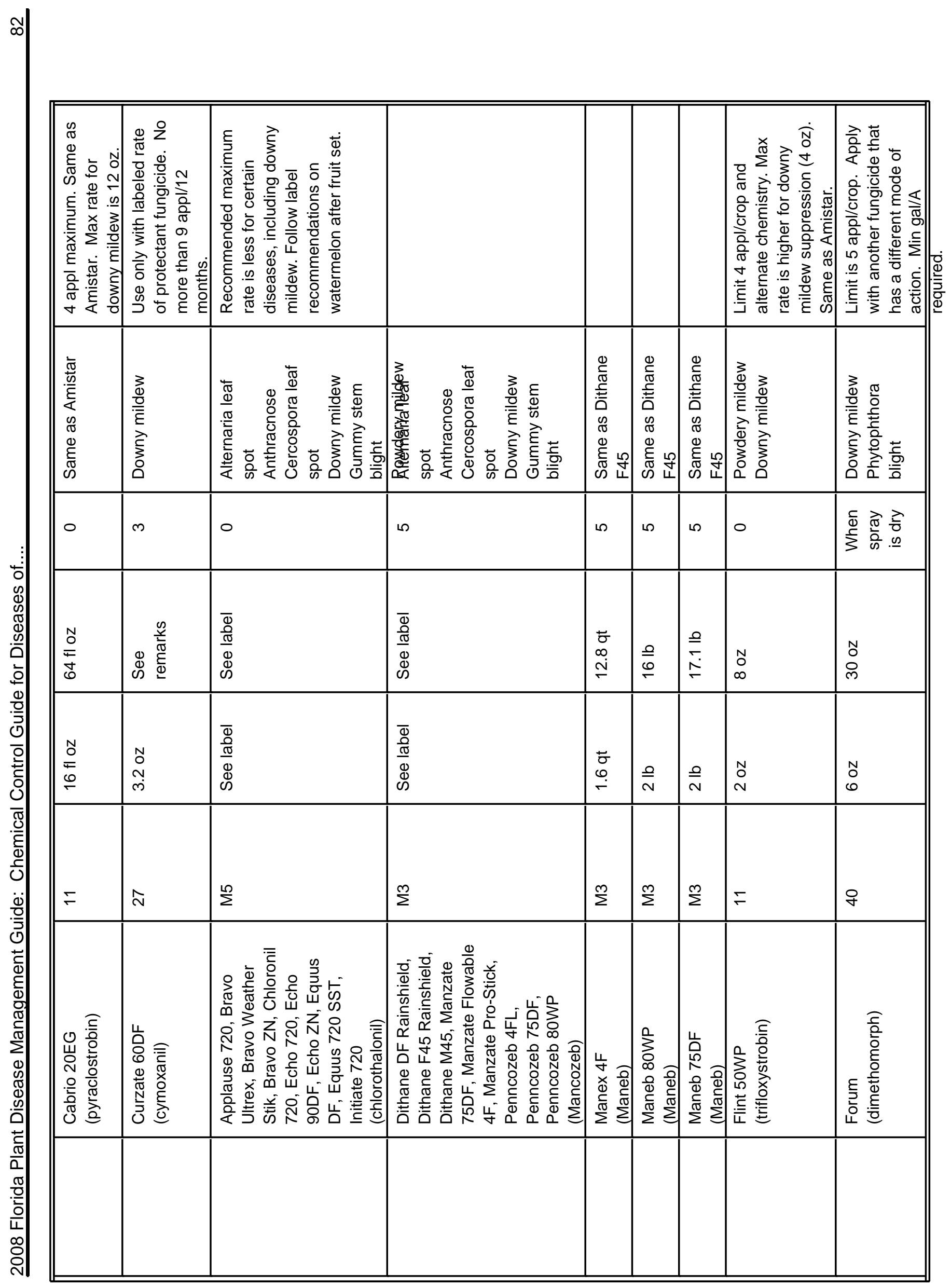




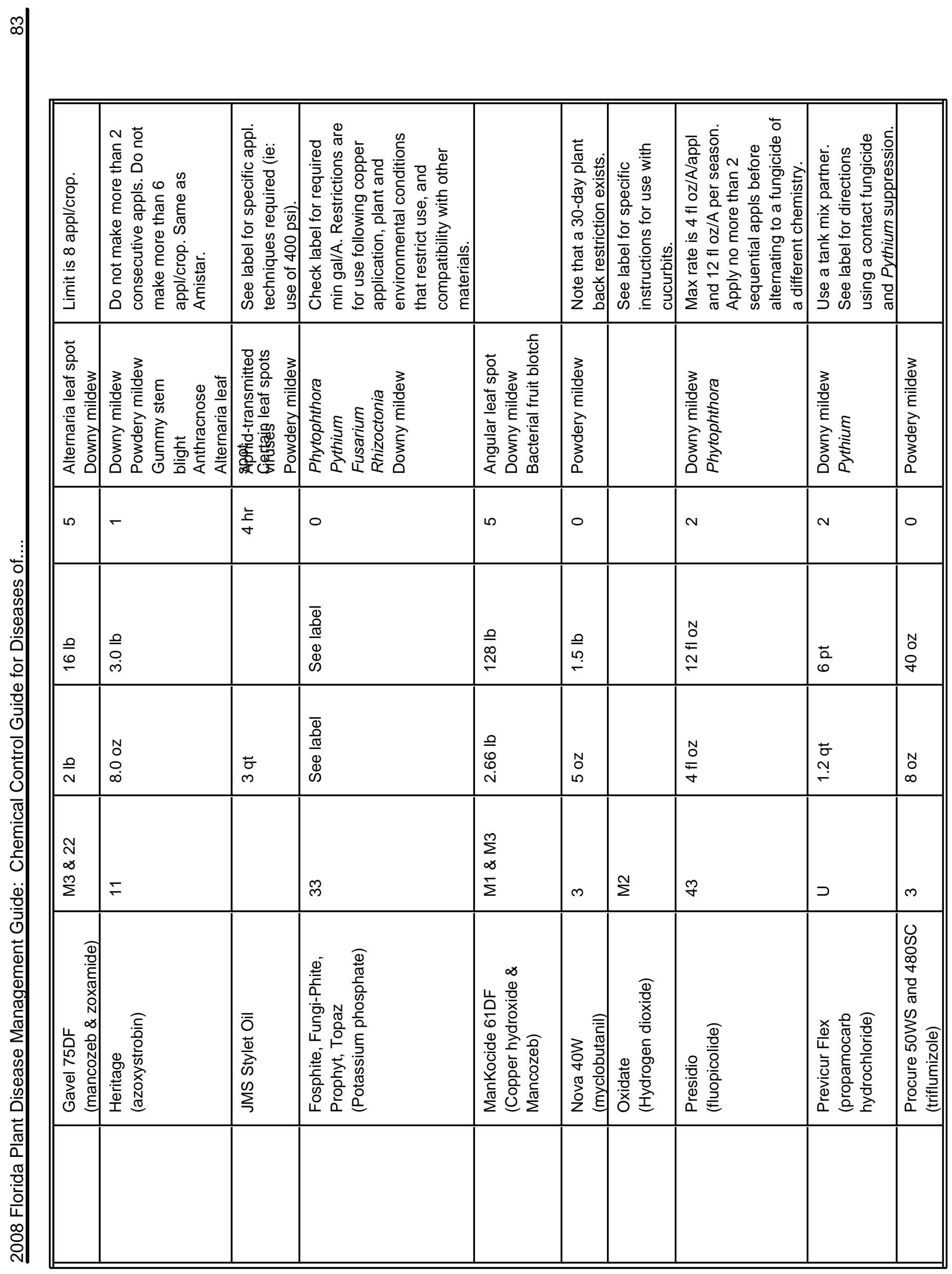




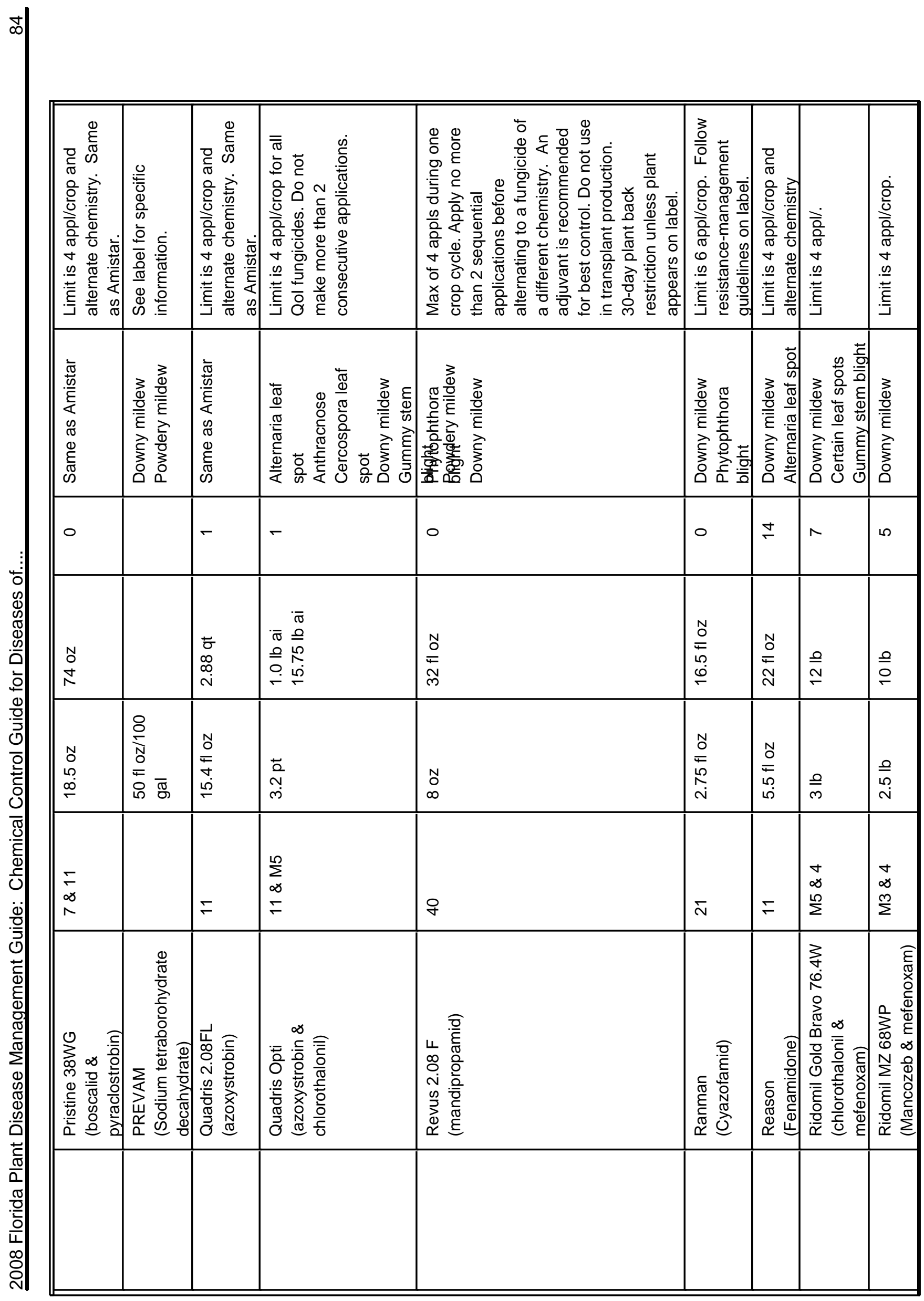




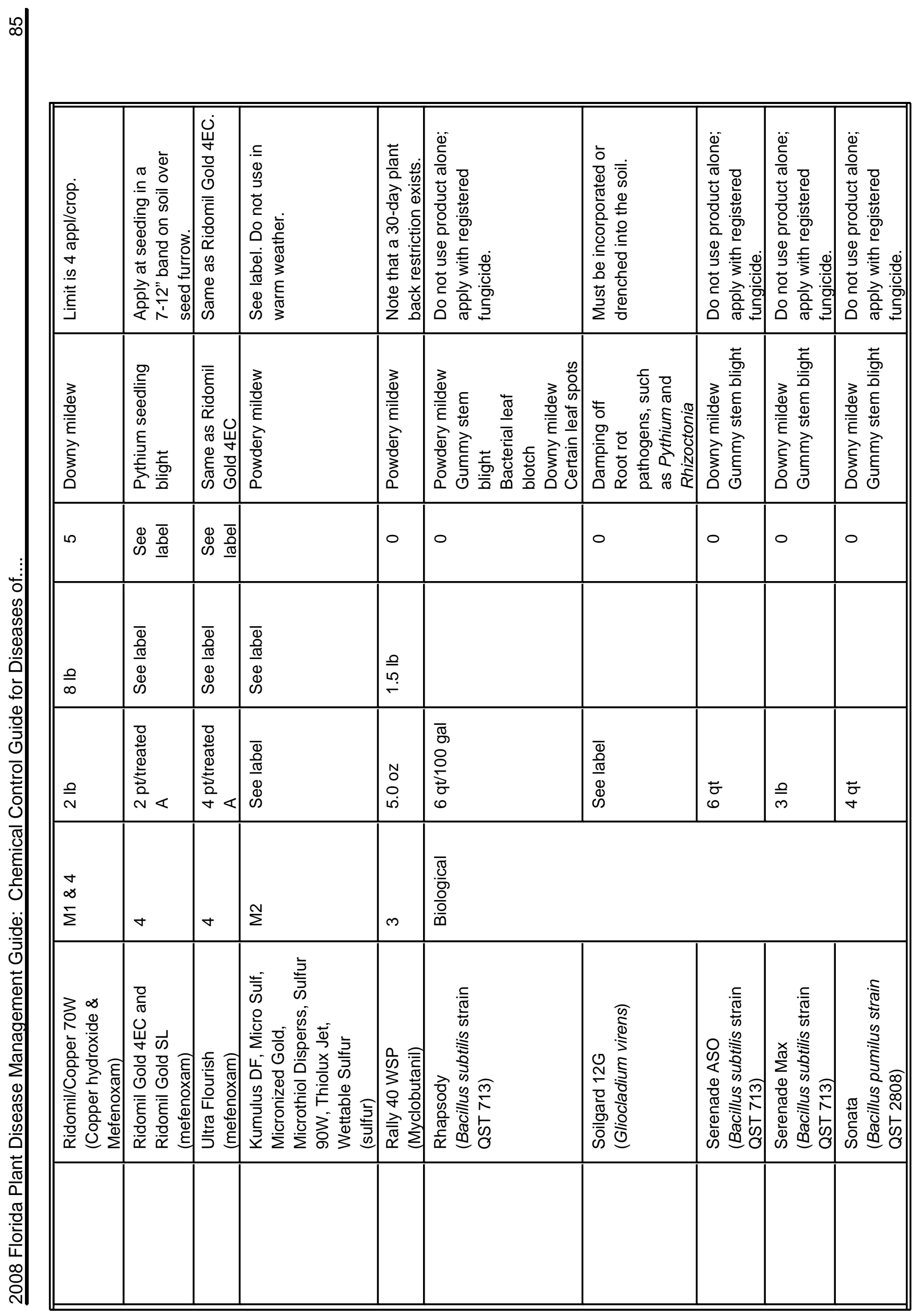




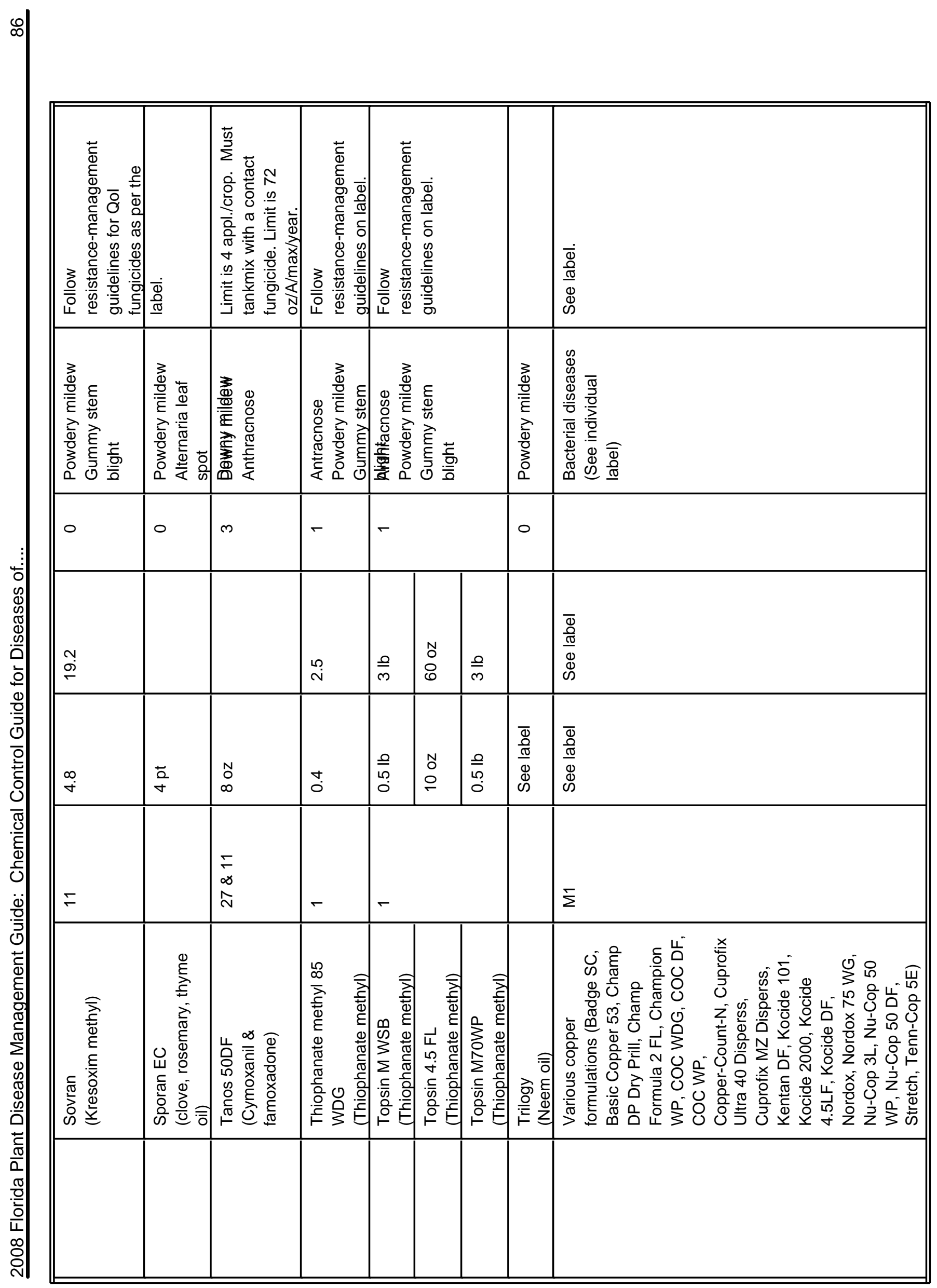




\begin{tabular}{|c|c|c|c|c|c|c|c|c|c|c|}
\hline 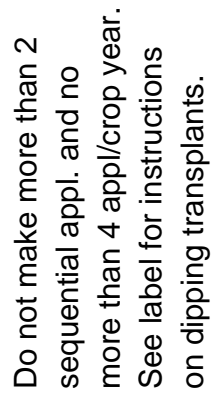 & 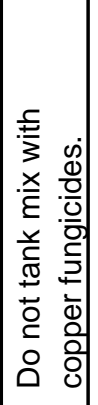 & 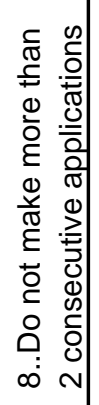 & 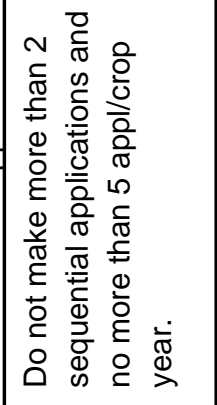 & 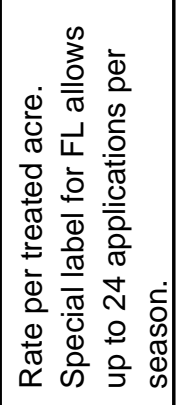 & 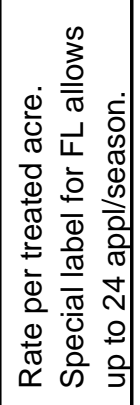 & 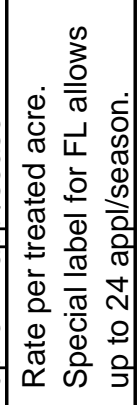 & 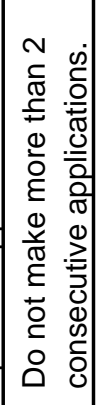 & 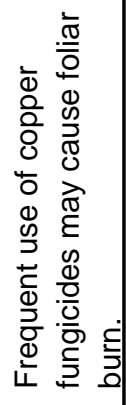 & 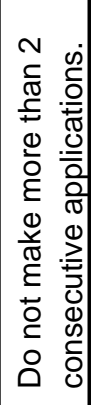 & 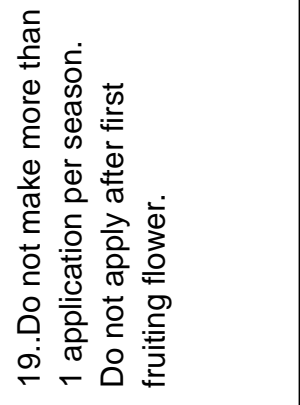 \\
\hline 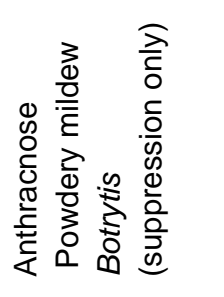 & 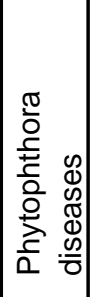 & 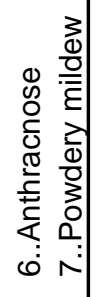 & 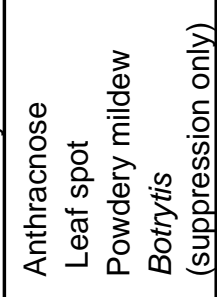 & 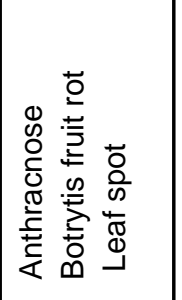 & 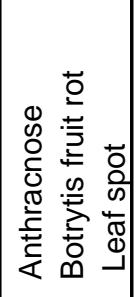 & 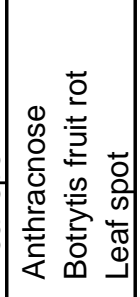 & 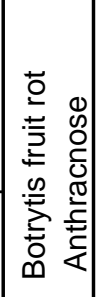 & 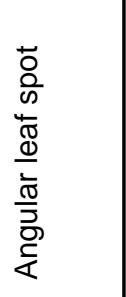 & 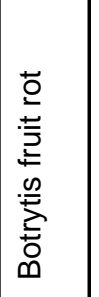 & 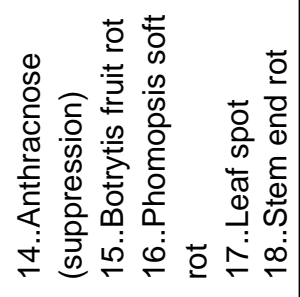 \\
\hline 0 & $\begin{array}{l}\grave{\Sigma} \\
\stackrel{\Sigma}{\simeq}\end{array}$ & $\begin{array}{l}0 \\
\text { i: }\end{array}$ & 0 & - & - & - & 0 & $\stackrel{\sim}{\leftarrow}$ & 0 & 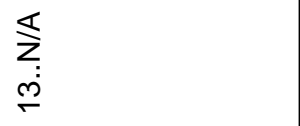 \\
\hline 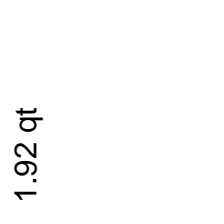 & 吕 & $\begin{array}{l}\stackrel{N}{O} \\
\underset{\leftarrow}{0} \\
\stackrel{+}{+}\end{array}$ & 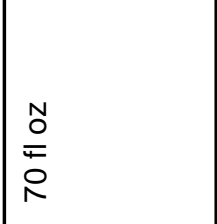 & 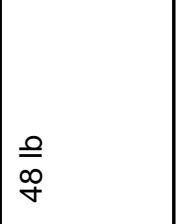 & 응 & $\begin{array}{l}\vec{\sigma} \\
\stackrel{\sim}{N}\end{array}$ & $\stackrel{\rho}{\bar{N}}$ & 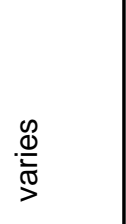 & $\frac{0}{6}$ & 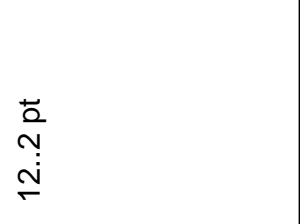 \\
\hline 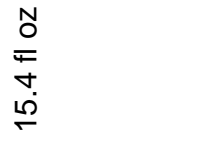 & $\frac{0}{10}$ & $\begin{array}{l}N \\
\stackrel{N}{+} \\
\dot{\vdots} \\
\dot{0}\end{array}$ & $\begin{array}{l}\stackrel{N}{E} \\
\stackrel{+}{ \pm}\end{array}$ & $\stackrel{0}{6}$ & $\begin{array}{l}\stackrel{\rho}{\frac{\rho}{\rho}} \\
\stackrel{\rho}{m}\end{array}$ & $\begin{array}{l}\vec{\sigma} \\
\text { m }\end{array}$ & $\begin{array}{l}\stackrel{0}{\nu} \\
\stackrel{N}{n} \\
\stackrel{\circ}{\circ}\end{array}$ & $\frac{\mathscr{\mathscr { U }}}{\stackrel{\frac{\pi}{\pi}}{>}}$ & $\begin{array}{l}\stackrel{0}{\circ} \\
\stackrel{\sim}{-}\end{array}$ & 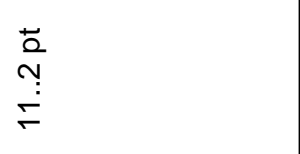 \\
\hline$\mp$ & लm & $\stackrel{m}{\sim}$ & $\mp$ & $\stackrel{m}{\Sigma}$ & $\sum^{m}$ & $\sum^{M}$ & $\begin{array}{l}N \\
\infty \\
\sum \\
\Sigma\end{array}$ & $\begin{array}{l}\sum^{0} \\
\overline{0} \\
\sum\end{array}$ & $\stackrel{\sim}{\perp}$ & $\begin{array}{l}\text { O̊: } \\
\stackrel{0}{0}\end{array}$ \\
\hline 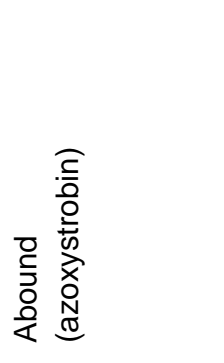 & 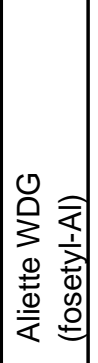 & 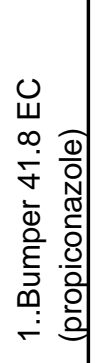 & 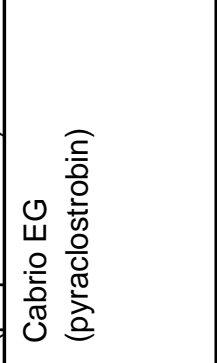 & 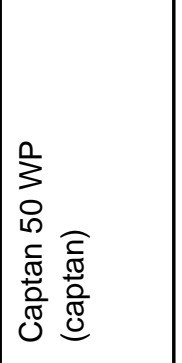 & 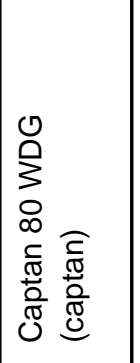 & 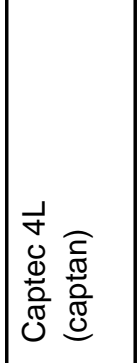 & 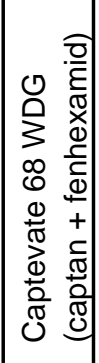 & 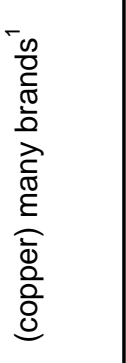 & 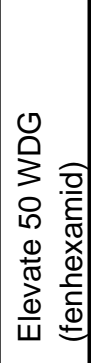 & 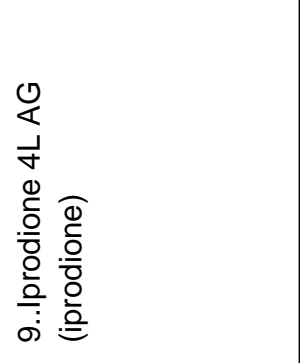 \\
\hline 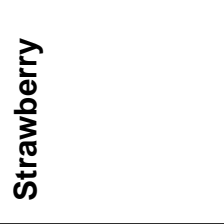 & & & & & & & & & & \\
\hline
\end{tabular}




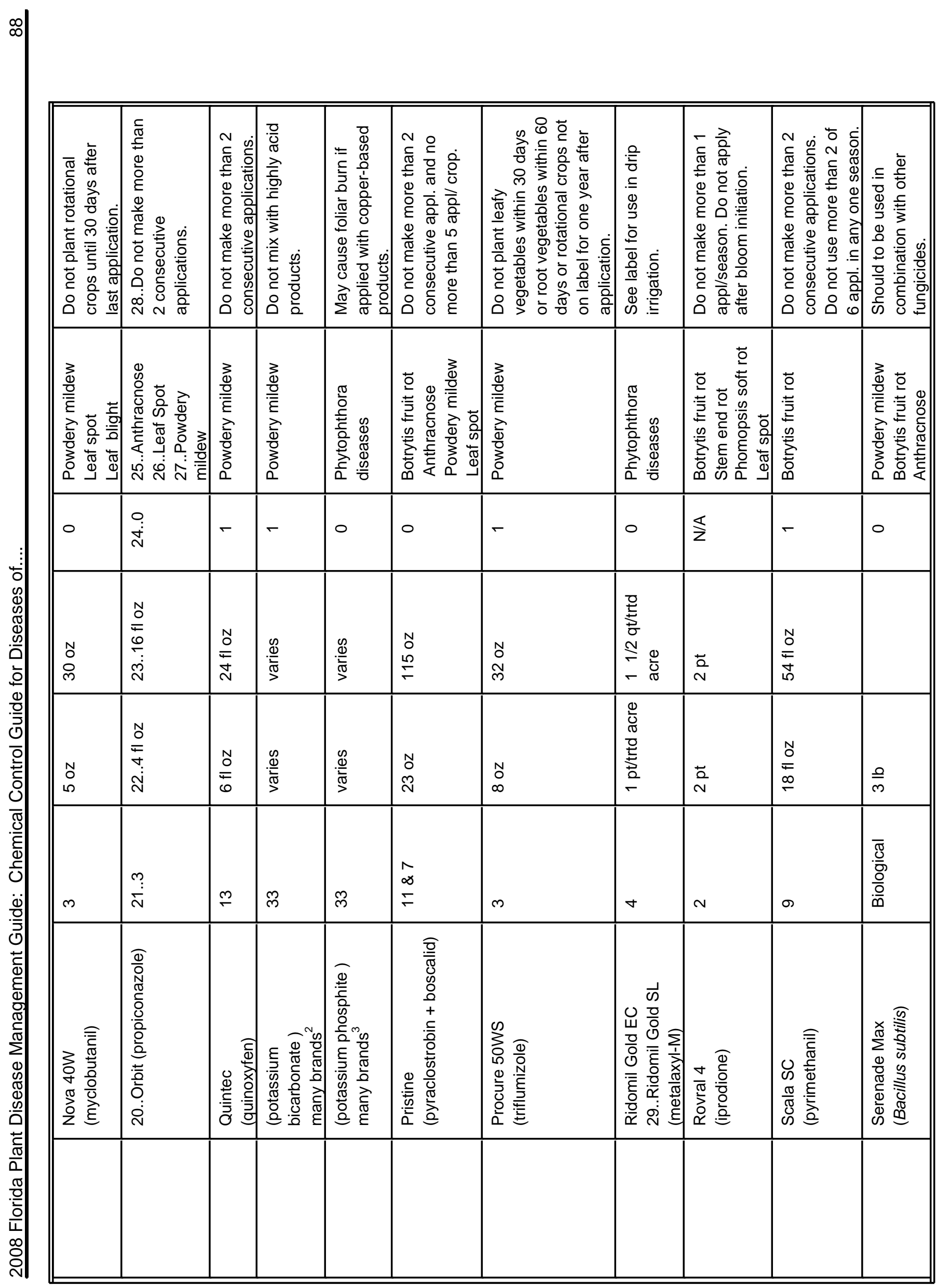




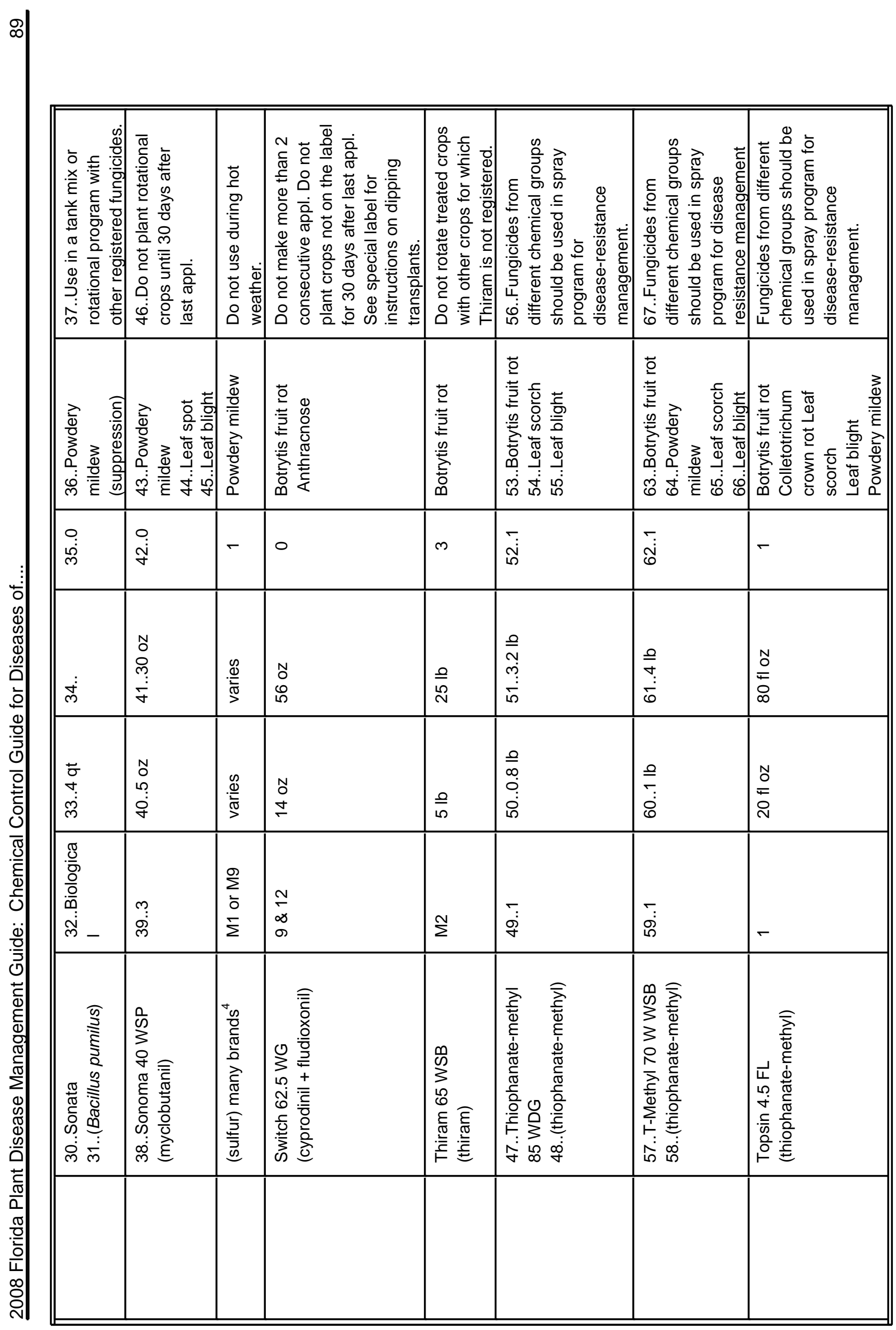




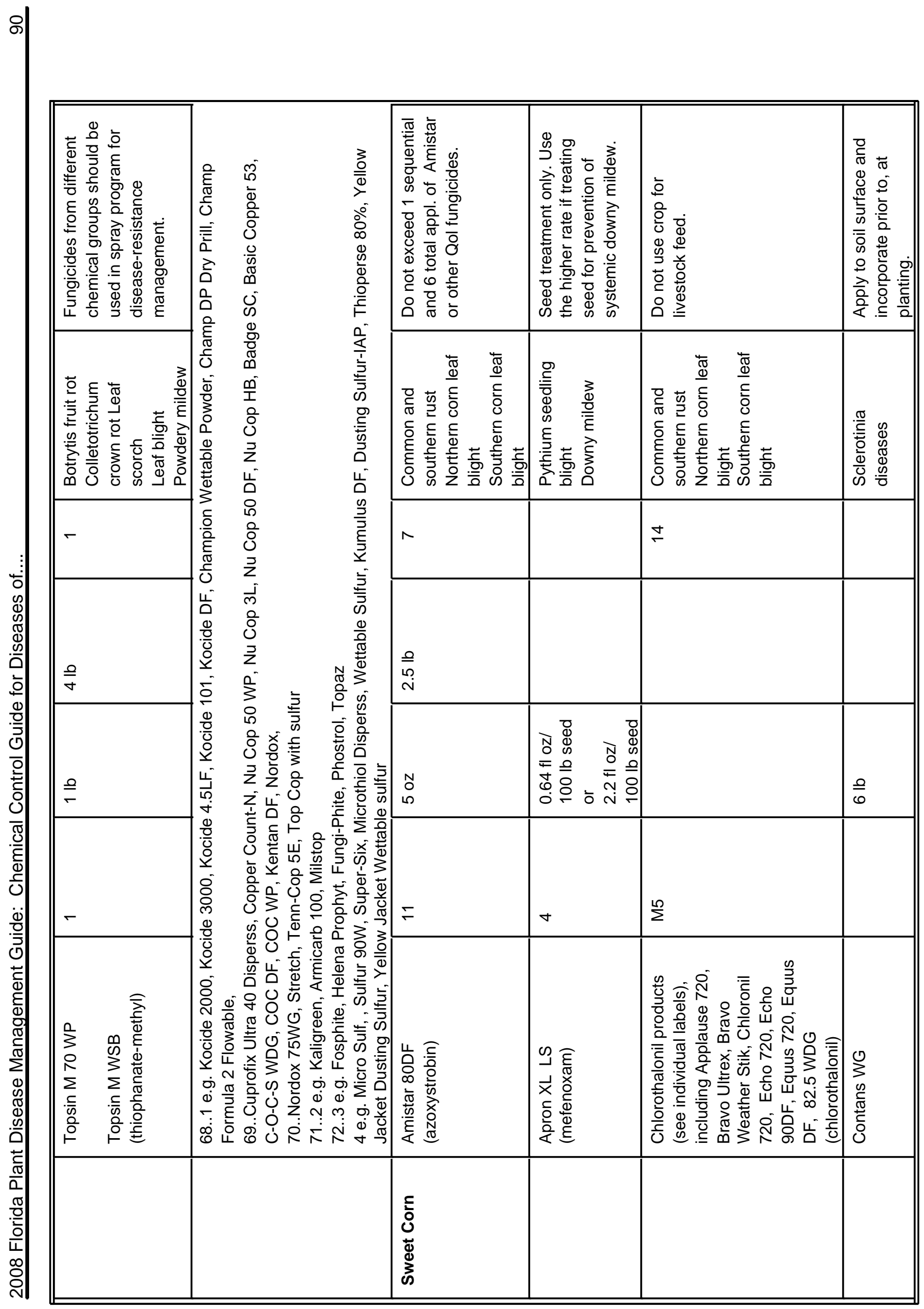




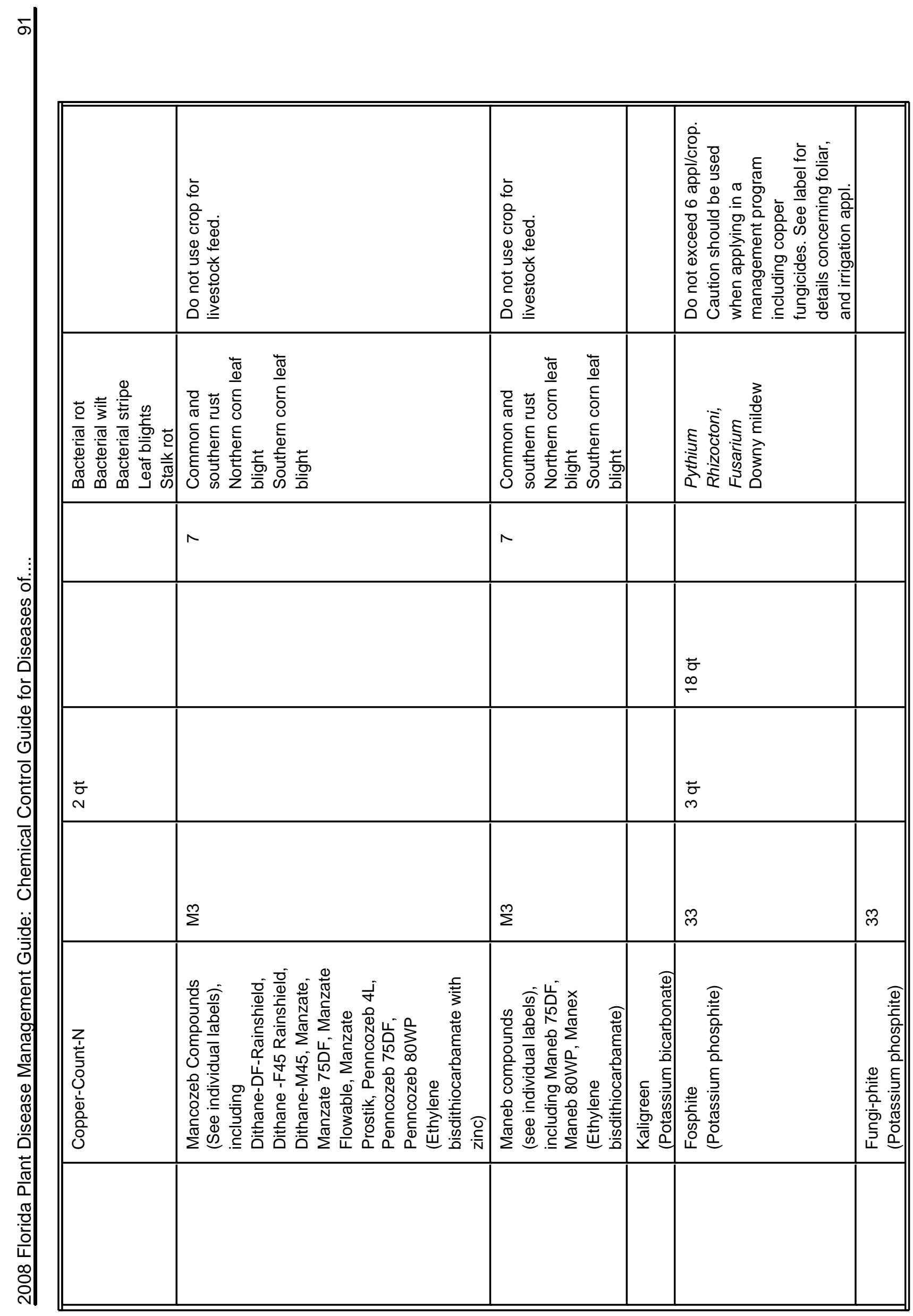




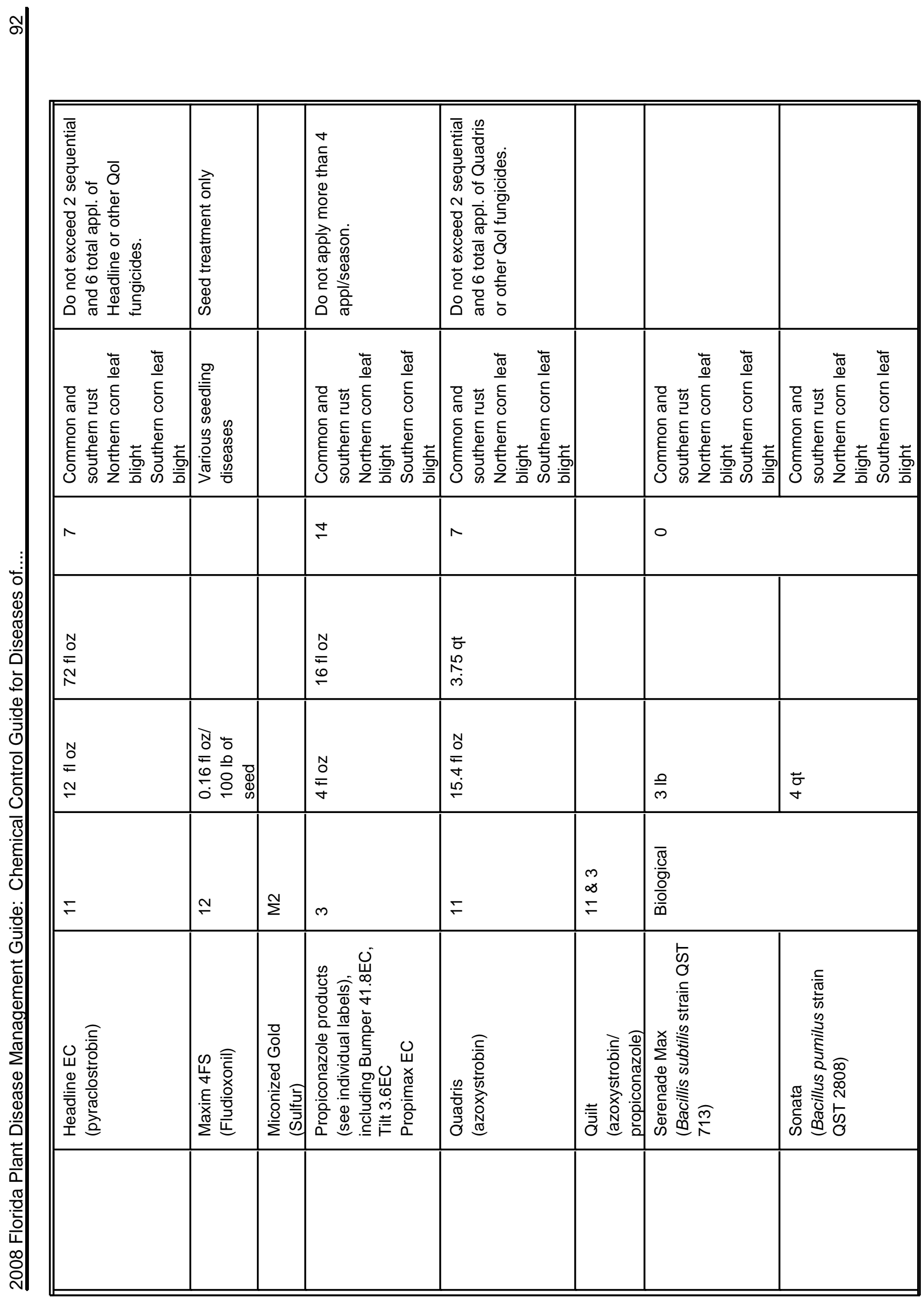




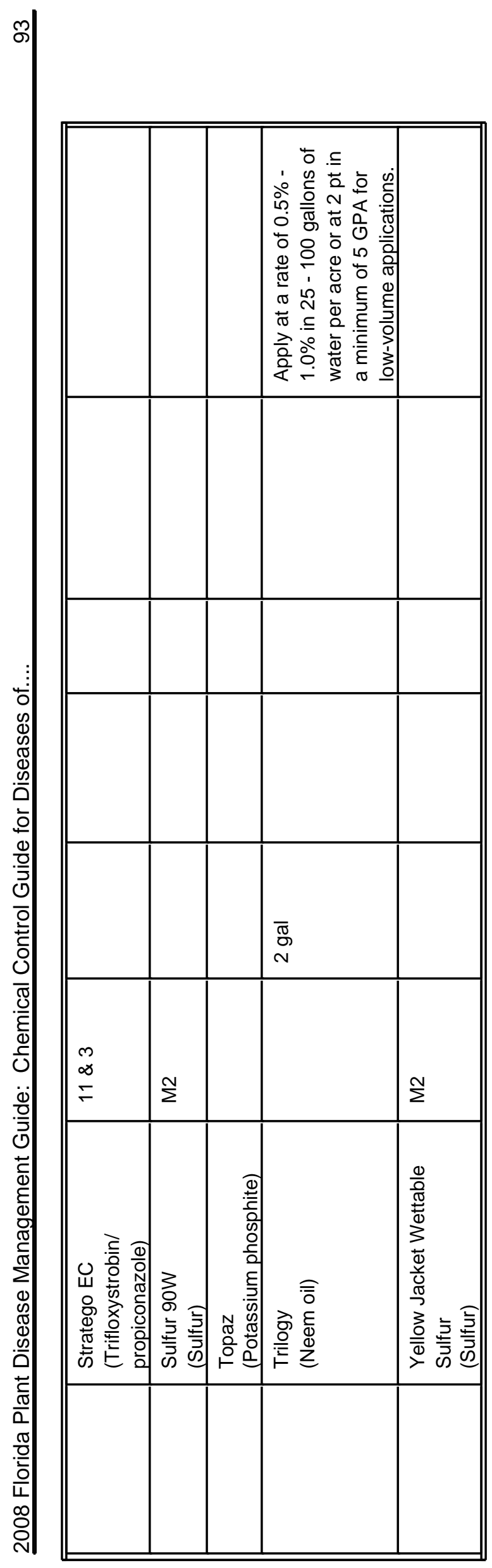

\begin{tabular}{|c|c|c|c|c|}
\hline 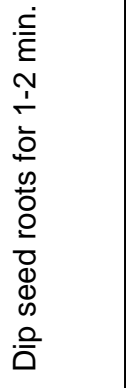 & 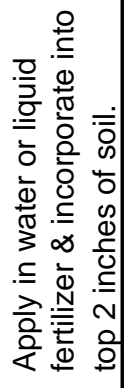 & & 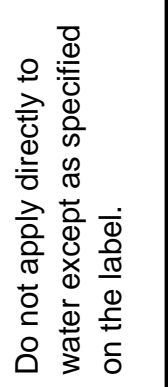 & 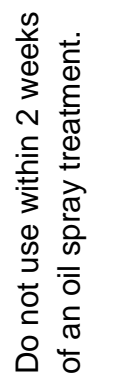 \\
\hline 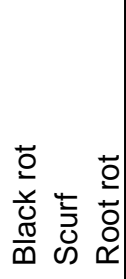 & 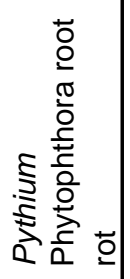 & 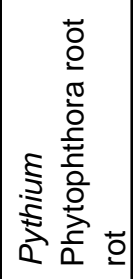 & 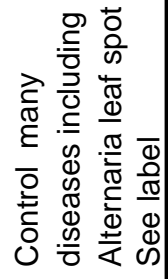 & 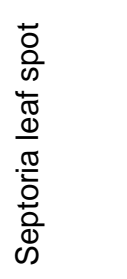 \\
\hline 0 & 0 & 0 & 0 & 0 \\
\hline & & & 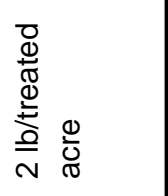 & \\
\hline 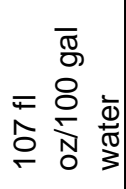 & 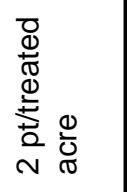 & 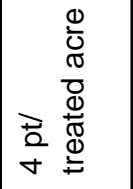 & 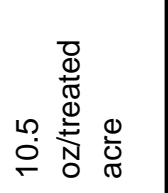 & 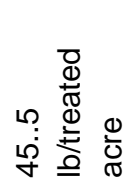 \\
\hline$\tau$ & $\nabla$ & $\nabla$ & $\mp$ & $\stackrel{N}{\Sigma}$ \\
\hline 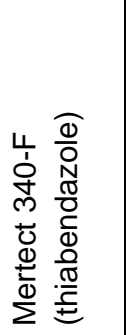 & 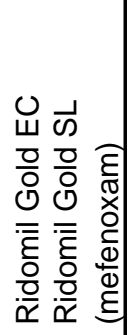 & 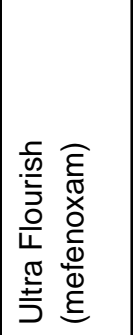 & 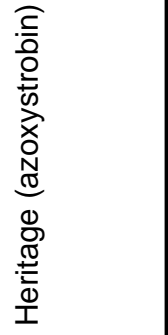 & 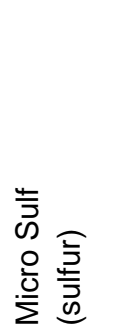 \\
\hline 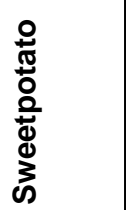 & & & & \\
\hline
\end{tabular}




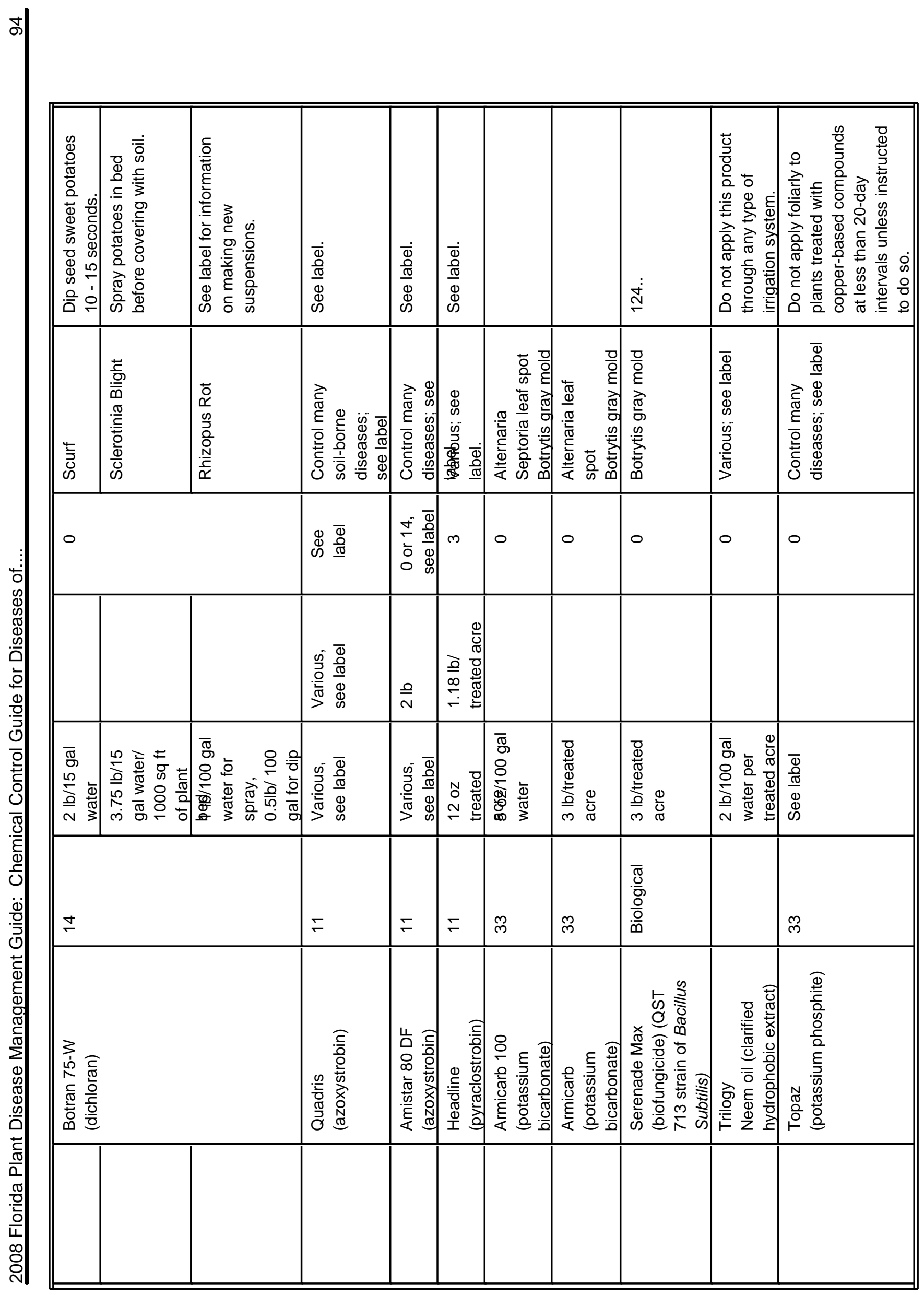




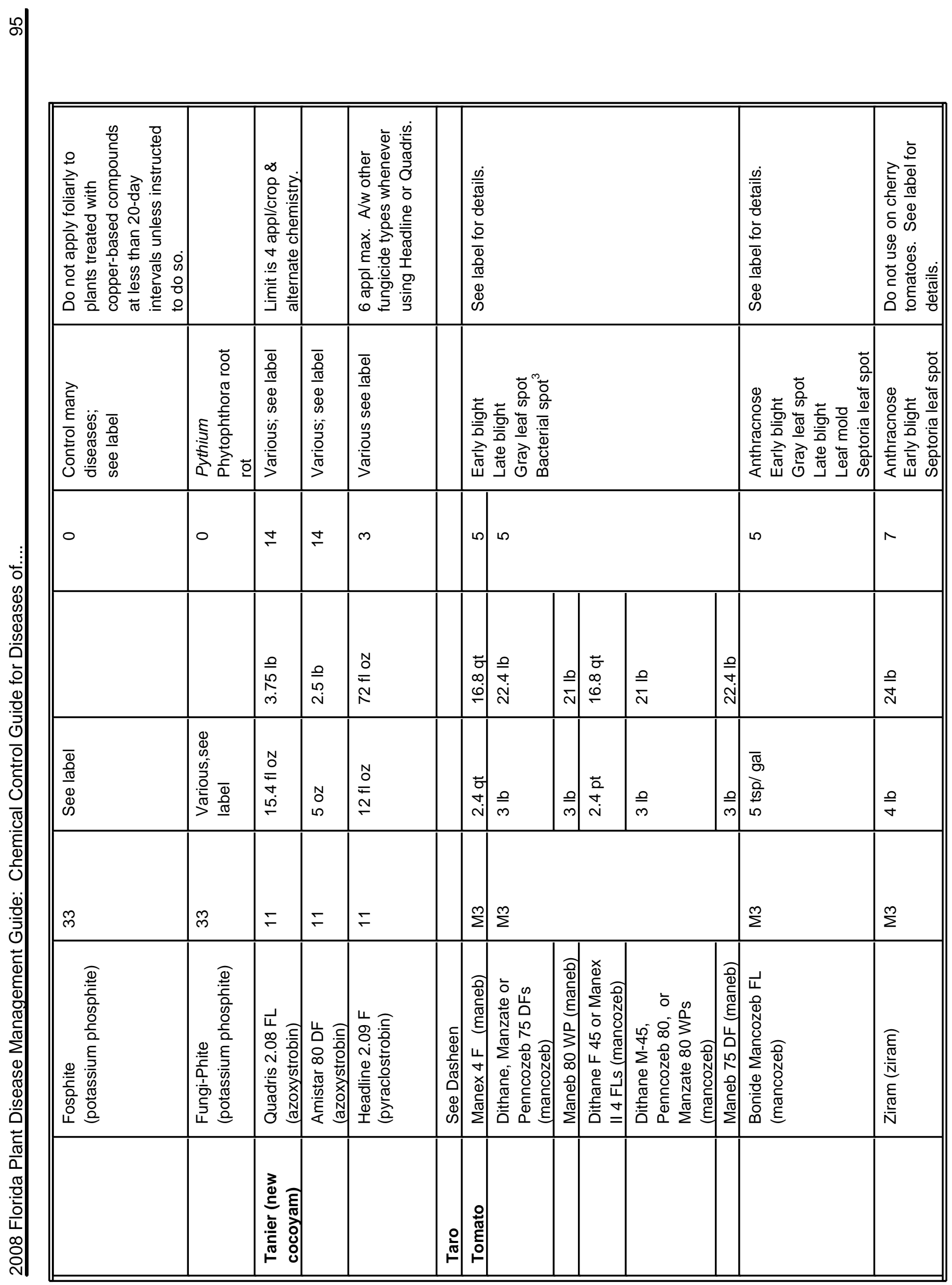




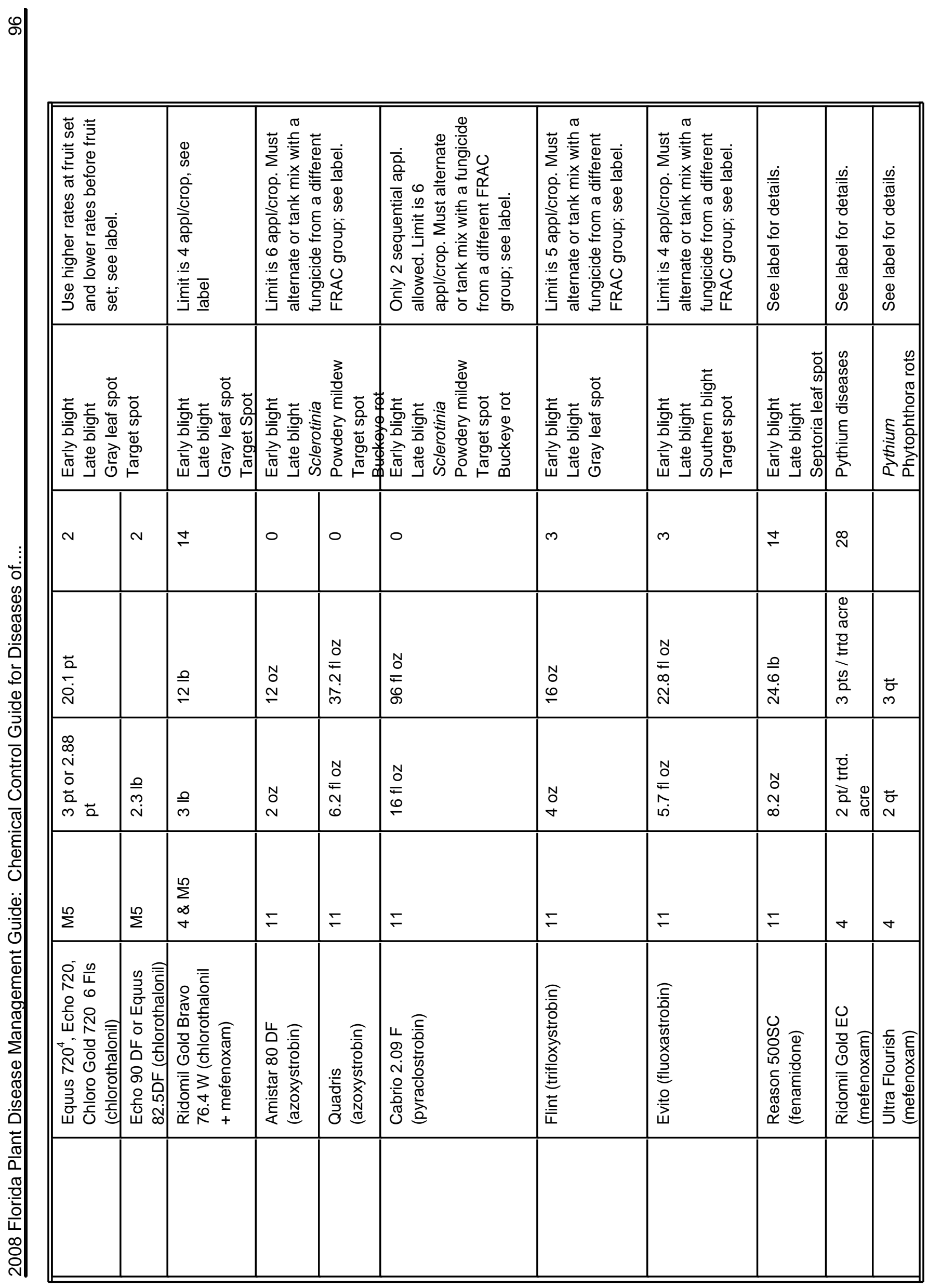




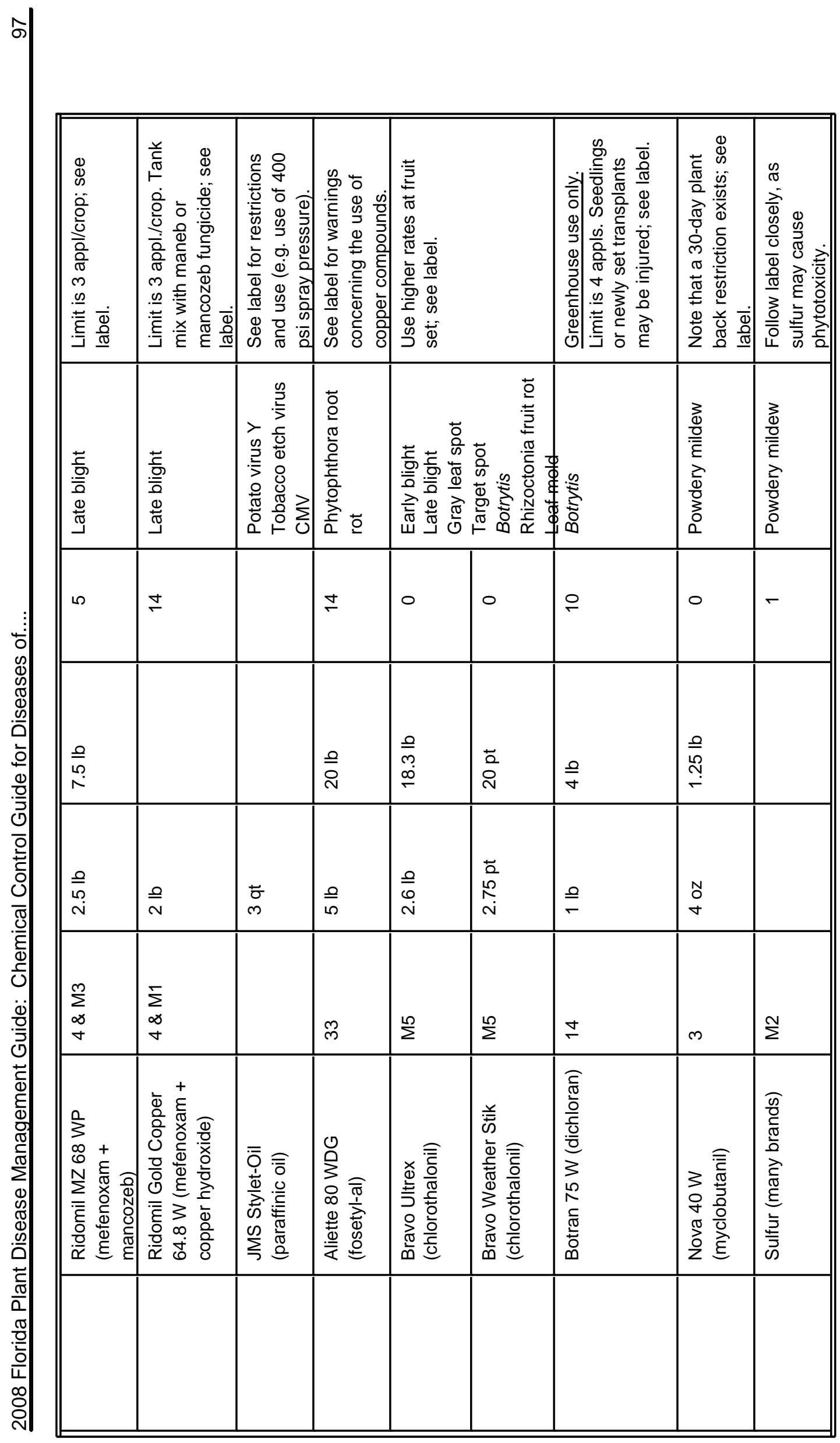




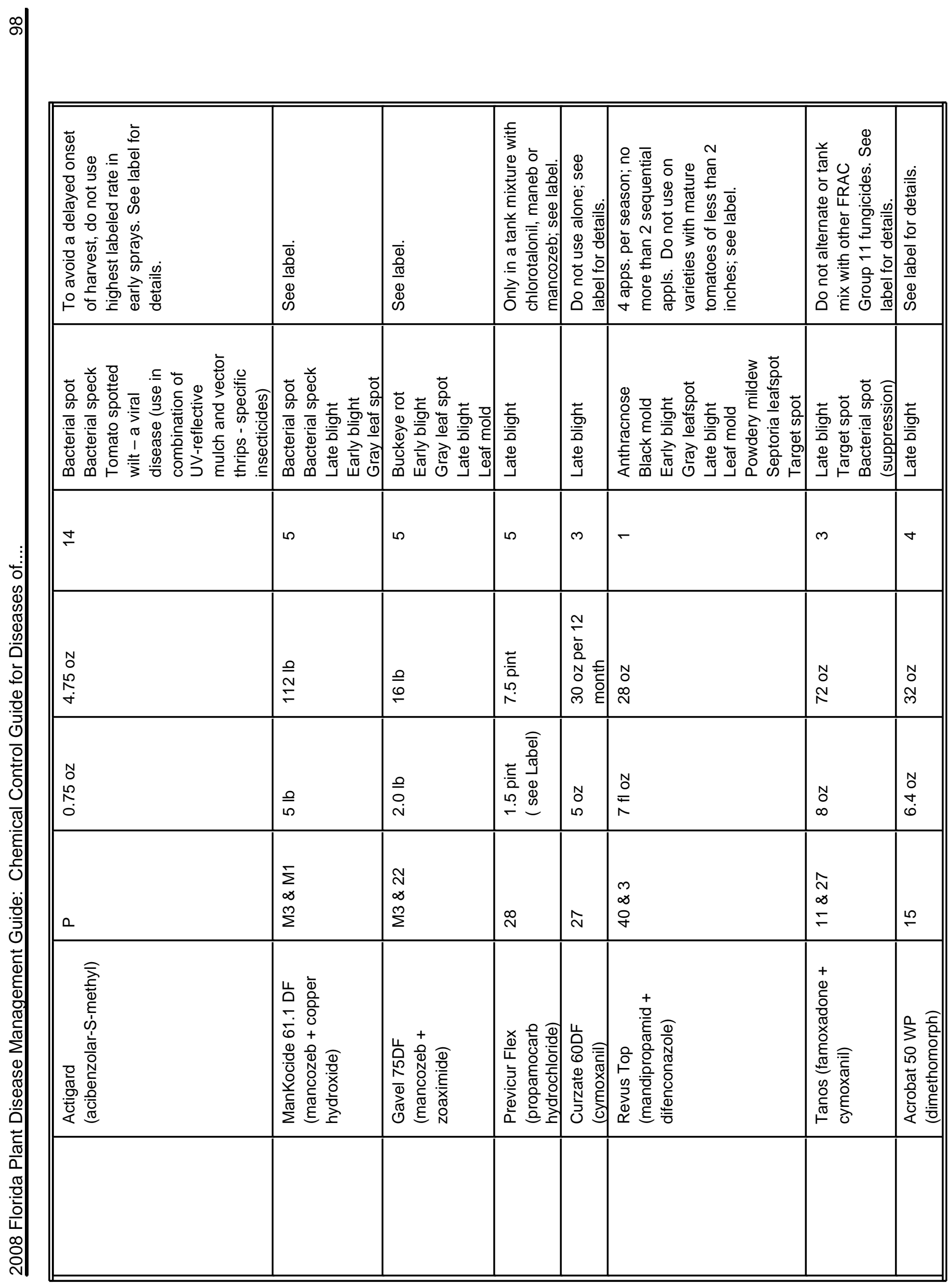




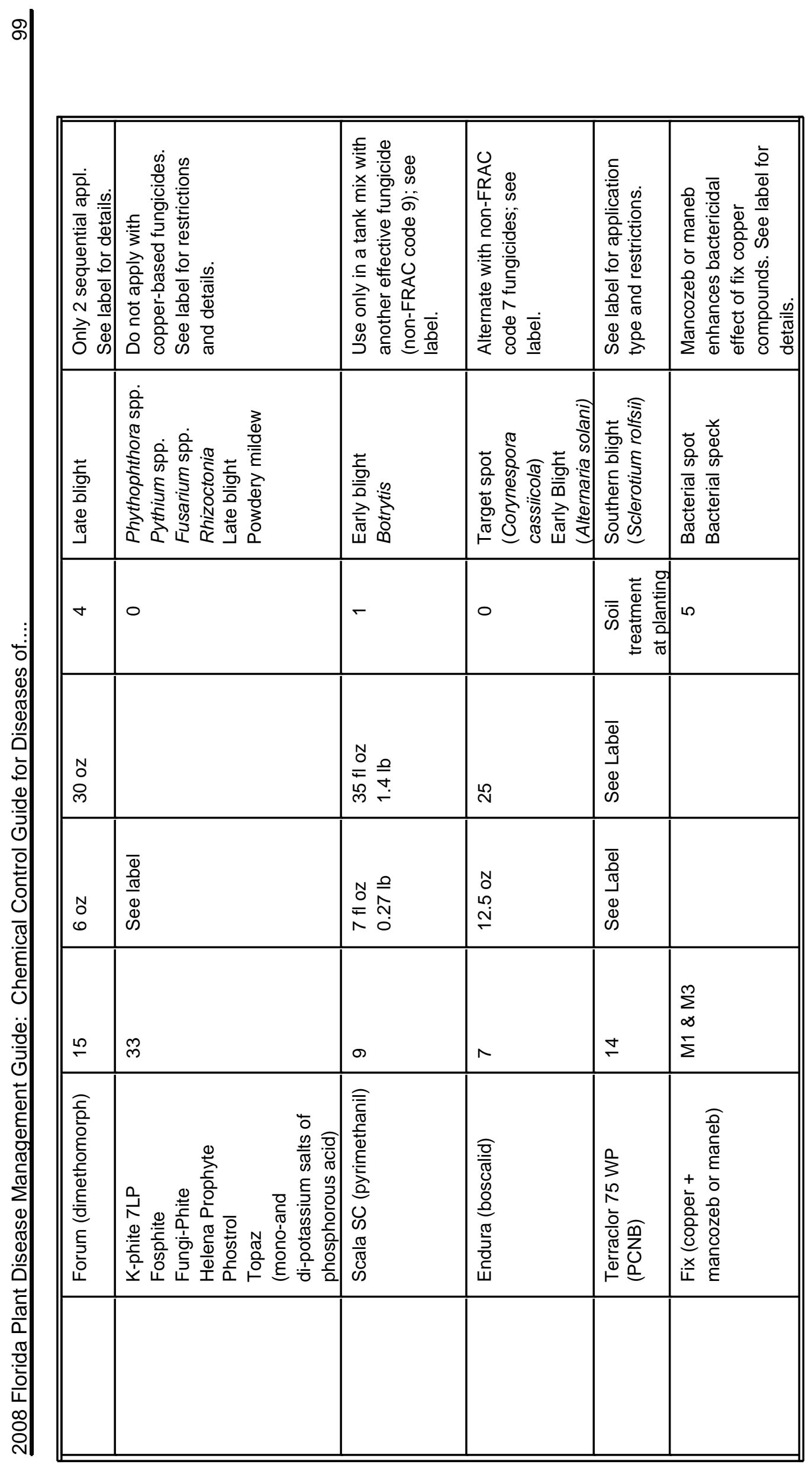




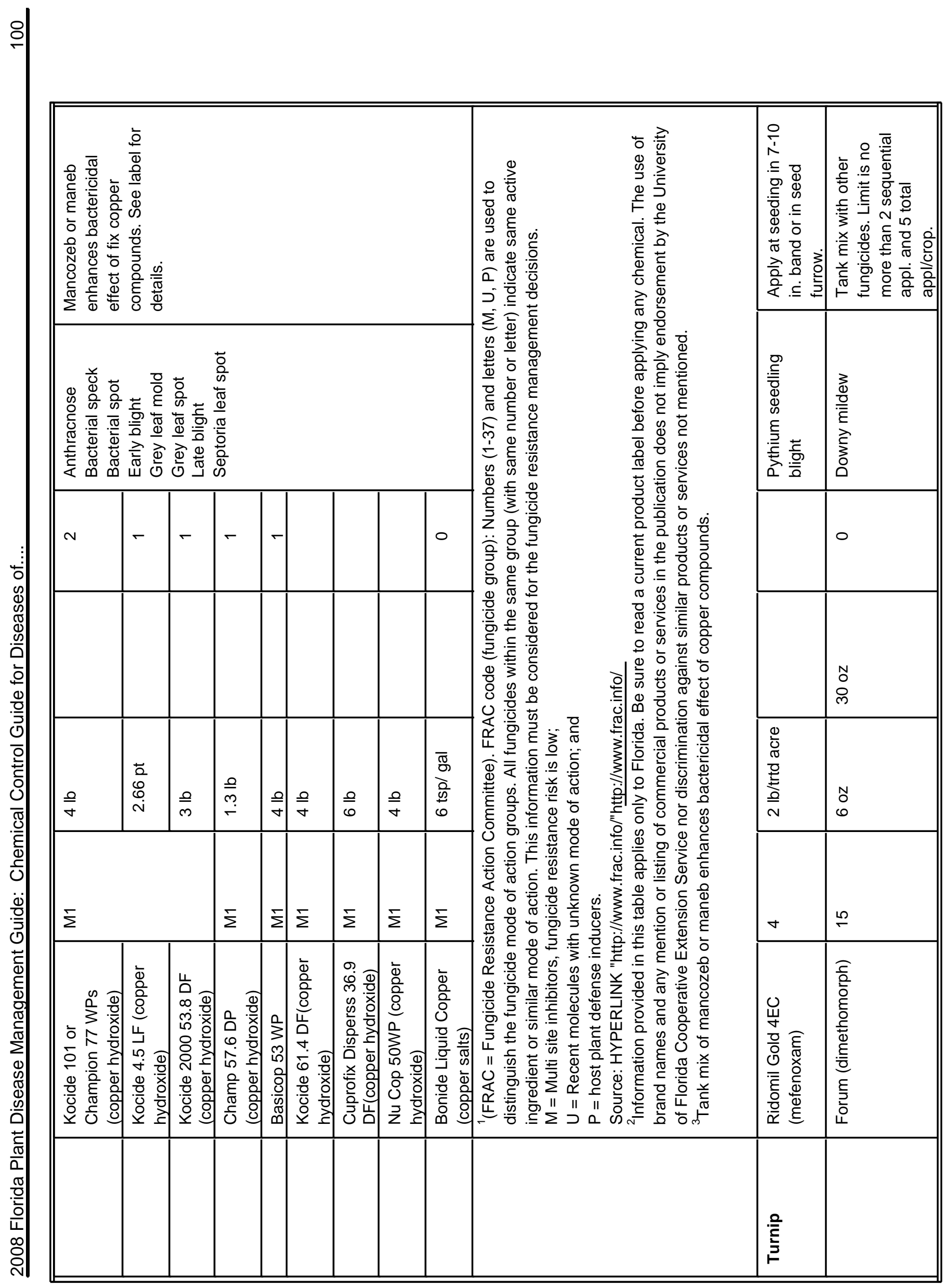




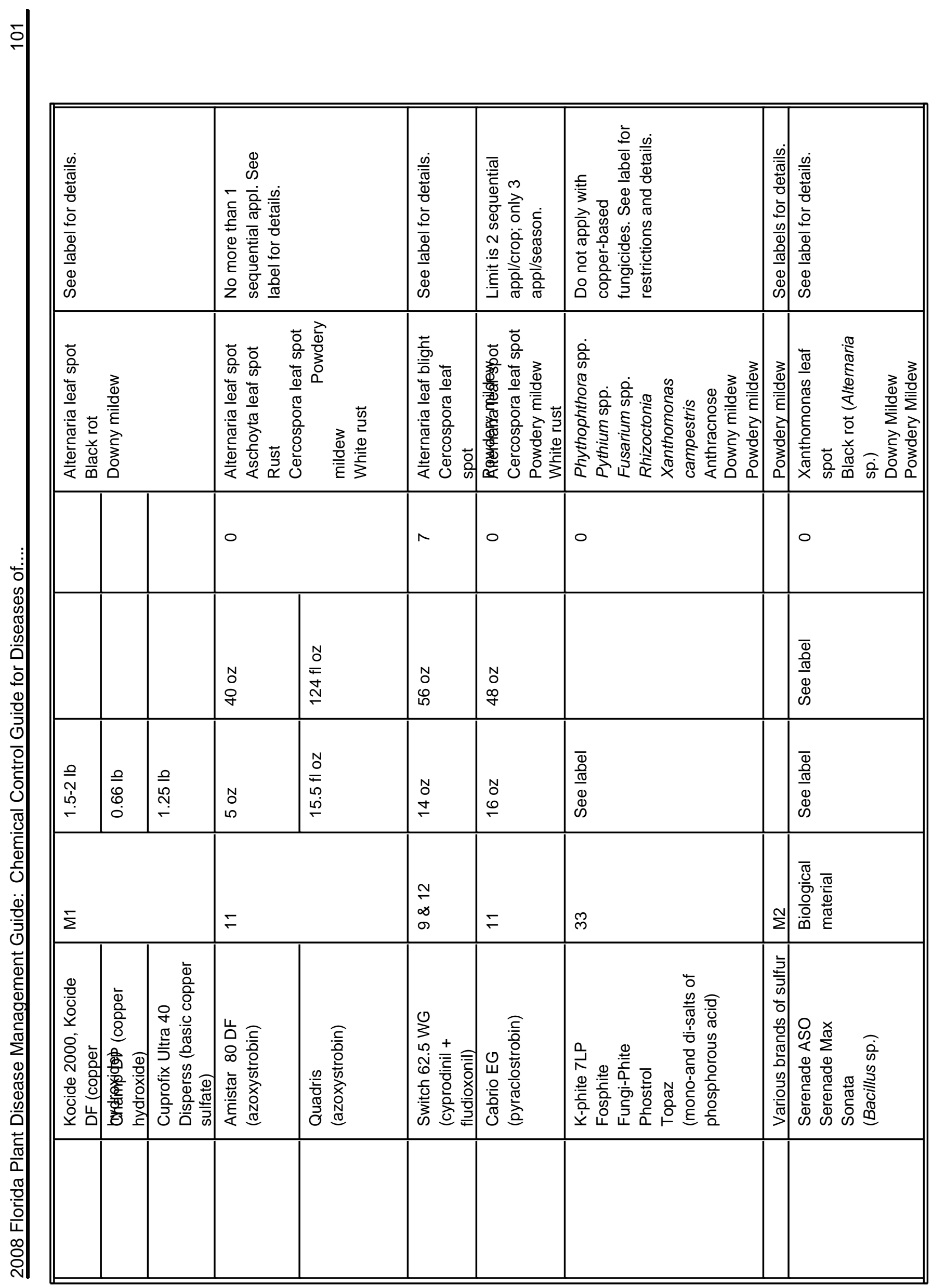




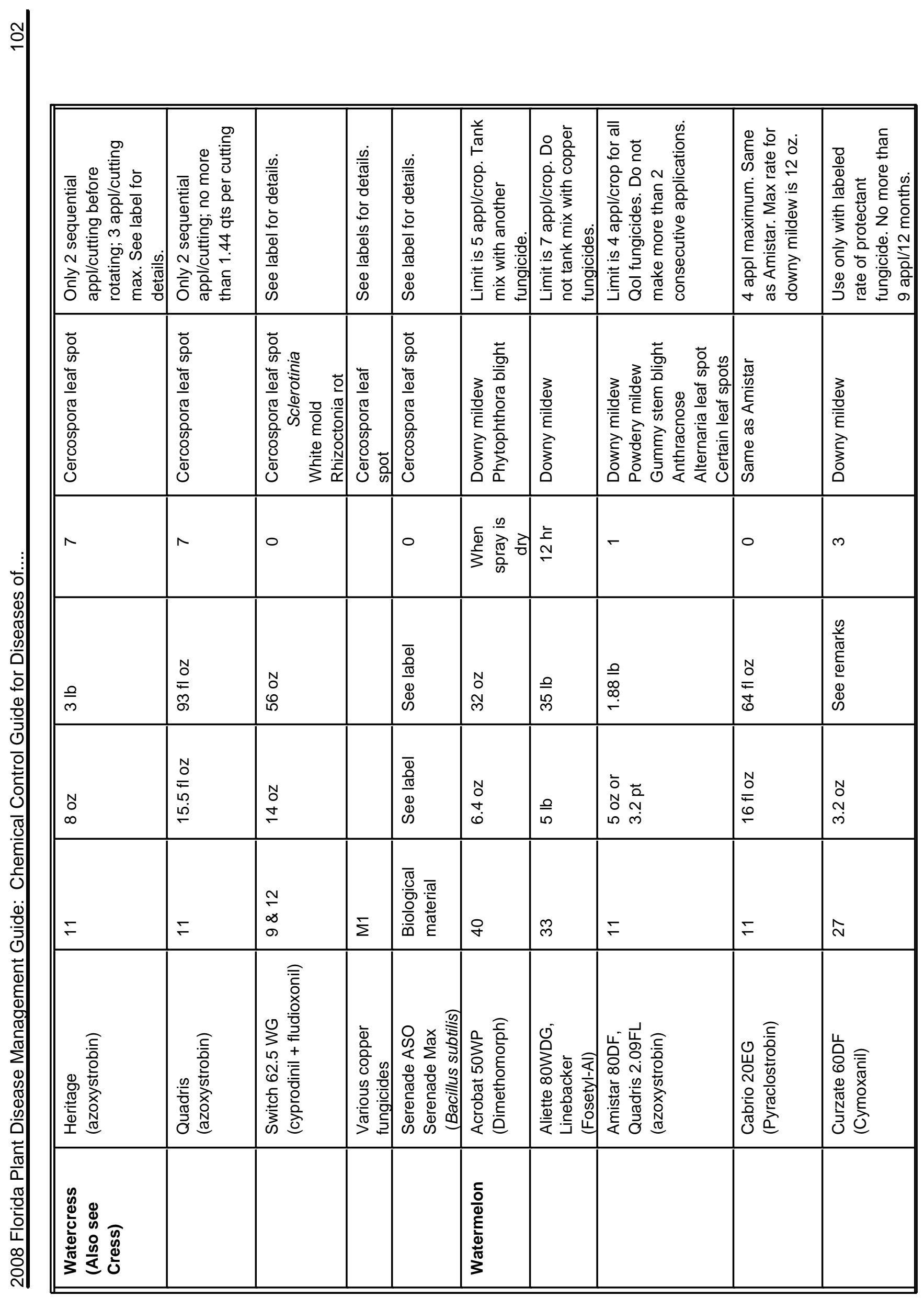




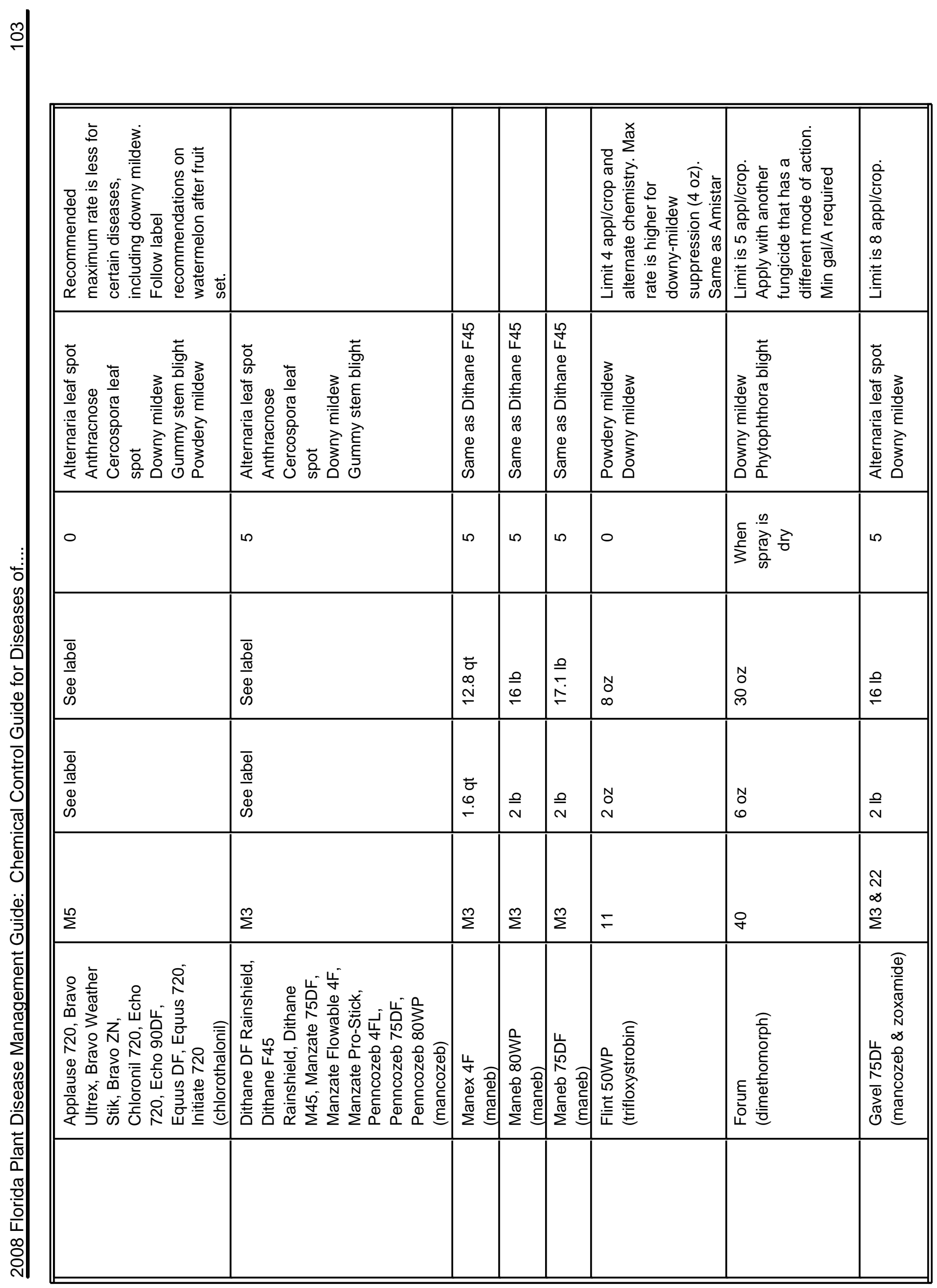




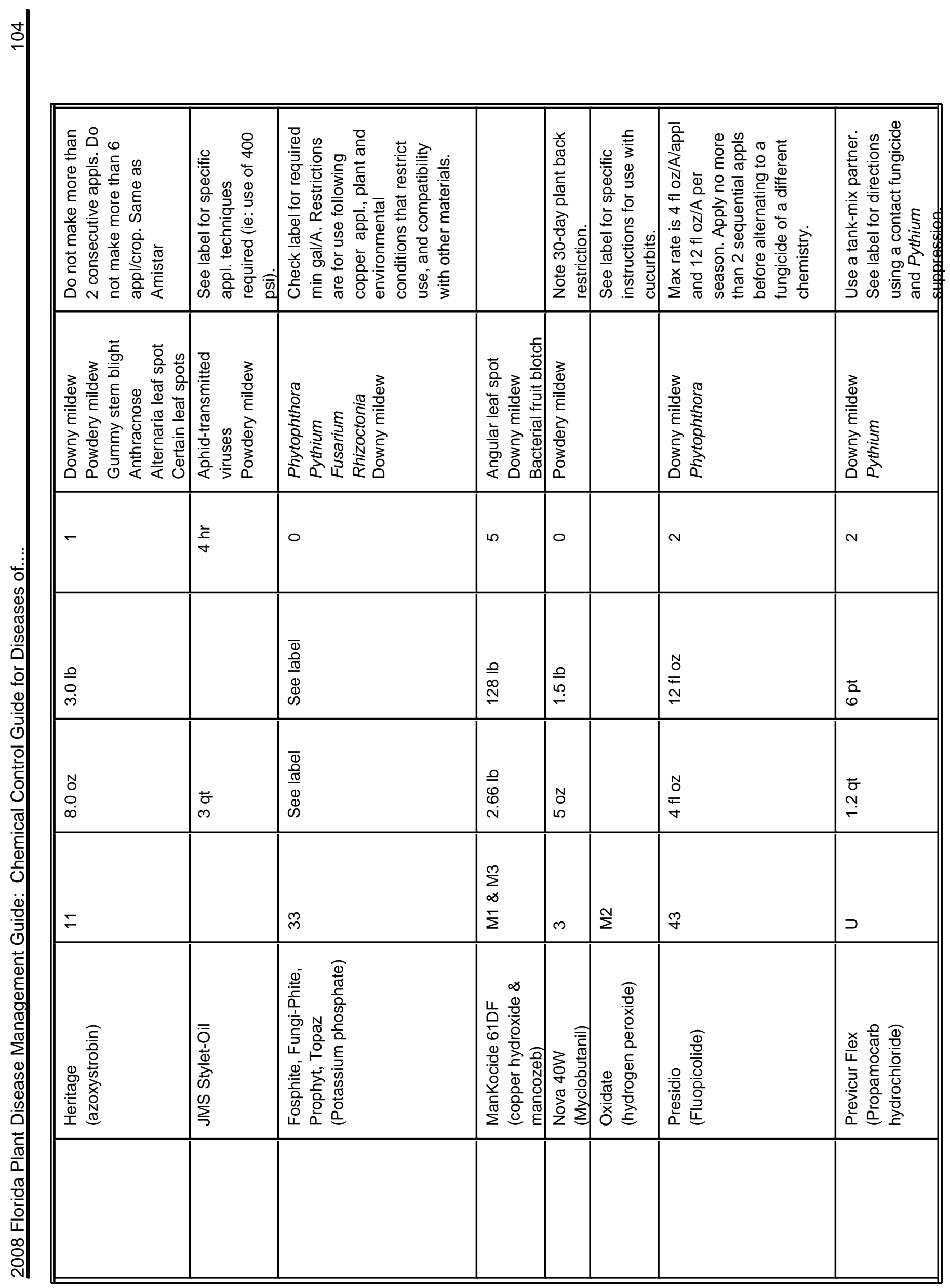




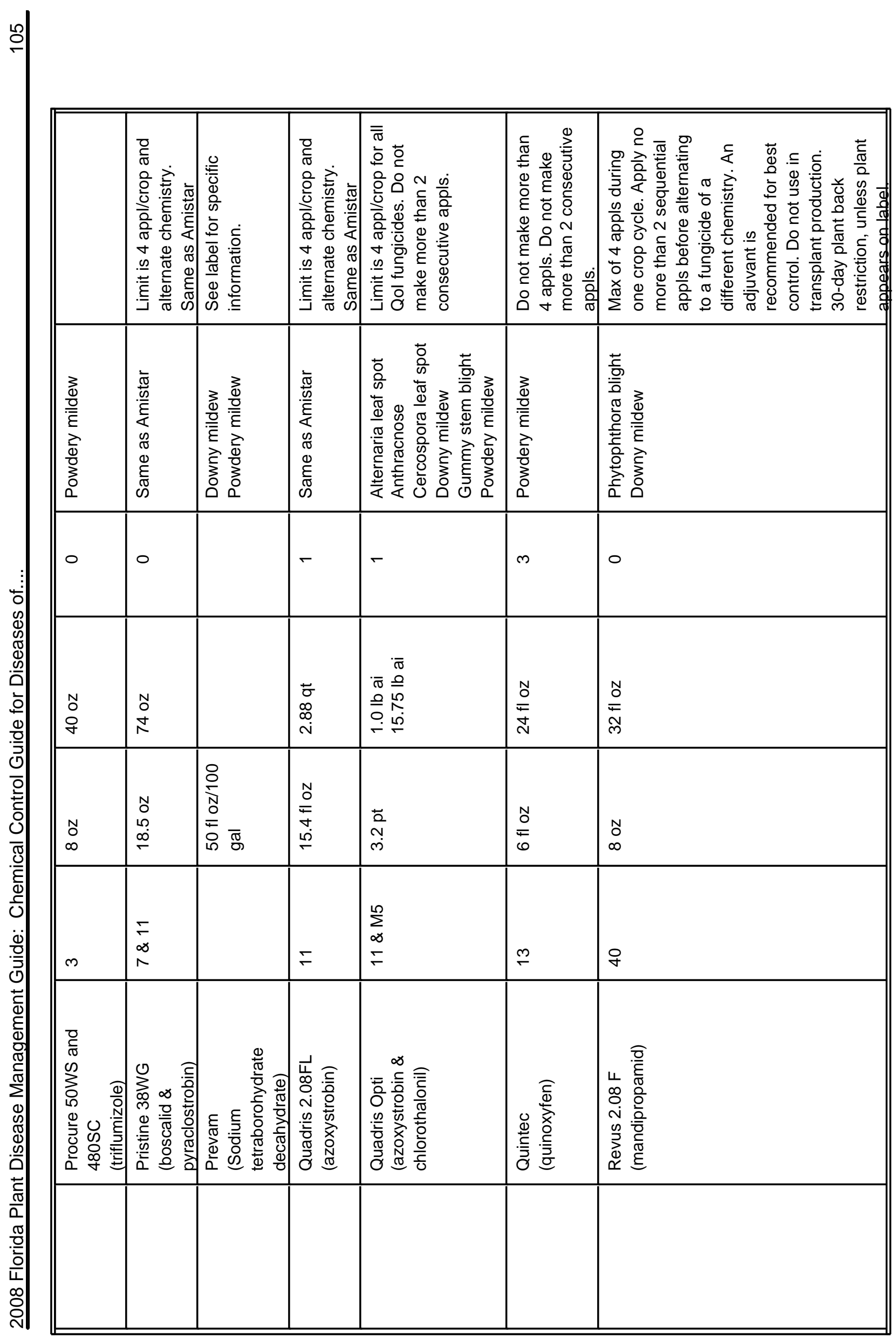




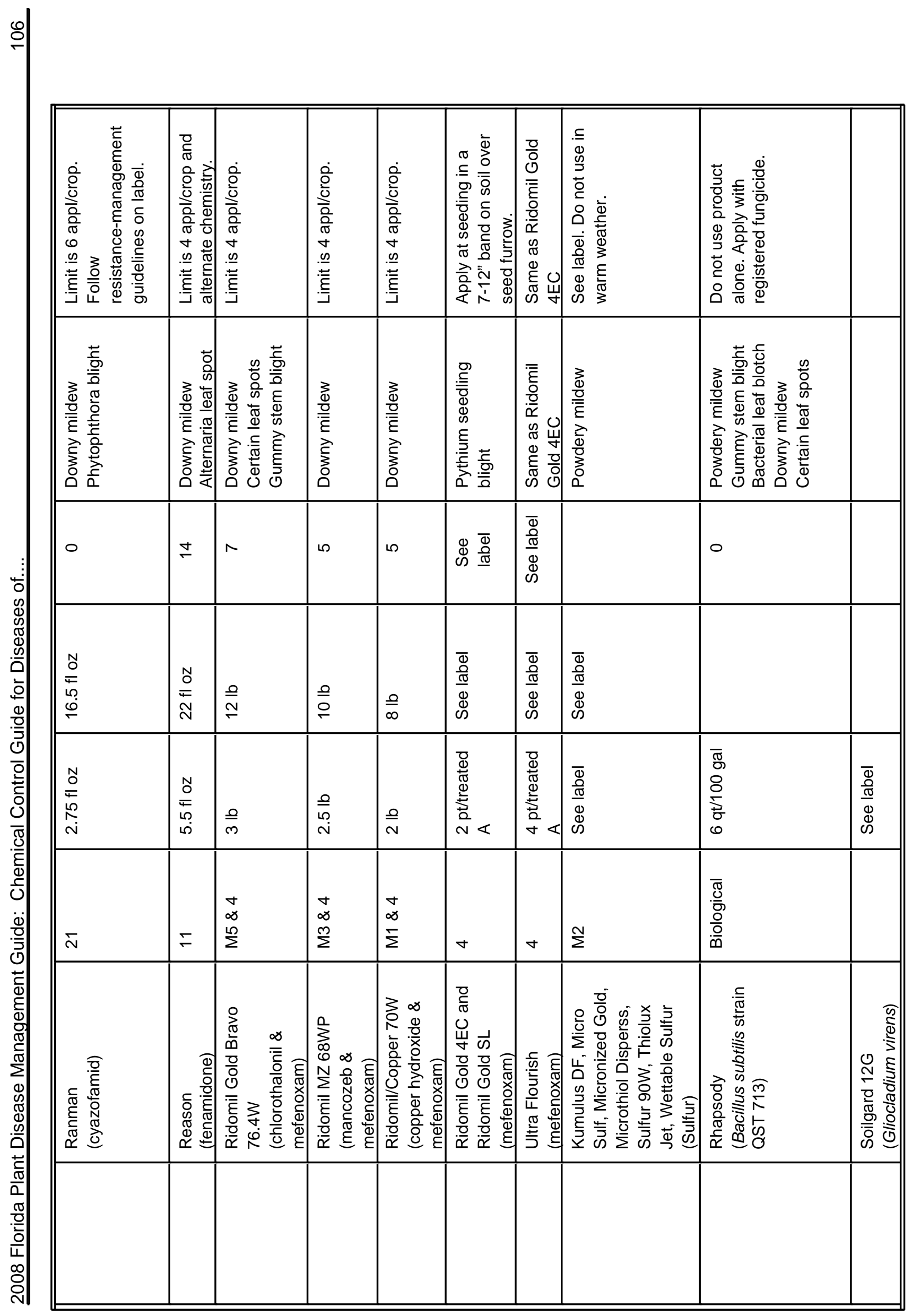




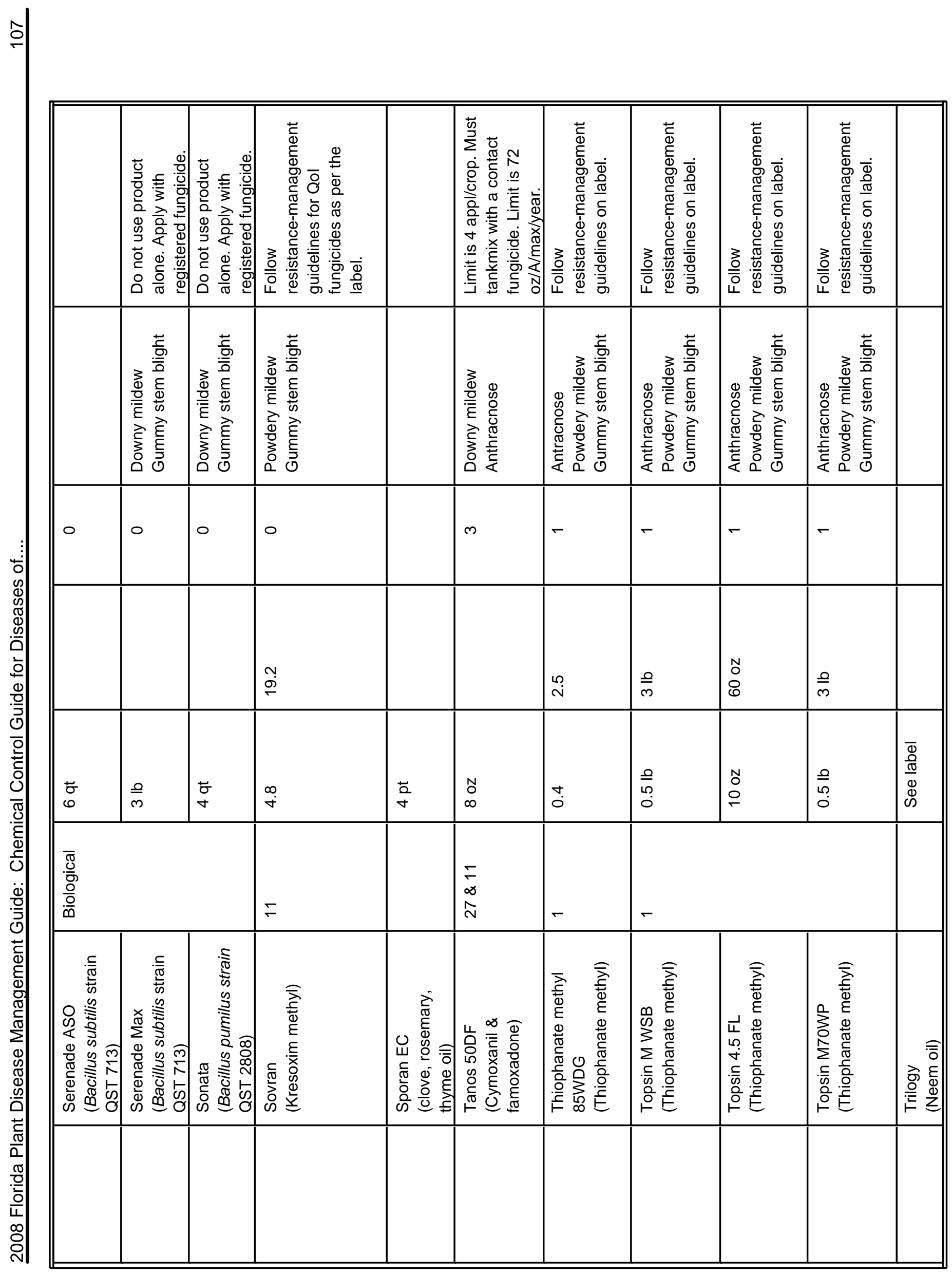




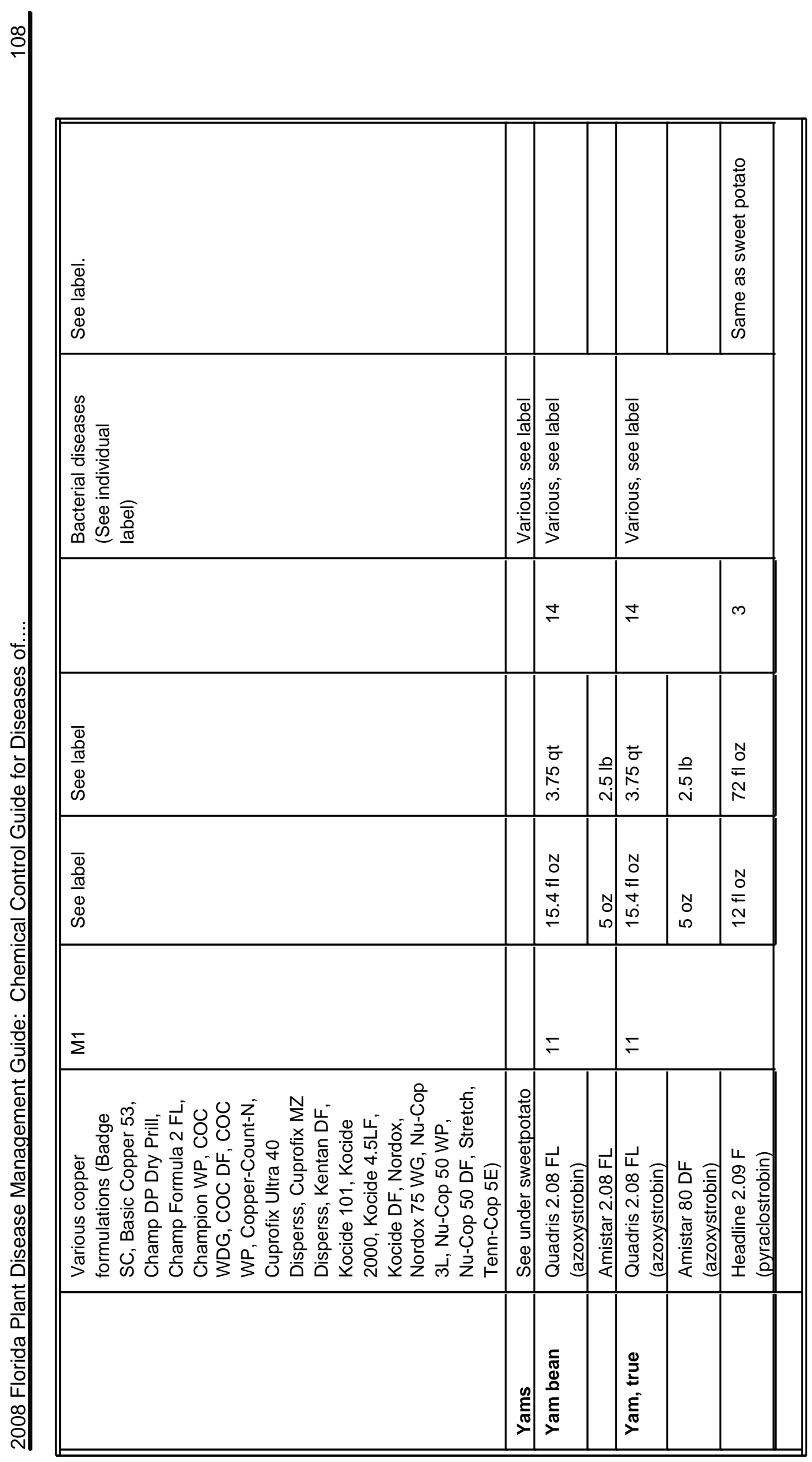

